\title{
High Maneuverability Airframe: Investigation of Fin and Canard Sizing For Optimum Maneuverability
}

by Sidra I Silton, Frank Fresconi, and Ilmars Celmins 


\section{NOTICES}

\section{Disclaimers}

The findings in this report are not to be construed as an official Department of the Army position unless so designated by other authorized documents.

Citation of manufacturer's or trade names does not constitute an official endorsement or approval of the use thereof.

Destroy this report when it is no longer needed. Do not return it to the originator. 


\section{Army Research Laboratory}

Aberdeen Proving Ground, MD 21005-5066

\section{High Maneuverability Airframe: Investigation of Fin and Canard Sizing For Optimum Maneuverability}

Sidra I Silton, Frank Fresconi, and Ilmars Celmins Weapons and Materials Research Directorate, ARL 


\section{REPORT DOCUMENTATION PAGE}

Public reporting burden for this collection of information is estimated to average 1 hour per response, including the time for reviewing instructions, searching existing data sources, gathering and maintaining the data needed, and completing and reviewing the collection information. Send comments regarding this burden estimate or any other aspect of this collection of information, including suggestions for reducing the burden, to Department of Defense, Washington Headquarters Services, Directorate for Information Operations and Reports (0704-0188), 1215 Jefferson Davis Highway, Suite 1204, Arlington, VA 22202-4302. Respondents should be aware that notwithstanding any other provision of law, no person shall be subject to any penalty for failing to comply with a collection of information if it does not display a currently valid OMB control number.

PLEASE DO NOT RETURN YOUR FORM TO THE ABOVE ADDRESS.

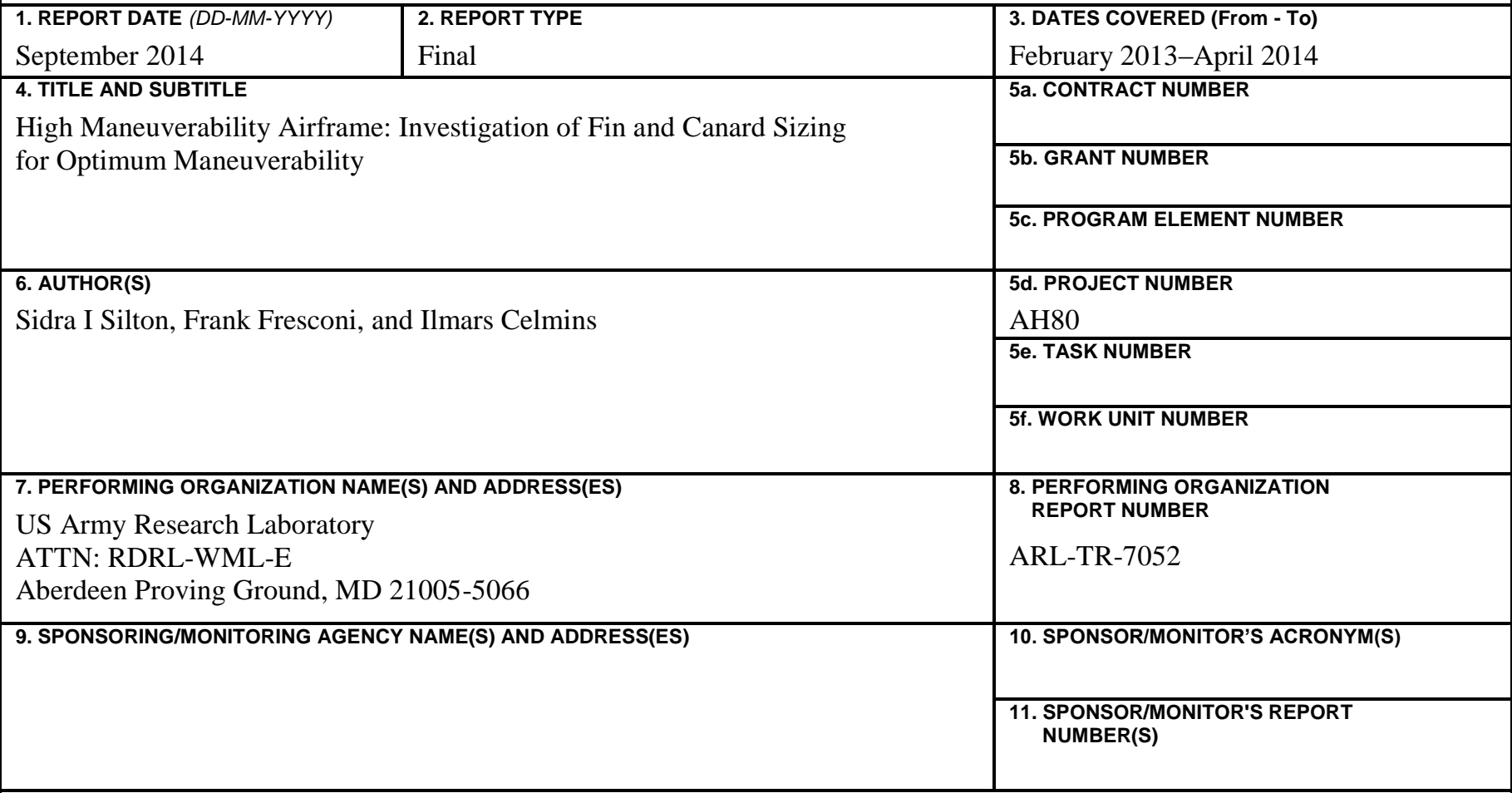

\section{DISTRIBUTION/AVAILABILITY STATEMENT}

Approved for public release; distribution is unlimited.

13. SUPPLEMENTARY NOTES

\section{ABSTRACT}

The next generation of precision munitions is likely to use a non-rolling airframe with 4 independent canards to increase maneuverability of the munition such that it can engage moving as well as stationary targets. The design of such a precision munition requires increased understanding of the airframe, of maneuver technology, and of guidance and flight control. Prior to engaging in the research on the high maneuverability airframe and maneuver system, a demonstration platform needed to be selected. The focus of this study was to design an airframe that would meet stability, range, and maneuverability requirements, while remaining simple enough so that quality research in other areas could be engaged. Advanced computational aerodynamic techniques found that the initial airframe design did not meet stability requirements as a consequence of excluding the canardfin interaction effects. Using an updated aerodynamic coefficient database, an airframe was designed using optimization parameters and realistic mass properties. An aerodynamic coefficient database and the associated flight dynamics were derived on the second configuration to enable flight simulation. Maneuvering flight simulations demonstrated that this airframe design will meet the stability, range, and maneuverability requirements.

15. SUBJECT TERMS

high maneuverability airframe, canard interaction effects

\begin{tabular}{|l|l|l|c|c|l|}
\hline \multicolumn{2}{|l|}{ 16. SECURITY CLASSIFICATION OF: } & $\begin{array}{c}\text { 17. LIMITATION } \\
\text { OF ABSTRACT }\end{array}$ & $\begin{array}{l}\text { 18. NUMBER } \\
\text { OF PAGES }\end{array}$ & $\begin{array}{l}\text { 19a. NAME OF RESPONSIBLE PERSON } \\
\text { Sidra I Silton }\end{array}$ \\
\cline { 1 - 2 } $\begin{array}{l}\text { a. REPORT } \\
\text { Unclassified }\end{array}$ & $\begin{array}{l}\text { b. ABSTRACT } \\
\text { Unclassified }\end{array}$ & $\begin{array}{l}\text { c. THIS PAGE } \\
\text { Unclassified }\end{array}$ & UU & 96 & $\begin{array}{l}\text { 19b. TELEPHONE NUMBER (Include area code) } \\
410-306-0792\end{array}$ \\
\hline
\end{tabular}




\section{Contents}

List of Figures $\quad$ v

List of Tables

$\begin{array}{ll}\text { Acknowledgments } & \text { ix }\end{array}$

1. Introduction $\quad 1$

2. Geometry 2

$\begin{array}{lr}\text { 3. Solution Techniques } & 3\end{array}$

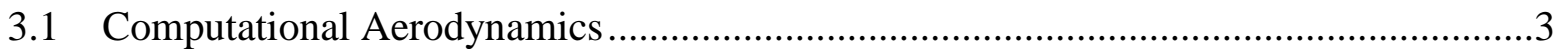

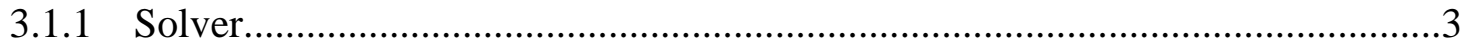

3.1.2 Numerical Grids ...................................................................................4

3.1.3 Flow Field and Boundary Conditions .....................................................

3.2 Parameter Sensitivity Technique .........................................................................

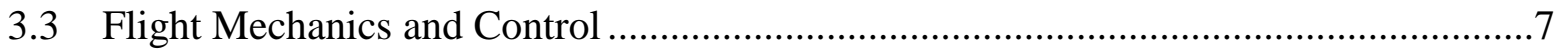

4. Results and Discussion 9

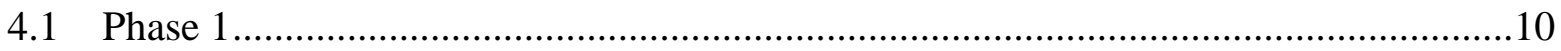

4.1.1 Static Aerodynamic Characterization ......................................................11

4.1.2 Redesign for Static Stability and Parameter Sensitivity Study .......................23

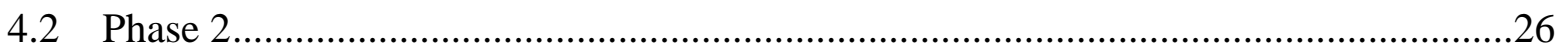

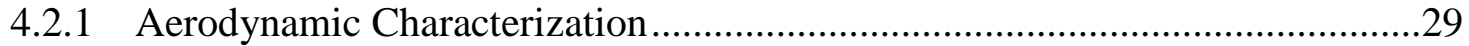

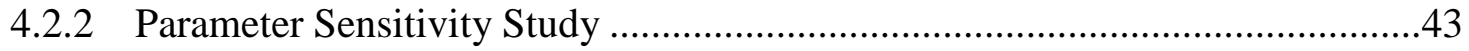

4.2.3 Linear Flight Mechanics and Control...........................................................44

4.2.4 Nonlinear Flight Mechanics and Control ......................................................45

5. Conclusions 49

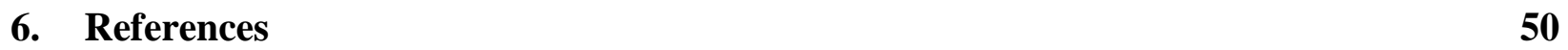

$\begin{array}{ll}\text { Appendix A. Transformations } & 53\end{array}$ 
Appendix B. Coefficient Fits for Configurations 1a and 1b

List of Symbols, Abbreviations, and Acronyms

Distribution List 


\section{List of Figures}

Fig. 1 Representative body-fin-canard geometry used during the course of this study...............2

Fig. 2 Extent of outer mesh boundary with close-up of projectile showing locations of density boxes.

Fig. 3 Initial body-fin-canard geometries Configuration 1a (top) and Configuration 1b (bottom)

Fig. 4 Canard location looking from rear of projectile

Fig. 5 Body and Fin data and fits in projectile body coordinate system for Configuration 1a, Mach 0.5

Fig. 6 CFD data and fits in for each canard in local canard coordinate system for Configuration 1a, Mach 0.5

Fig. 7 Comparison of canard coefficients in local blade coordinate and projectile body coordinates for Configuration1a, Mach 0.5

Fig. 8 Component fit build up comparison to total CFD coefficients for Configuration 1a, Mach 0.65

Fig. 9 Component fit build up comparison to total CFD coefficients for Configuration 1b, Mach 0.65

Fig. 10 Component build up of normal force and pitching moment using body-fin CFD and canard fit data from Silton and Fresconi with appropriate form factors to approximate Configuration 1b, Mach $0.65\left(f_{F}=0.75, f_{c}=1.0\right)$.

Fig. 11 Comparison of fin component as determined from component buildup with form factors and CFD simulation for Configuration 1b, Mach 0.65 .

Fig. 12 Comparison of body component as determined from component buildup with form factors and CFD simulation for Configuration 1b, Mach 0.65 .

Fig. 13 Comparison of total canard component as determined from component buildup with form factors and CFD simulation for Configuration 1b, Mach 0.65

Fig. 14 Component fit build up of normal force and pitching moment with $4^{\circ}$ canard deflection for Configuration 1a, Mach 0.65.

Fig. 15 Parameter sensitivity study using Configuration 1 a aerodynamic data with $f_{F}=1.95$, $f_{c}=0.75$, and $\delta=4^{\circ}$

Fig. 16 Parameter sensitivity study using aerodynamic data from Configuration $1 \mathrm{~b}$ with $f_{F}=$ 1.7, $f_{c}=0.75$, and $\delta=4^{\circ}$

Fig. 17 Projectile components used to calculate mass properties ..........................................27

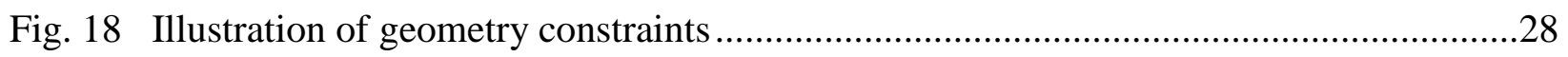

Fig. 19 Third body-fin-canard geometry (Configuration 2) ...............................................28

Fig. 20 Body and fin component fits for Configuration 2, Mach 0.65 ...................................31 
Fig. 21 Canard component fits in local blade coordinate system for Configuration 2, Mach 0.65

Fig. 22 Normal force, pitching moment, and center-of-pressure location build ups using CFD component fits with $0^{\circ}$ canard deflection for Configuration 2, Mach 0.65 .35

Fig. 23 Component build up of normal force and pitching moment using scaled component contributions $\left(f_{F}=2.0, f_{c}=0.7, x_{c}=0.88, X_{c g}=2.63\right)$ from CFD analysis of Configuration 1a to obtain theoretical values for Configuration 2, Mach 0.65 .

Fig. 24 Comparison of body component for Configuration 2 as determined from component buildup using Configuration 1a aerodynamics with form factors and CFD simulations, Mach 0.65

Fig. 25 Comparison of fin component for Configuration 2 as determined from component buildup using Configuration 1a aerodynamics with form factors and CFD simulations, Mach 0.65

Fig. 26 Comparison of canard component for Configuration 2 as determined from component buildup using Configuration 1a aerodynamics with form factors and CFD simulations, Mach 0.65

Fig. 27 Normal force, pitching moment, and center-of-pressure location build ups using CFD component fits with $4^{\circ}$ canard deflection for Configuration 2, Mach 0.65

Fig. 28 Normal force, pitching moment, and center-of-pressure location build ups using CFD component fits with $6^{\circ}$ canard deflection for Configuration 2, Mach 0.65

Fig. 29 Pitch plane Mach number contours with pressure coefficient contours on fins and canards $\left(\delta=0^{\circ}\right)$ with streamlines at various angles of attack for Configuration 2, Mach 0.65

Fig. 30 Individual canard streamlines, rear view, for Configuration 2, Mach 0.65.

Fig. 31 Optimization analysis using Configuration 2 aerodynamic data with $f_{F}=0.8, f_{c}=$ 1.5 , and $\delta=4^{\circ}$

Fig. 32 Response and commands in pitch from linear modeling and simulation

Fig. 33 Trajectory and aerodynamic angle histories for representative flight of

Configuration 2

Fig. 34 Controlled response histories in roll, pitch, and yaw for representative flight of Configuration 2

Fig. 35 Canard deflection histories for representative flight of Configuration 2......................48

Fig. A-1 CFD coordinate system and aerodynamic angles...............................................54

Fig. A-2 Body-fixed coordinate system and aerodynamic angles ......................................55

Fig. A-3 Definition of canard orientation when looking from the rear of the projectile relative to body coordinates

Fig. A-4 Definition of ballistic coefficients and aerodynamic angles in the body coordinate system .......

Fig. B-1 Body and fin component fits for Configuration 1a, Mach 0.5 
Fig. B-2 Individual canard fits in local canard coordinate system for Configuration 1a, Mach 0.5

Fig. B-3 Component fit build up comparison to total CFD coefficients for Configuration 1a, Mach 0.5

Fig. B-4 Body and fin component fits for Configuration 1a, Mach 0.65

Fig. B-5 Individual canard fits in local canard coordinate system for Configuration 1a, Mach 0.65

Fig. B-6 Component fit build up comparison to total CFD coefficients for Configuration 1a, Mach 0.65 .65

Fig. B-7 Body and fin component fits for Configuration 1a, Mach 0.8 .66

Fig. B-8 Individual canard fits in local canard coordinate system for Configuration 1a, Mach 0.8

Fig. B-9 Component fit build up comparison to total CFD coefficients for Configuration 1a, Mach 0.8 .68

Fig. B-10 Body and fin component fits for Configuration 1b, Mach 0.5

Fig. B-11 Individual canard fits in local canard coordinate system for Configuration 1b, Mach 0.5

Fig. B-12 Component fit build up comparison to total CFD coefficients for Configuration 1b, Mach 0.5 .71

Fig. B-13 Body and fin component fits for Configuration 1b, Mach 0.65

Fig. B-14 Individual canard fits in local canard coordinate system for Configuration 1b, Mach 0.65

Fig. B-15 Component fit build up comparison to total CFD coefficients for Configuration 1b, Mach 0.65 .74

Fig. B-16 Body and fin component fits for Configuration 1b, Mach 0.8 .75

Fig. B-17 Individual canard fits in local canard coordinate system for Configuration 1b, Mach 0.8 .76

Fig. B-18 Component fit build up comparison to total CFD coefficients for Configuration 1b, Mach 0.8 


\section{List of Tables}

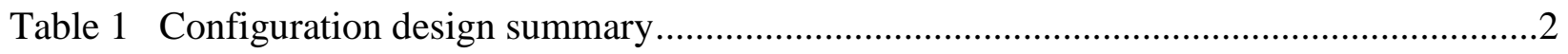

Table 2 Body component of aerodynamic coefficient fits for Configurations $1 \mathrm{a}$ and $1 \mathrm{~b}$............15

Table 3 Total fin component of aerodynamic coefficient fits for Configurations $1 \mathrm{a}$ and $1 \mathrm{~b}$.......16

Table 4 Aerodynamic coefficient fits Canard 1for Configurations 1a and 1b............................16

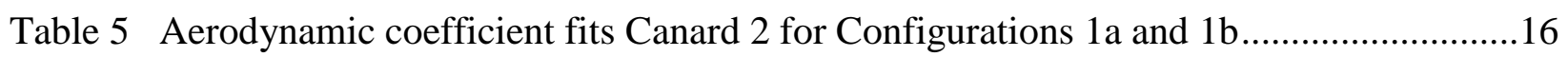

Table 6 Aerodynamic coefficient fits Canard 3 for Configurations $1 \mathrm{a}$ and $1 \mathrm{~b}$...........................17

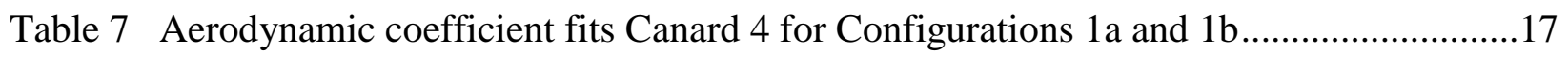

Table 8 Static aerodynamic coefficient fits for Configuraion 2 at Mach 0.65, body and fin

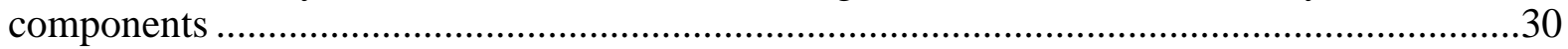

Table 9 Static aerodynamic coefficient fits for Canard 1 for Configuration 2, Mach 0.65 ..........34

Table 10 Static aerodynamic coefficient fits for Canard 2 for Configuration, Mach 0.65 ...........34

Table 11 Static aerodynamic coefficient fits for Canard 3 for Configuration 2, Mach 0.65 ........34

Table 12 Static aerodynamic coefficient fits for Canard 4 for Configuration 2, Mach 0.65 ........34 


\section{Acknowledgments}

The author would like to thank the following personnel:

- Dr James DeSpirito, Army Research Laboratory (ARL), Flight Sciences Branch, for multiple conversations relating to the computational fluid dynamics simulations.

- Mrs Karen Heavey, ARL, Flight Sciences Branch, for performing the technical review.

- The US Department of Defense (DOD) High-Performance Computing Modernization Program (HPCMP) at the ARL DOD Supercomputing Resource Center (ARL DSRC), Aberdeen Proving Ground, MD, for the grant of high-performance computing time. 
INTENTIONALLY LEFT BLANK. 


\section{Introduction}

Precision munitions research is currently an area of focus throughout the United States Armed Services. Within the Army, rolling munitions using movable lifting surfaces for maneuver control have been and are continuing to be developed. ${ }^{1,2}$ However, the maneuverability of such munitions is limited to the munition being positioned in the proper orientation to move in the desired direction. Research shows ${ }^{3}$ that a non-rolling airframe using 4 independent canards in a skid-to-turn maneuver is the most effective method by which to guide a munition with movable lifting surfaces. As the armed services' desire to engage moving targets as well as stationary targets increases, the need for understanding the aerodynamics associated with highly maneuverable airframes also increases.

To that end, the 2013 US Army Research Laboratory (ARL) Low Cost Hyper Accurate Weapon (LCHAW) mission program seeks to develop a highly maneuverable projectile that is able to acquire, track, and intercept a moving target as the natural extension of the Very Affordable Precision Projectile (VAPP) program that was successfully able to guide-to-hit a stationary target. This effort requires research in a few key areas: high maneuverability airframe, maneuver technology, guidance and flight control, and image-based navigation, all at low cost.

Prior to engaging in the research on the high maneuverability airframe and maneuver system, a demonstration platform needed to be selected. Choosing a demonstration platform for the moving target mission program required a projectile configuration that would meet stability, range, and maneuverability requirements for the demonstration, while remaining simple enough so that quality research could be engaged. Initially, the $83-\mathrm{mm}$ shoulder-launched munition under development at the Armament Research, Development, and Engineering Center (ARDEC), Picatinny Aresenal, NJ, was chosen. However, previous work ${ }^{4}$ found that that munition would not meet the requirements of the current demonstration platform. Still, it was decided that this 83-mm shoulder-launched munition would serve as the starting point for the design because it had many positive characteristics.

The front end of the shoulder-launched munition was satisfactory from a flight perspective and provided enough volume for guidance sensors and electronics. However, the rear end of the munition did not meet the aerodynamic requirements. Therefore, it was decided that the rear end of the shoulder-launched munition would be modified to that of a standard, artillery-like boattail. ${ }^{4}$ While the previous study ${ }^{4}$ included optimization of both the fins and canards, the interaction effects between them were not considered. Previous research has shown that these interaction effects are critical to the performance of the projectile because the downwash of the canard vortices can reduce the effectiveness of the fins, which could have detrimental effects. ${ }^{2,5-7}$ 
This report describes the computational fluid dynamics (CFD) simulations and follow-on analyses completed to optimize the entire munition's configuration, including the maneuver system and interaction effects. The resulting configuration will be used as the demonstration platform for the remainder of the research under the LCHAW mission program.

\section{Geometry}

Three body-tail-canard configurations were investigated during the course of this study: Configuration 1a, Configuration 1b, and Configuration 2. All aerodynamic coefficients were determined for simplified geometries; all gaps and slots in the body that would be needed to accommodate the fins and canards were not included. Figure 1 shows a representative body-tailcanard configuration. Each geometric model was created in SolidWorks ${ }^{8}$ and has a body reference diameter of $83 \mathrm{~mm}$ ( 1 caliber). The body geometry, which did not vary among configurations, consisted of a hemispherical nose cap; a nearly cylindrical body $320.38 \mathrm{~mm}$ (3.86 cal.) long; and a $66.4 \mathrm{~mm}(0.8 \mathrm{cal})$ long, $7^{\circ}$ boattail. The overall length was $427.45 \mathrm{~mm}(5.15$ cal.). During the course of the study, the projectile center-of-gravity $\left(X_{c g}\right)$, the number of fins, the fin span, chord, and geometry, and the canard dither axis location $\left(x_{c}\right)$ and span (but not chord) were varied between the configurations. A summary of the 3 configuration designs can be found in Table 1. Specifics of each configuration are discussed in Section 4.

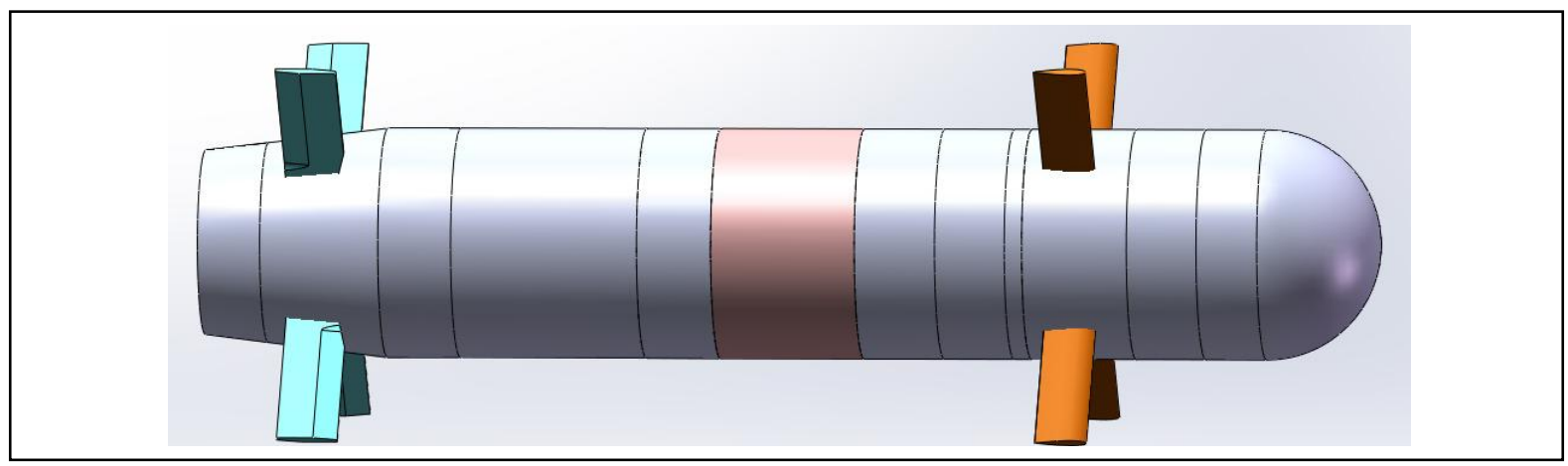

Fig. 1 Representative body-fin-canard geometry used during the course of this study

Table 1 Configuration design summary

\begin{tabular}{|c|c|c|c|c|c|c|c|c|}
\hline Configuration & $\begin{array}{l}\boldsymbol{X}_{\boldsymbol{c g}}(\mathbf{m m} \\
\text { From } \\
\text { Nose) }\end{array}$ & $\begin{array}{c}\text { No. } \\
\text { of Fins }\end{array}$ & $\begin{array}{c}\text { Fin Span } \\
(\mathbf{m m})\end{array}$ & $\begin{array}{c}\text { Fin } \\
\text { Chord } \\
(\mathbf{m m})\end{array}$ & Fin Geometry & $\begin{array}{l}\boldsymbol{x}_{\boldsymbol{c}}(\mathbf{m m} \\
\text { Forward } \\
\left.\text { of } \boldsymbol{X}_{\boldsymbol{c g}}\right)\end{array}$ & $\begin{array}{c}\text { Canard } \\
\text { Span } \\
(\mathbf{m m})\end{array}$ & $\begin{array}{c}\text { Canard } \\
\text { Chord } \\
(\mathbf{m m})\end{array}$ \\
\hline 1a & 200 & 4 & 188.4 & 22.0 & double bevel & 68.89 & 240.48 & 18.86 \\
\hline 1b & 200 & 4 & 188.4 & 22.0 & double bevel & 91.30 & 192.72 & 18.86 \\
\hline 2 & 218 & 8 & 199.9 & 19.05 & bent fin tip & 73.79 & 192.72 & 18.86 \\
\hline
\end{tabular}




\section{Solution Techniques}

\subsection{Computational Aerodynamics}

CFD simulations were completed on all 3 geometries to characterize the aerodynamic behavior of the designs prior to wind tunnel or flight tests. This section briefly describes the methodology used for the simulations.

\subsubsection{Solver}

The double-precision solver of a commercially available code, $\mathrm{CFD}^{++} \mathrm{v} 12.1 .1,{ }^{9}$ is used for the CFD simulations. The basic numerical framework in the code contains unified-grid, unifiedphysics, and unified-computing features. A brief synopsis of this framework and methodology is given below. Additional information is available in Pulliam and Steger ${ }^{10}$ and in Peroomian et al. $^{11}$

The 3-dimensional (3-D) Reynolds-averaged Navier-Stokes (RANS) ${ }^{12}$ equations are solved using the following finite volume method:

$$
\frac{\partial}{\partial t} \int_{V} \boldsymbol{W} d V+\oint[\boldsymbol{F}-\boldsymbol{G}] \cdot d A=\int_{V} \boldsymbol{H} d V,
$$

where $\mathbf{W}$ is the vector of conservative variables; $\mathbf{F}$ and $\mathbf{G}$ are the inviscid and viscous flux vectors, respectively; $\mathbf{H}$ is the vector of source terms; $V$ is the cell volume; and $A$ is the surface area of the cell face.

The numerical framework of CFD++ is based on the following general elements: 1) unsteady compressible and incompressible Navier-Stokes equations with turbulence modeling (unifiedphysics); 2) unification of Cartesian, structured-curvilinear, and unstructured grids, including hybrids (unified-grid); 3) unification of treatment of various cell shapes, including hexahedral, tetrahedral, and triangular prism (i.e., 3-D) cells; quadrilateral and triangular (i.e., 2-dimensional [2-D]) cells and linear (i.e., 1-dimensional [1-D]) elements (unified-grid); 4) treatment of multiblock patched aligned (nodally connected), patched-nonaligned, and overset grids (unifiedgrid); 5) total variation diminishing discretization based on a new multidimensional interpolation framework; 6) Riemann solvers to provide proper signal propagation physics, including versions for preconditioned forms of the governing equations; 7) consistent and accurate discretization of viscous terms using the same multidimensional polynomial framework; 8) point-wise turbulence models that do not require knowledge of distance to walls; 9) versatile boundary condition implementation that includes a rich variety of integrated boundary condition types for the various sets of equations; and 10) implementation on massively parallel computers based on the distributed-memory message-passing model using native message-passing libraries or Message Passing Interface (MPI), Parallel Virtual Machine (PVM), etc., (unified-computing). 
The code has brought together several ideas on convergence acceleration to yield a fast steadystate solution methodology for all flow regimes. The approach can be labeled a "preconditionedimplicit-relaxation" scheme. It combines 3 basic ideas: implicit local time-stepping, relaxation, and preconditioning. Preconditioning the equations ideally equalizes the eigenvalues of the inviscid flux Jacobians and removes the stiffness arising from large discrepancies between the flow and sound velocities at low speeds. The use of an implicit scheme circumvents the stringent stability limits suffered by their explicit counterparts, and successive relaxation allows update of cells as information becomes available and, thus, aids convergence. The suggested ramping of the Courant-Friedrich-Lewy (CFL) number within $\mathrm{CFD}^{++}$also speeds convergence.

Only the subsonic regime $(0.3<M<0.8)$ is considered in this report, where the suggested CFL ramping is from 1.0 to 100.0 over 100 iterations. However, depending on the angle of attack being investigated, the ramping of the CFL number had to be limited to a value of 25 or 50 . Second-order discretization was used for the flow variables and the turbulent viscosity equation. Turbulence closure is based on topology-parameter-free formulations. Only 1 of the available turbulence models within $\mathrm{CFD}^{++}$was used in the current study, based on the results of the turbulence study in Silton and Fresconi ${ }^{4}$ : the Goldberg 3-equation k- $\varepsilon$-R turbulence model. ${ }^{13}$ This 3-equation model solves the transport equations for undamped eddy viscosity, $\mathrm{R}$, in addition to turbulent kinetic energy $(k)$ and its dissipation rate $(\varepsilon)$. This solution methodology accounts for non-equilibrium conditions and avoids freestream turbulence decay under shear-free flow conditions.

A tool exists within $\mathrm{CFD}^{++}$to recommend values for the initialization of the turbulence transport based on user specified free-stream turbulence intensity (or the turbulent-to-molecular viscosity ratio) and the turbulence length scale, which the model requires. For the current problem, the turbulence intensity was set to $2 \%$ and the turbulent-to-molecular viscosity ratio was set to 50 since the length scale was not known.

\subsubsection{Numerical Grids}

All grids used in the computational aerodynamics simulations were created using MIME v4.1. ${ }^{14}$ MIME is an unstructured mesh generator that allows triangular or quadrilateral dominant cells for the surface mesh. Once an adequate surface mesh is generated, prism layers can be specified and are created when the volume mesh is generated. MIME saves a parameter file with the surface names and desired cell sizes that can be utilized for creating new meshes with similar geometries (as in the present study). The user only needs to import the new geometry, open the old parameter file, and assign the geometry as appropriate. The grid employed here is based on the parameter file used to create the final mesh in Silton and Fresconi. ${ }^{4}$ As such, no further mesh refinement study was conducted.

Figure 2 shows the limits of the outer boundary of the mesh. It extends approximately 35 body lengths from the projectile in all directions. Cylindrical density boxes were placed in the wake of the projectile and the fins to ensure proper resolution in this area. An additional density box was 
placed between the canards and the fins to ensure that the canard tip vortices would not dissipate too much before reaching the fins. The surface mesh and boundary layer growth was restricted to a ratio of 1.2 or less. The growth ratio was relaxed to 2.0 when the remainder of the volume mesh was created. The prism layer spacing was chosen such that wall function spacing was present on the body, while a solve-to-wall mesh was used on the fins and canards. To achieve this end, a first cell spacing for the prism layer $(d y)$ was specified at $3.5 \times 10^{-3} \mathrm{~mm}$ on the fins and canards, $0.25 \mathrm{~mm}$ on the body, and $0.2 \mathrm{~mm}$ on the nose. MIME allowed for a smooth transition between the spacings.

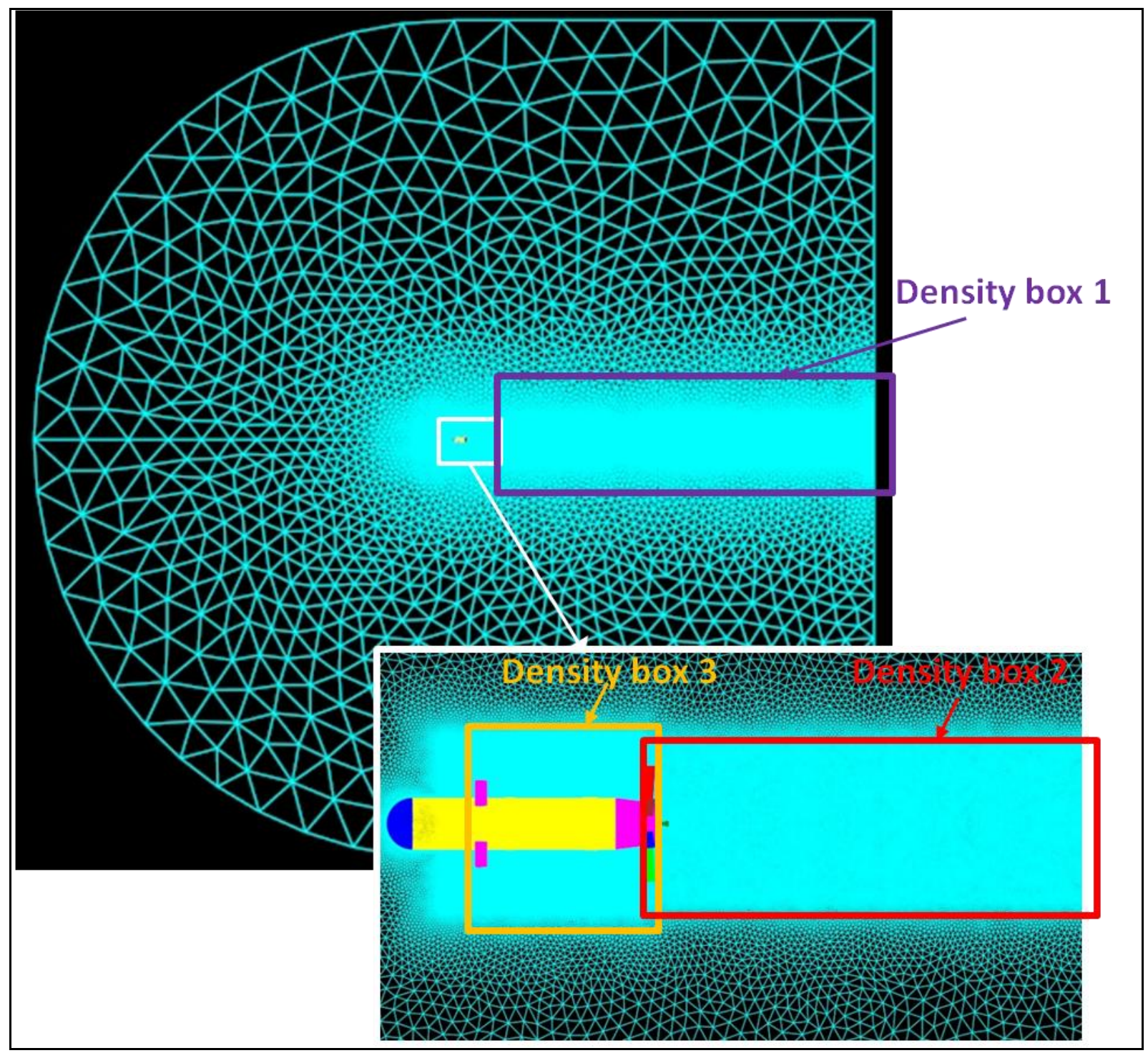

Fig. 2 Extent of outer mesh boundary with close-up of projectile showing locations of density boxes

\subsubsection{Flow Field and Boundary Conditions}

All computations were completed using a free-stream temperature and pressure of $288.15 \mathrm{~K}$ and $101325 \mathrm{~Pa}$, respectively. During the course of the study, up to 3 Mach numbers were 
investigated: Mach $0.50,0.65$, and 0.80 . Up to 14 angles of attack between $-14^{\circ}$ and $14^{\circ}$, inclusive, were also considered. The domain was initialized using free-stream conditions everywhere. For some cases, the area immediately surrounding the projectile was initialized with zero velocity to aid convergence.

The entire far-field boundary was set as "characteristic based" inflow/outflow. This boundary condition takes the specified free-stream conditions and solves a Riemann problem at the boundary using the supplied data as a virtual state outside the domain. The walls of the projectile were specified as adiabatic, no slip, viscous walls. The wall function integration option was chosen for the projectile surface unless the first prism layer was such to ensure proper integration to the wall, as was the case on the fins and canards.

\subsection{Parameter Sensitivity Technique}

The goal of the parameter sensitivity routine was to assess the maneuver characteristics of various candidates. Airframe input data (aerodynamics and mass properties) were perturbed to arrive at a hypothetically optimal configuration. Lift-to-drag ratio at the trim angle-of-attack was the key metric for optimization. Canard size, canard location, canard deflection, fin size, fin location, and center-of-gravity location were parameterized from the nominal values to derive theoretical configurations.

The approach for the parameter sensitivity routine was to begin by assembling the baseline mass properties and aerodynamics. The body, fin, and canard aerodynamics were considered separately to facilitate the optimization analysis and to allow the independent parameters of interest to be varied. These independent variables included canard and fin size (area), canard deflection angle, and canard hinge location. Once the baseline aerodynamic coefficients were specified, the canard and fin size could be altered through scaling of the axial and normal force coefficients. Additionally, the canard deflection angles, $\delta$, were accounted for by adding $\delta$ to $\alpha$ and determining the canard aerodynamics at the total canard angle. Finally, if canard location or center of gravity location was varied, the variation was accounted for by properly adjusting the pitching moment coefficient.

The sensitivity algorithm uses a given set of component aerodynamic coefficient derivatives as input for the airframe. Additionally, a range of values for each parameter is specified to determine if a better configuration can be designed within the limitations of the given aerodynamic coefficient database. The only constraint on the optimization is that a given configuration is stable (marginal stability is allowed). For each set of parameters, the pitching moment of the individual components are determined and then summed to obtain the total pitching moment for the parameter set as shown in Eq. 2. 


$$
\begin{aligned}
\sum C_{m}(M, \alpha) & =f_{F} C_{m}^{F}(M, \alpha)+C_{m}^{B}(M, \alpha)+f_{C}\left[C_{m}^{C}(M, \delta+\alpha)\right. \\
& \left.+\left(x_{c}-x_{c_{r e f}}\right) C_{N}^{C}(M, \delta+\alpha)\right]
\end{aligned}
$$

In this equation, $f_{C}$ and $f_{F}$ are the canard and fin scaling factor, respectively; $x_{c}$ is the canard hinge location forward of the $X_{c g} ; x_{c_{r e f}}$ is the canard hinge location at which the canard aerodynamic coefficients were determined; $q$ is the dynamic pressure; $S$ is the aerodynamic reference area; $D$ is the reference diameter; $C_{m}$ is the pitching moment; and the superscripts $F$, $B$, and $C$ represent the fin, body, and canard, respectively. The trim angle-of-attack $\left(\alpha_{\text {trim }}\right)$ was found by finding the angle for which Eq. 2 equals zero (i.e., $C_{m}$ changes sign). If no $\alpha_{\text {trim }}$ exists, the parameter set is considered to give an unstable design and therefore is not considered further.

The total axial and normal force, $C_{X}$ and $C_{N}$, respectively, at this $\alpha_{\text {trim }}$ was determined as shown in Eqs. 3 to 4 below.

$$
\begin{aligned}
& C_{N}\left(M, \alpha_{\text {trim }}\right)=f_{F} C_{N}^{F}\left(M, \alpha_{\text {trim }}\right)+C_{N}^{B}\left(M, \alpha_{\text {trim }}\right)+f_{C} C_{N}^{C}\left(M, \delta+\alpha_{\text {trim }}\right) \\
& C_{X}\left(M, \alpha_{\text {trim }}\right)=f_{F} C_{X}^{F}\left(M, \alpha_{\text {trim }}\right)+C_{N}^{B}\left(M, \alpha_{\text {trim }}\right)+f_{C} C_{X}^{C}\left(M, \delta+\alpha_{\text {trim }}\right)
\end{aligned}
$$

Finally, the lift-to-drag ratio $(L / D)$ was calculated from Eq. 5.

$$
L / D=\frac{C_{N}\left(M, \alpha_{\text {trim }}\right) \cos \left(\alpha_{\text {trim }}\right)-C_{X}\left(M, \alpha_{\text {trim }}\right) \sin \left(\alpha_{\text {trim }}\right)}{C_{N}\left(M, \alpha_{\text {trim }}\right) \sin \left(\alpha_{\text {trim }}\right)+C_{X}\left(M, \alpha_{\text {trim }}\right) \cos \left(\alpha_{\text {trim }}\right)}
$$

The optimal hypothetical design is determined by finding the combination of parameters that produce the maximum $L / D$ at its $\alpha_{\text {trim }}$. This sensitivity analysis is relatively general and can be applied to various aerodynamic surfaces with different aerodynamics models.

\subsection{Flight Mechanics and Control}

The controlled flight behavior of the different airframe configurations was investigated. Models of the flight, actuation, and measurements were used in flight control algorithms and implemented in simulation to assess performance. A linear state space representation $(\overrightarrow{\dot{x}}=\vec{A} \vec{x}+$ $\vec{B} \vec{u}+\vec{F}$ ) of the projectile flight with a first order model for the actuator is presented. The state vector is composed of the roll angle $(\phi)$; angular rates $(p, q, r)$; lateral accelerations $(\dot{v}, \dot{w})$; and deflections in roll $\left(\delta_{p}\right)$, pitch $\left(\delta_{q}\right)$, and yaw $\left(\delta_{r}\right)$ as shown in Eq. 6.

$$
\vec{x}=\left[\begin{array}{lllllllll}
\phi & p & q & r & \dot{v} & \dot{w} & \delta_{p} & \delta_{q} & \delta_{r}
\end{array}\right]^{T}
$$


The controls are the commanded deflections in roll $\left(\delta_{C, p}\right)$, pitch $\left(\delta_{C, q}\right)$, and yaw $\left(\delta_{C, r}\right)$ as shown in Eq. 7.

$$
\vec{u}=\left[\begin{array}{lll}
\delta_{C, p} & \delta_{C, q} & \delta_{C, r}
\end{array}\right]^{T}
$$

The system dynamics matrix, with mass properties, aerodynamic characteristics, and actuator parameters, is provided in Eq. 8 below

$$
\vec{A}=\left[\begin{array}{ccccccccc}
0 & 1 & 0 & 0 & 0 & 0 & 0 & 0 & 0 \\
0 & \frac{Q S D}{I_{x x}} \frac{D}{2 V} C_{l_{p}} & 0 & 0 & 0 & 0 & \frac{Q S D}{I_{z z}} C_{l_{\delta_{p}}} & 0 & 0 \\
0 & 0 & \frac{Q S D}{I_{z z}} \frac{D}{2 V} C_{m_{q}} & 0 & 0 & \frac{m D}{I_{z z}} \frac{C_{m_{\alpha}}}{C_{Z_{\alpha}}} & 0 & \frac{Q S D}{I_{z z}} C_{m_{\delta_{q}}} & 0 \\
0 & 0 & 0 & -\frac{Q S D}{I_{y y}} \frac{D}{2 V} C_{m_{r}} & \frac{m D}{I_{y y}} \frac{C_{n_{\beta}}}{C_{Y_{\beta}}} & 0 & 0 & 0 & -\frac{Q S D}{I_{y y}} C_{n_{\delta_{r}}} \\
0 & 0 & 0 & \frac{Q S}{m} C_{Y_{\beta}} & -\frac{Q S}{m V} C_{Y_{\beta}} & 0 & 0 & 0 & 0 \\
0 & 0 & \frac{Q S}{m} C_{Z_{\alpha}} & 0 & 0 & -\frac{Q S}{m V} C_{Z_{\alpha}} & 0 & 0 & 0 \\
0 & 0 & 0 & 0 & 0 & 0 & -\frac{1}{\tau_{p}} & 0 & 0 \\
0 & 0 & 0 & 0 & 0 & 0 & 0 & -\frac{1}{\tau_{q}} & 0 \\
0 & 0 & 0 & 0 & 0 & 0 & 0 & 0 & -\frac{1}{\tau_{r}}
\end{array}\right]
$$

The following expression for controls matrix (Eq. 9) contains the actuator time constant.

$$
\vec{B}=\left[\begin{array}{ccc}
\overrightarrow{0}_{6 \times 1} & \overrightarrow{0}_{6 \times 1} & \overrightarrow{0}_{6 \times 1} \\
\frac{1}{\tau_{p}} & 0 & 0 \\
0 & \frac{1}{\tau_{q}} & 0 \\
0 & 0 & \frac{1}{\tau_{r}}
\end{array}\right]
$$

The aerodynamic roll torque (Eq. 10) is the only term in the $\vec{F}$ matrix.

$$
\vec{F}=\left[\begin{array}{c}
0 \\
\frac{Q S D}{I_{x x}} C_{l_{0}} \\
\overrightarrow{0}_{7 \times 1}
\end{array}\right]
$$


Feedback of roll angle, angular rate sensors, and accelerometers was utilized in this guidance, navigation, and control scheme, which yields the measurement matrix in Eq. 11.

$$
\vec{C}=\left[\begin{array}{ll}
\vec{I}_{6 \times 6} & \overrightarrow{0}_{6 \times 3} \\
\overrightarrow{0}_{3 \times 6} & \overrightarrow{0}_{3 \times 3}
\end{array}\right]
$$

This linear model was implemented in simulation and then used with optimal control theory for preliminary flight control design of the high maneuverability airframe.

The nonlinear flight mechanics of these airframes was also studied. The 6 degree-of-freedom model is provided. The translational and rotational kinematic equations are shown in Eqs. 12 and 13 , respectively. Shorthand is used for trigonometric quantities in the equations.

$$
\begin{gathered}
{\left[\begin{array}{c}
\dot{x} \\
\dot{y} \\
\dot{z}
\end{array}\right]=\left[\begin{array}{ccc}
c_{\theta} c_{\psi} & s_{\phi} s_{\theta} c_{\psi}-c_{\phi} s_{\psi} & c_{\phi} s_{\theta} c_{\psi}+s_{\phi} s_{\psi} \\
c_{\theta} s_{\psi} & s_{\phi} s_{\theta} s_{\psi}+c_{\phi} c_{\psi} & c_{\phi} s_{\theta} c_{\psi}+s_{\phi} s_{\psi} \\
-s_{\theta} & s_{\phi} c_{\theta} & c_{\phi} c_{\theta}
\end{array}\right]\left[\begin{array}{c}
u \\
v \\
w
\end{array}\right]} \\
{\left[\begin{array}{c}
\dot{\phi} \\
\dot{\theta} \\
\dot{\psi}
\end{array}\right]=\left[\begin{array}{ccc}
1 & s_{\phi} t_{\theta} & c_{\phi} t_{\theta} \\
0 & c_{\phi} & -s_{\phi} \\
0 & s_{\phi} / c_{\theta} & c_{\phi} / c_{\theta}
\end{array}\right]\left[\begin{array}{l}
p \\
q \\
r
\end{array}\right]}
\end{gathered}
$$

The dynamic equations are given in Eqs. 14 and 15. The forces $(X, Y, Z)$ and moments $(L, M, N)$ acting on the projectile include both the aerodynamic and gravitational components.

$$
\begin{gathered}
{\left[\begin{array}{c}
\dot{u} \\
\dot{v} \\
\dot{w}
\end{array}\right]=\frac{1}{m}\left[\begin{array}{l}
X \\
Y \\
Z
\end{array}\right]-\left[\begin{array}{ccc}
0 & -r & q \\
r & 0 & -p \\
-q & p & 0
\end{array}\right]\left[\begin{array}{c}
u \\
v \\
W
\end{array}\right]} \\
{\left[\begin{array}{c}
\dot{p} \\
\dot{q} \\
\dot{r}
\end{array}\right]=\vec{I}^{-1}\left[\begin{array}{c}
L \\
M \\
N
\end{array}\right]-\vec{I}^{-1}\left[\begin{array}{ccc}
0 & -r & q \\
r & 0 & -p \\
-q & p & 0
\end{array}\right] \vec{I}\left[\begin{array}{l}
p \\
q \\
r
\end{array}\right]}
\end{gathered}
$$

The nonlinear models were implemented in simulation and enabled comprehensive flight control design. The flight mechanics and control for the high maneuverability airframe are provided in greater depth in Fresconi et al. ${ }^{16}$

\section{Results and Discussion}

The study presented herein was completed in 2 phases. The first phase of the study was carried out for the initial body-fin-canard configurations that were designed based on the results of the component build-up in Silton and Fresconi ${ }^{4}$ (i.e., aerodynamic coefficients of the body, fins, and canard components were determined separately and then summed for the total aerodynamic 
coefficients). In this phase of the study, CFD simulations were completed to determine the effect of the fin-canard interactions on the aerodynamic coefficients and, therefore, on the stability of the configuration, as well as on munitions in general. The aerodynamic results of the first phase were used as the aerodynamic model for the follow-on parameter sensitivity study. The resulting parameter set, to include fin and canard form factors and canard hinge location, was used as the basis for an exterior redesign.

In the second phase, CFD simulations were completed for the redesigned configuration, the results of which were used to further refine the aerodynamic model. Model refinement included, but was not limited to, investigating the use of higher order ballistic fits for the axial force and roll torque coefficients for each of the components (i.e., body, fins, individual canards) to determine if symmetry about $\alpha=0^{\circ}$ was appropriate, as this was not done in the first phase because lower order polynomial fits (i.e., symmetry was not enforced) were used. The refined aerodynamic model was first used for an additional parameter sensitivity study to ensure that there wasn't a better parameter set available. Finally, this refined aerodynamic model was used in a flight mechanics and control analysis to verify that the redesigned configuration would indeed meet the requirements of the demonstration platform.

\subsection{Phase 1}

The main purpose of the first phase of the study was to account for the effects of the fin-canard interactions on the aerodynamic coefficients, which had been neglected in previous studies. ${ }^{4}$ Aerodynamic computations were carried out based on designs similar to those in Silton and Fresconi ${ }^{4}$. However, the fin- and canard-scaling factors found to be optimal in Silton and

Fresconi ${ }^{4}$ could not be implemented due to physical constraints on the placement and size of the canards (i.e., $x_{C}=16.6 \mathrm{~mm}$ [0.2 cal.] from $X_{c g}$ was not achievable). As such, a slight redesign was performed prior to beginning this phase.

After completing a trade study, the decision was made to proceed forward with 2 configurations, Configuration 1a and Configuration $1 \mathrm{~b}$. The center of gravity location $\left(X_{c g}\right)$ was placed at 200 $\mathrm{mm}(2.41 \mathrm{cal})$ from the nose to be consistent with the previous study. Both configurations would have 4 rectangular fins with a 22.0-mm chord and 188.4-mm tip-to-tip span, corresponding to approximately $75 \%$ of the fin area used in Silton and Fresconi. ${ }^{4}$ A double bevel geometry was used to produce roll torque. Both configurations also had 4 NACA0015 canards, which were aligned with the fins. The canard chord was maintained at $18.86 \mathrm{~mm}$, but the canard span and dither axis location were allowed to vary. Configuration 1a had a tip-to-tip canard span of 240.48 $\mathrm{mm}$ and a canard axis location $68.89 \mathrm{~mm}$ (0.83 cal.) forward of $X_{c g}$ (Fig. 3, top). Configuration $1 \mathrm{~b}$ had a canard axis location $91.30 \mathrm{~mm}$ (1.1 cal.) forward of $X_{c g}$ and a 192.72-mm tip-to-tip span (Fig. 3). The tip-to-tip canard span for the canard aerodynamic data used for the parameter sensitivity study in Silton and Fresconi ${ }^{4}$ was approximately $191 \mathrm{~mm}$, so that the canard area in that study was similar $\left(\sim 100 \%\right.$ canard area, $\left.f_{C}=1.0\right)$ to the canard area in Configuration $1 \mathrm{~b}$. 


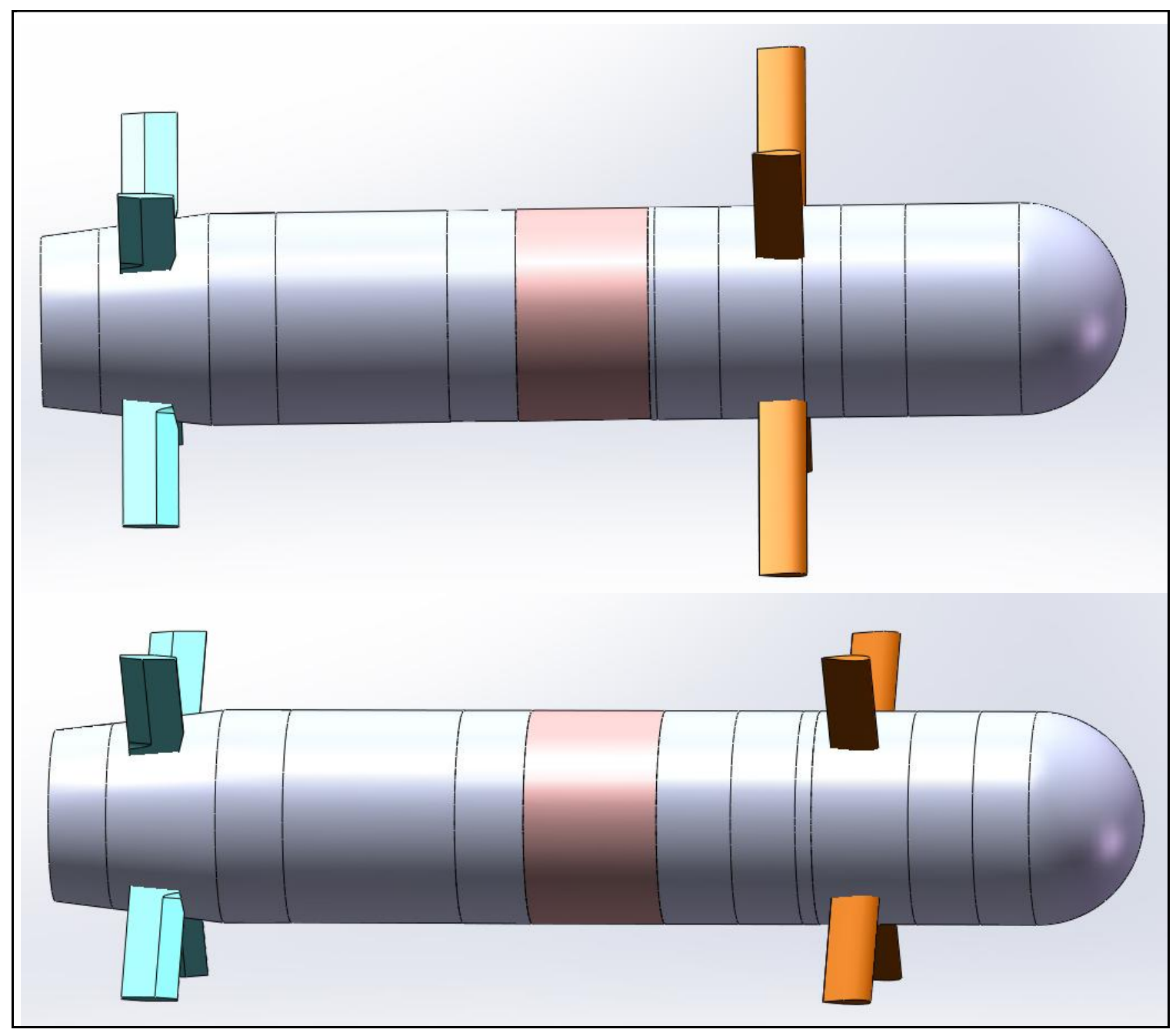

Fig. 3 Initial body-fin-canard geometries Configuration 1a (top) and Configuration 1b (bottom)

\subsubsection{Static Aerodynamic Characterization}

The static aerodynamic characterization of both initial configurations was completed for each of the 3 Mach numbers (Mach 0.5, 0.65, 0.8) at zero canard deflection angle. Seven angles-ofattack (positive angles only) were investigated for Configuration 1a (21 simulations) and 4 angles-of-attack (positive angles up to $10^{\circ}$ ) were investigated for Configuration $1 \mathrm{~b}(12$ simulations). For each simulation, a suitable number of iterations were completed (typically 2000 to 3000) to ensure convergence in both residual drop as well as in aerodynamic coefficients. The aerodynamic coefficients were obtained by averaging their values over the last 200 iterations to minimize any oscillations (mostly present at higher angles of attack). The simulations were completed on the IBM iDATAPlex system, Pershing, at the ARL DSRC. Using the meshing technique described in Section 3.1.2, a mesh containing approximately 33 million cells was created for both Configurations 1a and 1b. Each 33 million cell mesh was decomposed using pMETIS to run on 112 processors (approximately 295,000 cells per processor), which resulted in an average processing time of approximately $13 \mathrm{~s}$ per iteration. 
For each configuration, the axial force and roll torque coefficients $\left(C_{X}\right.$ and $C_{l}$, respectively) as well as normal force and pitching moment coefficients $\left(C_{N}\right.$ and $C_{m}$, respectively) were determined at each Mach number and each angle of attack. The forces and moments were examined for the complete configuration as well as for the contributions of the individual components (i.e., body, total fins, and each individual canard). The coefficient data for each of the components was transformed from the CFD coordinate system to the projectile body coordinate system. The coefficient data for the canards was further converted to the local blade coordinate system (the y-axis aligned with the blade, the z-axis perpendicular to the blade). The relative orientation of the canards is shown in Fig. 4; the roll orientation for Canard 4 is $45^{\circ}$ with the angle increasing in the clockwise direction (i.e., decreasing canard number).

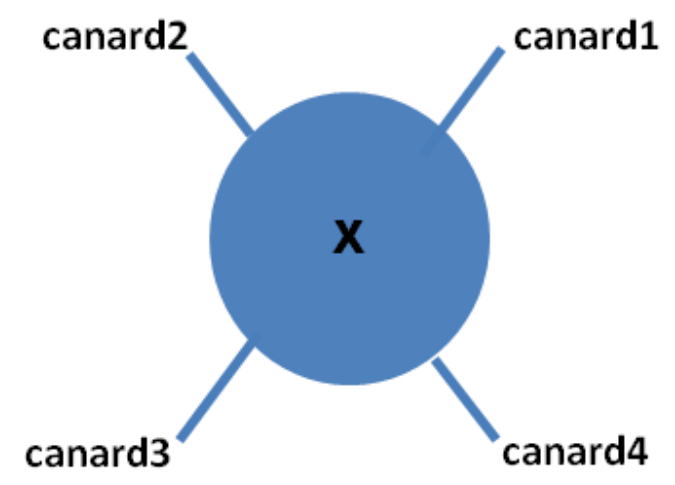

Fig. 4 Canard location looking from rear of projectile

A detailed explanation of these coordinate systems and coordinate transformations can be found in Appendix A. Polynomial and ballistic fits of the coefficients (Eqs. 16 to19) were then determined at each Mach number for each component.

$$
\begin{gathered}
C_{X}=C_{X_{0}}+C_{X_{\alpha 1}} \delta+C_{X_{\alpha 2}} \delta^{2}+C_{X_{\alpha 3}} \delta^{3}+C_{X_{\alpha 4}} \delta^{4} \\
C_{N}=C_{N_{\alpha}} \delta+C_{N_{\alpha 3}} \delta^{3} \\
C_{m}=C_{m_{\alpha}} \delta+C_{m_{\alpha 3}} \delta^{3} \\
C_{l}=C_{l_{0}}+C_{l_{\alpha 1}} \delta+C_{l_{\alpha 2}} \delta^{2}+C_{l_{\alpha 3}} \delta^{3}
\end{gathered}
$$

where

$$
\delta=\sin \alpha
$$

Figures 5 and 6 show the ability of the fits to capture the data trends at Mach 0.5 for the body and fin components and the individual canard components, respectively, for Configuration 1a. The fits were equally as good for the other Mach numbers as well as for Configuration 1b. A complete set of plots showing the coefficient fits can be found in Appendix B. It is important to 
remember that only positive body angles of attack were considered in the first phase.

Polynomial, rather than even ballistic fits, were implemented for $C_{X}$ and $C_{l}$ to ensure the best fit to the data. However, the use of polynomial fits does not guarantee symmetry about $\alpha=0^{\circ}$, which may be more realistic.

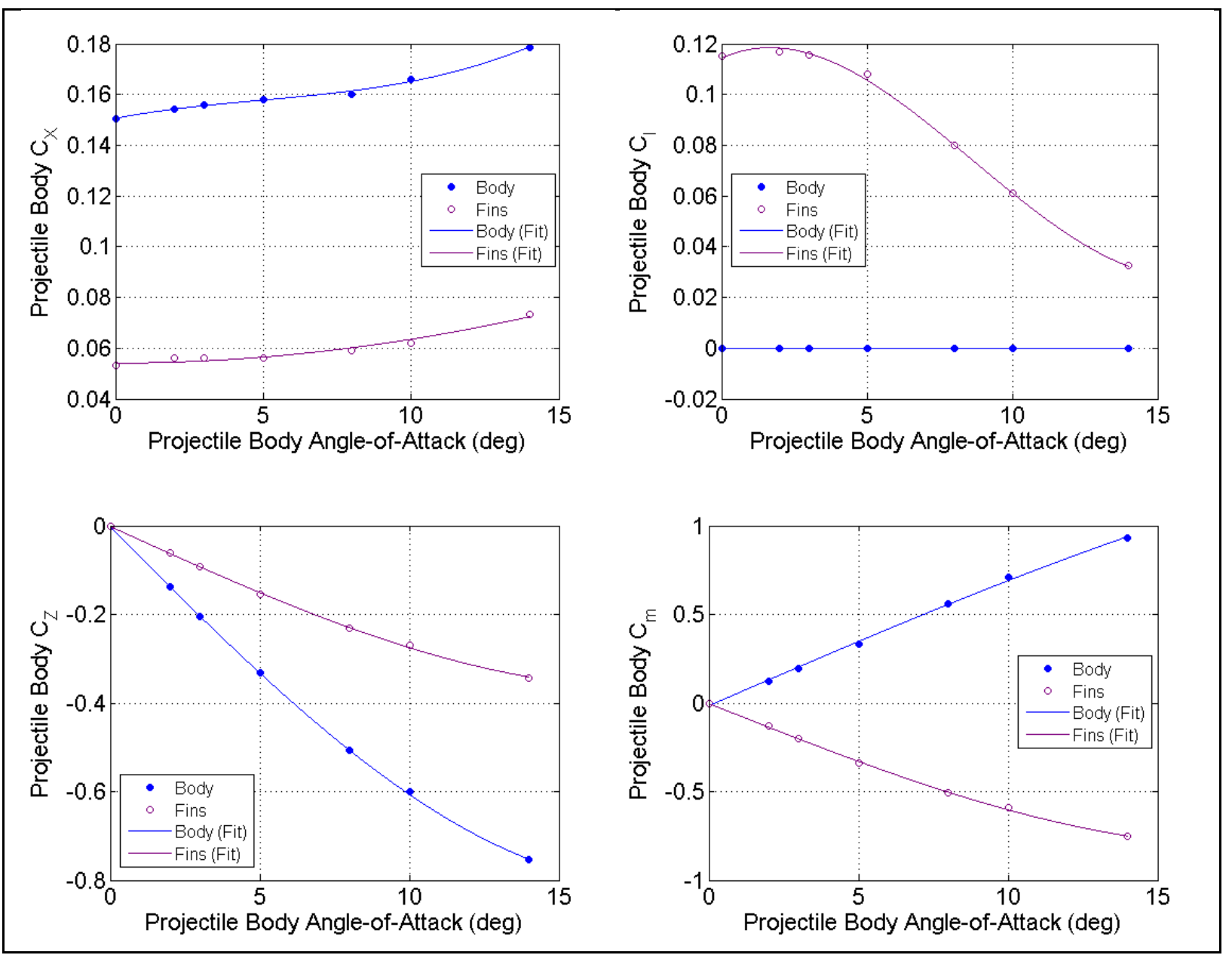

Fig. 5 Body and Fin data and fits in projectile body coordinate system for Configuration 1a, Mach 0.5

$C_{l}$ is fit for both the total fins and individual canards (body contribution is zero). However, the total canard contribution in the CFD simulations, as the canards are not deflected, is zero. The resulting fits for each component are converted to ballistic coordinates. Tables 2 and 3 list the values of coefficient derivatives in ballistic coordinates for both configurations for the body and fins components, respectively. For the fin component, only a second order ballistic fit was required to accurately fit the axial force coefficient, $C_{X}$; only $C_{X_{0}}$ and $C_{X_{\alpha 2}}$ are listed in Table 3 as the remainder of the coefficients have a value of zero.

Each of the canard aerodynamic fits was determined independently in the local canard coordinate system. This means Canards 2 and 3 have a negative local blade angle and Canards 1 and 4 have a positive local blade angle. As with the body and fin data, polynomial fits, rather than ballistic 
fits, were implemented for $C_{X}$ and $C_{l}$ to ensure the best fit to the data rather than forcing symmetry about $\alpha=0^{\circ}$. Tables 4 to 7 list the values of coefficient derivatives in ballistic coordinates (local canard coordinate system) for both configurations for each canard, respectively. There is no value listed for $C_{l_{0}}$ as it is always zero due to the canards not being deflected. These coefficient derivatives are used in Eqs. 16 to19 to determine the value of the coefficient attributable to each component at each Mach number for a given angle of attack.

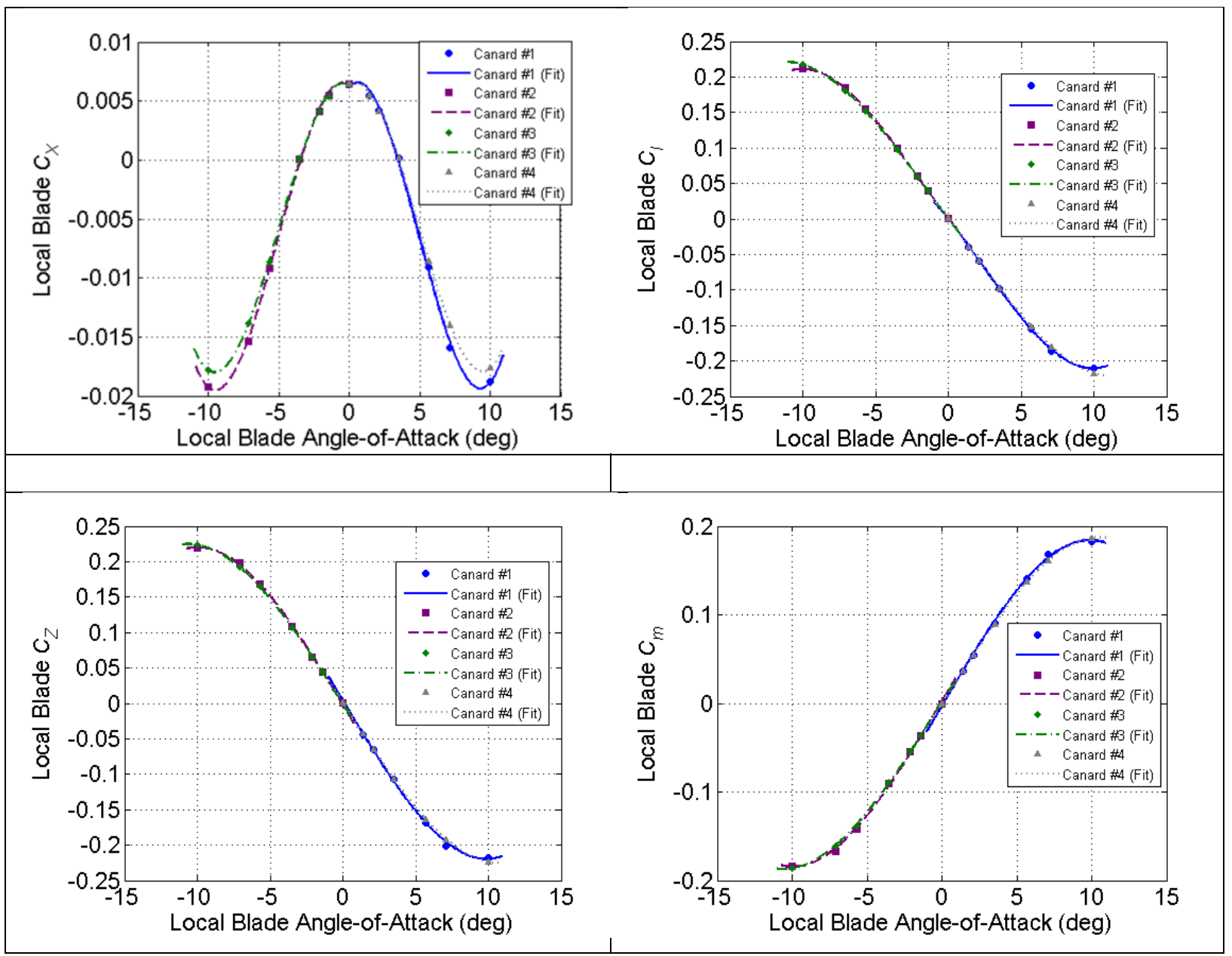

Fig. 6 CFD data and fits in for each canard in local canard coordinate system for Configuration 1a, Mach 0.5

The component coefficients are used as input for the flight dynamics model. During implementation, the canard contribution must be transformed from the local blade coordinate system back to the projectile body coordinate system. Figure 7 shows the results of this transformation for Configuration 1a at Mach 0.5. Because the canards are in an orientation that is $45^{\circ}$ off plane, the canard angle of attack in the local blade coordinate system is smaller than that in the projectile body coordinate system. Additionally, only a portion of the force along the canard z-axis is the Z-force direction of the projectile body. Therefore, the slopes of the coefficients are decreased during the transformation from the local blade coordinate system back to the body coordinate system. 
Table 2 Body component of aerodynamic coefficient fits for Configurations 1a and 1b

\begin{tabular}{|c|c|c|c|c|c|c|c|c|c|}
\hline Config. & Mach & $\boldsymbol{C}_{\boldsymbol{X}_{\mathbf{0}}}$ & $\boldsymbol{C}_{\boldsymbol{X}_{\boldsymbol{\alpha} \mathbf{1}}}$ & $\boldsymbol{C}_{\boldsymbol{X}_{\boldsymbol{\alpha} \mathbf{2}}}$ & $\boldsymbol{C}_{\boldsymbol{X}_{\boldsymbol{\alpha} \mathbf{3}}}$ & $\boldsymbol{C}_{\boldsymbol{N}_{\boldsymbol{\alpha}}}$ & $\boldsymbol{C}_{\boldsymbol{N}_{\boldsymbol{\alpha} \mathbf{3}}}$ & $\boldsymbol{C}_{\boldsymbol{m}_{\boldsymbol{\alpha}}}$ & $\boldsymbol{C}_{\boldsymbol{m}_{\boldsymbol{\alpha} 3}}$ \\
\hline \multirow{3}{*}{ 1a } & 0.50 & 0.1506 & 0.1282 & -0.7824 & 3.0321 & 3.8723 & -13.2237 & 4.1850 & -4.1115 \\
\cline { 2 - 10 } & 0.65 & 0.1482 & -0.0224 & 1.3919 & -3.1198 & 3.6772 & -13.6664 & 4.2459 & -5.8507 \\
\cline { 2 - 10 } & 0.80 & 0.2128 & 0.0124 & 2.4050 & -4.9798 & 2.7188 & 3.0697 & 3.9527 & -2.6088 \\
\hline \multirow{4}{*}{$1 \mathrm{~b}$} & 0.50 & 0.1529 & 0.0000 & 0.3501 & 0.0000 & 3.6358 & -10.9442 & 3.77247 & 12.3314 \\
\cline { 2 - 10 } & 0.65 & 0.1482 & 0.0000 & 0.7685 & 0.0000 & 3.8299 & -24.9079 & 4.1625 & -2.9340 \\
\cline { 2 - 10 } & 0.80 & 0.2140 & 0.0000 & 1.5736 & 0.0000 & 2.9240 & -7.5938 & 3.9940 & -3.7350 \\
\hline
\end{tabular}
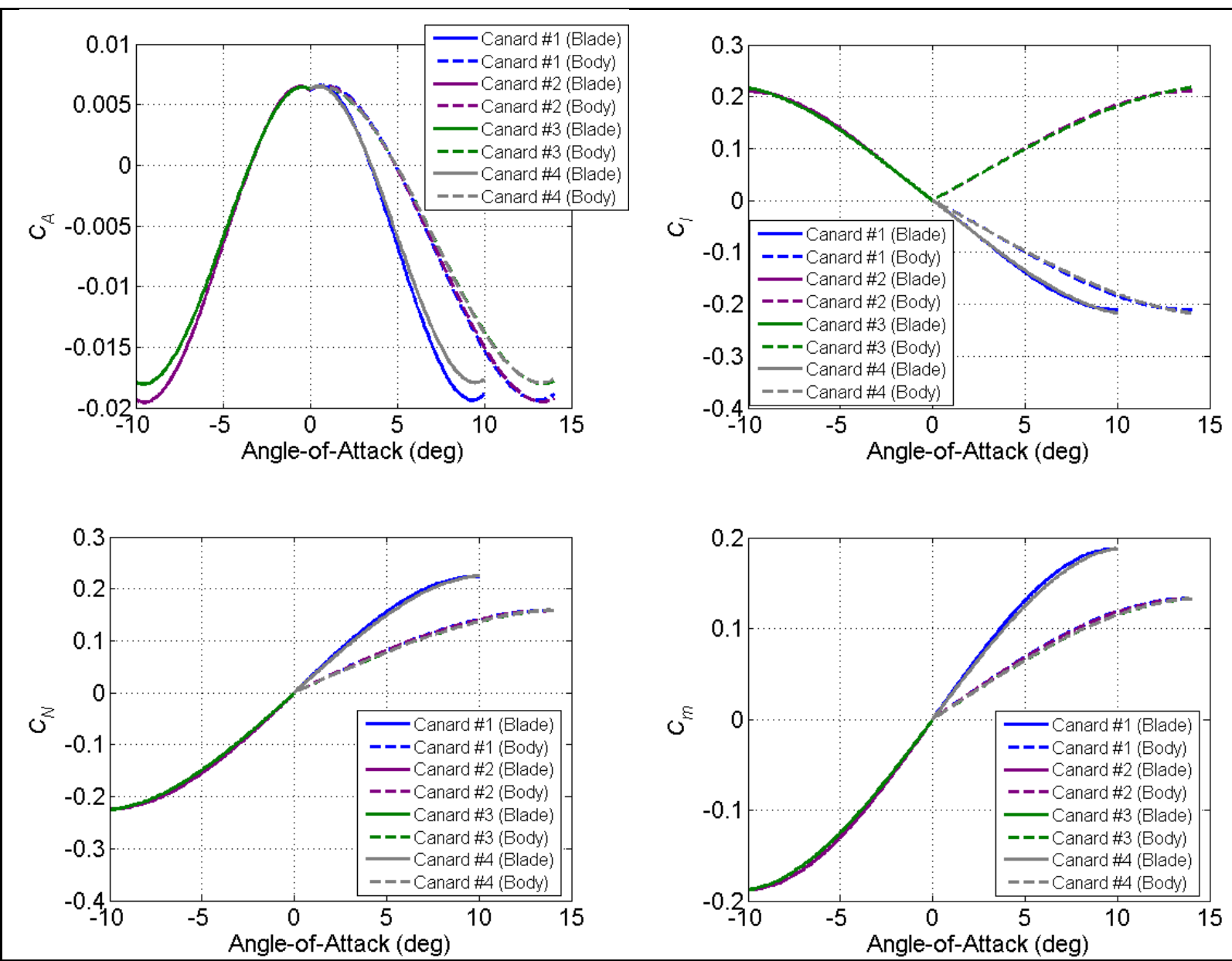

Fig. 7 Comparison of canard coefficients in local blade coordinate and projectile body coordinates for Configuration1a, Mach 0.5 
Table 3 Total fin component of aerodynamic coefficient fits for Configurations 1a and $1 \mathrm{~b}$

\begin{tabular}{|c|c|c|c|c|c|c|c|c|c|c|c|}
\hline Config. & Mach & $C_{X_{0}}$ & $C_{X_{\alpha 2}}$ & $C_{N_{\alpha}}$ & $C_{N_{\alpha 3}}$ & $C_{m_{\alpha}}$ & $C_{m_{\alpha 3}}$ & $C_{l_{0}}$ & $C_{l_{\alpha 1}}$ & $C_{l_{\alpha 2}}$ & $C_{l_{\alpha 3}}$ \\
\hline \multirow{3}{*}{$1 \mathrm{a}$} & 0.50 & 0.05413 & 0.31131 & 1.75757 & -6.0255 & -3.8427 & 12.8555 & 0.1143 & 0.2975 & -5.6023 & 12.2867 \\
\hline & 0.65 & 0.05536 & 0.39140 & 1.72327 & -5.3884 & -3.7730 & 11.4362 & 0.1196 & 0.2860 & -6.6319 & 16.1398 \\
\hline & 0.80 & 0.05662 & 0.55466 & 1.74705 & -5.6446 & -3.8301 & 11.9842 & 0.1200 & -0.1006 & -3.6367 & 10.9799 \\
\hline \multirow{3}{*}{$1 \mathrm{~b}$} & 0.50 & 0.05424 & 0.27124 & 1.9314 & -10.5717 & -4.2027 & 21.9989 & 0.1148 & 0.1578 & -2.9050 & 0.37678 \\
\hline & 0.65 & 0.05485 & 0.43284 & 1.85519 & -8.7736 & -4.0443 & 18.1562 & 0.1198 & 0.2025 & -5.1952 & 9.9199 \\
\hline & 0.80 & 0.05484 & 0.75421 & 1.82664 & -7.9024 & -4.0004 & 16.6889 & 0.1202 & 0.06957 & -6.9581 & 24.7248 \\
\hline
\end{tabular}

Table 4 Aerodynamic coefficient fits Canard 1 for Configurations 1a and $1 \mathrm{~b}$

\begin{tabular}{|c|c|c|c|c|c|c|c|c|c|c|c|c|c|}
\hline Config. & Mach & $C_{X_{0}}$ & $C_{X_{\alpha 1}}$ & $C_{X_{\alpha 2}}$ & $C_{X_{\alpha 3}}$ & $C_{X_{\alpha 4}}$ & $C_{N_{\alpha}}$ & $C_{N_{\alpha 3}}$ & $C_{m_{\alpha}}$ & $C_{m_{\alpha 3}}$ & $C_{l_{\alpha 1}}$ & $C_{l_{\alpha 2}}$ & $C_{l_{\alpha 3}}$ \\
\hline \multirow{3}{*}{$1 \mathrm{a}$} & 0.50 & 0.00614 & 0.0776 & -3.879 & 14.984 & 0.0 & 1.953 & -22.118 & 1.638 & -18.555 & -1.474 & -4.214 & 32.995 \\
\hline & 0.65 & 0.00616 & 0.0712 & -5.558 & 52.944 & -137.508 & 1.845 & -32.249 & 1.561 & -27.530 & -2.165 & 6.602 & 4.458 \\
\hline & 0.80 & 0.0121 & -0.00589 & 0.760 & -2.102 & -4.745 & 0.817 & -2.991 & 0.708 & -3.413 & -0.832 & 1.273 & -2.724 \\
\hline \multirow[t]{2}{*}{$1 b$} & 0.65 & 0.00410 & 0.0182 & -2.178 & 13.730 & 0.0 & 1.339 & -32.392 & 1.530 & -36.963 & -0.909 & -3.220 & 41.574 \\
\hline & 0.80 & 0.00718 & 0.00287 & 0.177 & 0.928 & 0.0 & 0.709 & -14.676 & 0.826 & -17.460 & -0.630 & 2.331 & -3.268 \\
\hline
\end{tabular}

Table 5 Aerodynamic coefficient fits Canard 2 for Configurations 1a and 1b

\begin{tabular}{|c|c|c|c|c|c|c|c|c|c|c|c|c|c|}
\hline Config. & Mach & $C_{X_{0}}$ & $C_{X_{\alpha 1}}$ & $C_{X_{\alpha 2}}$ & $C_{X_{\alpha 3}}$ & $C_{X_{\alpha 4}}$ & $C_{N_{\alpha}}$ & $C_{N_{\alpha 3}}$ & $C_{m_{\alpha}}$ & $\boldsymbol{C}_{\boldsymbol{m}_{\alpha 3}}$ & $C_{l_{\alpha 1}}$ & $C_{l_{\alpha 2}}$ & $C_{l_{\alpha 3}}$ \\
\hline \multirow{3}{*}{ 1a } & 0.50 & 0.00622 & 0.0650 & -3.622 & -13.832 & 0.0 & 1.934 & -21.547 & 1.621 & -18.058 & -1.503 & 3.616 & 30.426 \\
\hline & 0.65 & 0.00617 & -0.0701 & -5.549 & -53.139 & -138.790 & 1.837 & -32.469 & 1.555 & -27.689 & -2.177 & -6.852 & 3.775 \\
\hline & 0.80 & 0.0121 & 0.00526 & 0.736 & \begin{tabular}{|l|}
1.909 \\
\end{tabular} & -5.338 & 0.777 & -2.302 & 0.678 & -2.892 & -0.810 & -1.425 & -3.775 \\
\hline \multirow{3}{*}{$1 \mathrm{~b}$} & & 00416 & \begin{tabular}{l|l}
0.0 \\
\end{tabular} & -0.947 & 0.0 & 0.0 & 1.134 & -5.152 & 1.291 & -5.724 & -0.880 & 0.113 & 4.215 \\
\hline & 0.65 & 0.00410 & -0.0173 & -2.153 & -13.505 & 0.0 & 1.339 & -32.640 & 1.530 & -37.196 & -0.909 & 3.223 & 41.834 \\
\hline & 0.80 & 0.00718 & -0.00316 & 0.103 & -1.356 & 0.0 & 0.693 & -14.195 & 0.809 & -16.923 & -0.611 & -2.164 & --2.623 \\
\hline
\end{tabular}


Table 6 Aerodynamic coefficient fits Canard 3 for Configurations 1a and $1 \mathrm{~b}$

\begin{tabular}{|c|c|c|c|c|c|c|c|c|c|c|c|c|c|}
\hline Config. & Mach & $C_{X_{0}}$ & $C_{X_{\alpha 1}}$ & $C_{X_{\alpha 2}}$ & $C_{X_{\alpha 3}}$ & $C_{X_{\alpha 4}}$ & $C_{N_{\alpha}}$ & $C_{N_{\alpha 3}}$ & $C_{m_{\alpha}}$ & $C_{m_{\alpha 3}}$ & $C_{\alpha}$ & $C_{l_{\alpha 2}}$ & $C_{l_{\alpha 3}}$ \\
\hline \multirow{3}{*}{$1 \mathrm{a}$} & 0.50 & 0.00630 & -0.0468 & -3.217 & -12.367 & 0.0 & 1.837 & -18.004 & 1.540 & -15.278 & -1.578 & 1.318 & 18.452 \\
\hline & 0.65 & 0.00616 & -0.0725 & -5.716 & -55.545 & -148.299 & 1.773 & -27.253 & 1.498 & -23.442 & -2.127 & -6.714 & -1.650 \\
\hline & 0.80 & 0121 & -0.00467 & 1.043 & 7.837 & 16.518 & 0.814 & 1.451 & 0.702 & 0.154 & -0.6 & 2.104 & 5.684 \\
\hline \multirow{3}{*}{$1 b$} & & 10 & 0.0 & -0.882 & 0.0 & 0.0 & 1.128 & -6.360 & 1.283 & -7.208 & -0.8 & 0.0574 & 4.443 \\
\hline & 0.65 & 0.00410 & -0.0173 & -2.175 & -13.954 & 0.0 & 1.322 & -31.225 & 1.510 & -35.773 & -0.916 & 2.717 & 36.639 \\
\hline & 0.80 & 0.00718 & -0.00914 & 0.657 & 3.425 & 0.0 & 0.573 & -1.833 & 0.672 & -3.218 & -0.575 & -2.789 & -15.596 \\
\hline
\end{tabular}

Table 7 Aerodynamic coefficient fits Canard 4 for Configurations 1a and $1 \mathrm{~b}$

\begin{tabular}{|c|c|c|c|c|c|c|c|c|c|c|c|c|c|}
\hline Config. & Mach & $c_{X_{0}}$ & $C_{X_{\alpha 1}}$ & $C_{X_{\alpha 2}}$ & $C_{X_{\alpha 3}}$ & $C_{X_{\alpha 4}}$ & $C_{N_{\alpha}}$ & $C_{N_{\alpha 3}}$ & $C_{m_{\alpha}}$ & $C_{m_{\alpha 3}}$ & $C_{l_{\alpha 1}}$ & $C_{l_{\alpha 2}}$ & $C_{l_{\alpha 3}}$ \\
\hline \multirow{3}{*}{$1 \mathrm{a}$} & 0.50 & 0.00628 & 0.0514 & -3.307 & 12.763 & 0.0 & 1.841 & -18.032 & 1.544 & -15.320 & -1.574 & -1.418 & 18.812 \\
\hline & 0.65 & 0.00617 & 0.0708 & -5.640 & 54.752 & -145.926 & 1.772 & -26.793 & 1.497 & -23.067 & -2.128 & 6.760 & -2.299 \\
\hline & 0.80 & 0.0121 & 0.00283 & 1.134 & -8.612 & 18.497 & 0.841 & 1.245 & 0.723 & 0.0124 & -0.701 & -1.891 & 4.894 \\
\hline \multirow{3}{*}{$1 b$} & 0.50 & 0.00410 & 0.0 & -0.874 & 0.0 & 0.0 & 1.129 & -6.577 & 1.284 & -7.459 & -0.876 & -0.135 & 5.013 \\
\hline & 0.65 & 0.00410 & 0.183 & -2.204 & 14.192 & 0.0 & 1.325 & -31.317 & 1.514 & -35.906 & -0.914 & -2.797 & 37.132 \\
\hline & 0.80 & 0.00718 & 0.00968 & 0.653 & -3.381 & 0.0 & 0.597 & -2.485 & 0.698 & -3.927 & -0.581 & 2.521 & -13.786 \\
\hline
\end{tabular}


Once the component contributions have been determined, the total aerodynamic forces and moments can be determined by adding these contributions together (i.e., component build up). For this study, the component contributions include the interference effects between the components; they are not considered separately. As can be seen from Fig. 8 (Configuration 1a, Mach 0.65) and Fig. 9 (Configuration 1b, Mach 0.65), when the component fits are added together, there is agreement with the total coefficients obtained from CFD, which validates that the correct component fits are being used. (All of component fit build ups for Configurations 1a and $1 \mathrm{~b}$ can be found in Appendix B). Note, $C_{A}$ is used for axial force coefficient in these figures, rather than $C_{X}$, as the aerodynamic forces are being represented as the ballistic coefficients. However, it is quite apparent that neither of these configurations is statically stable (i.e., $C_{m}>0$ ) as was predicted during the trade study completed while designing the projectile. The trade study used the coefficients obtained from body-fin simulations and the previously obtained canard data presented in Silton and Fresconi. ${ }^{4}$ The question, of course, is why the trade study predicted different results than were determined by the CFD simulations.

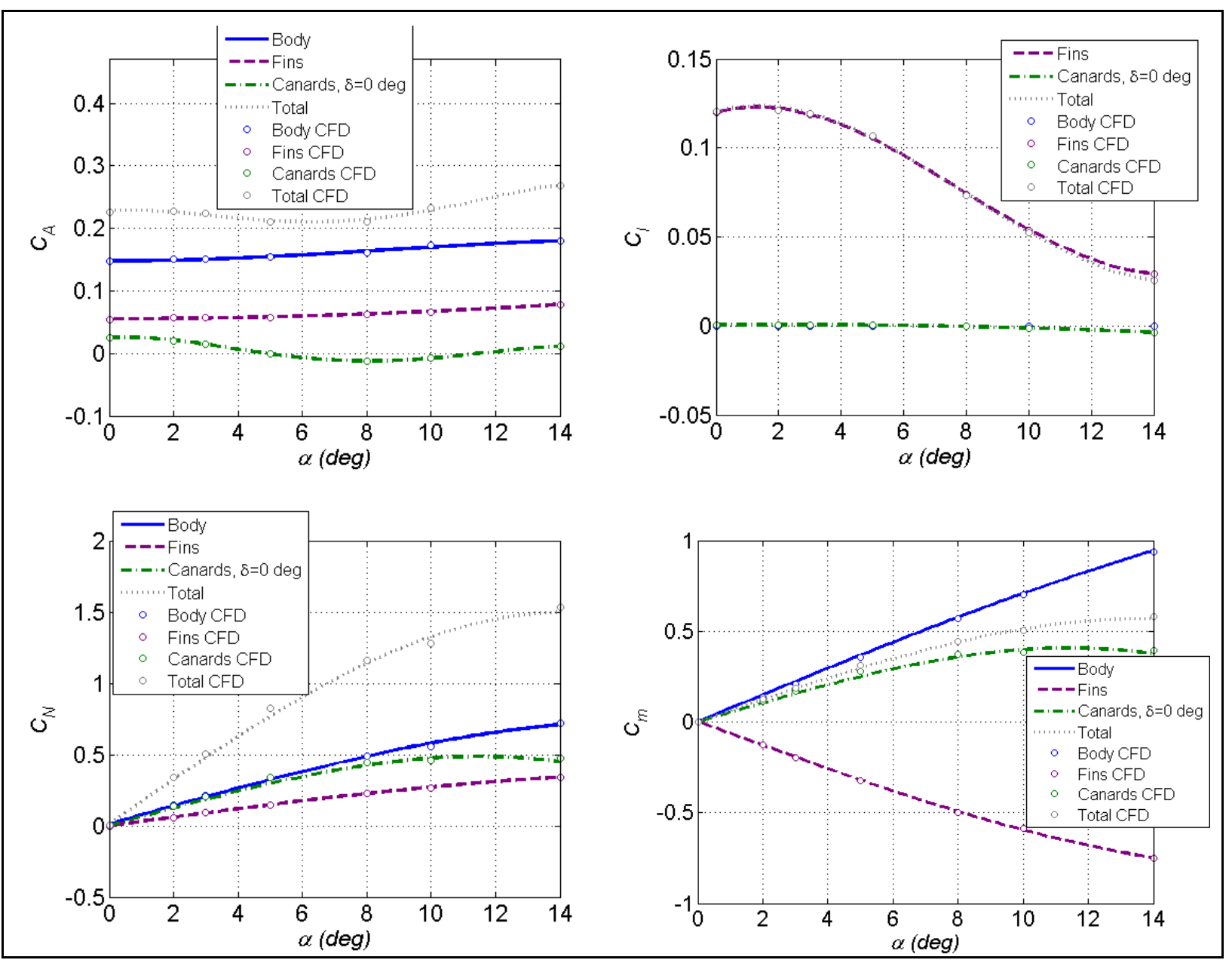

Fig. 8 Component fit build up comparison to total CFD coefficients for Configuration 1a, Mach 0.65 


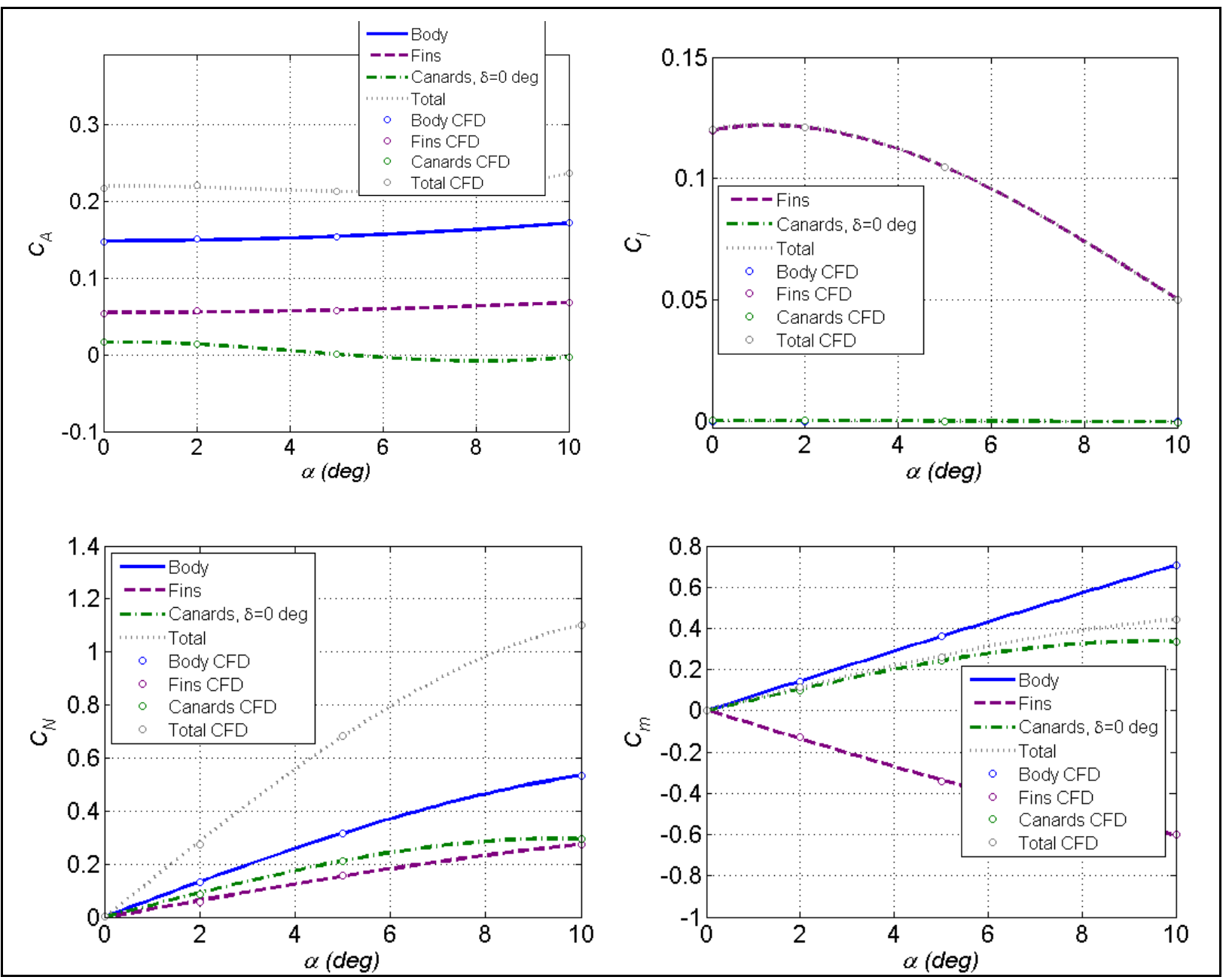

Fig. 9 Component fit build up comparison to total CFD coefficients for Configuration 1b, Mach 0.65

The lack of airframe stability can be answered by comparing the component aerodynamic contributions found during the trade study to those component aerodynamic contributions determined directly from the CFD (Fig. 9); specifically the body and fin components. The results of the trade study, which used the appropriately scaled body, fin, and canard aerodynamic coefficients (75\% fin effectiveness, $100 \%$ canard effectiveness) from Silton and Fresconi ${ }^{4}$ to correspond to Configuration 1b, are shown in Fig. 10. No canard-fin interaction effects are included in the trade study. The differences between the aerodynamic contributions of the body and fins are quite apparent. At $a=2^{\circ}$, there was less fin force (20\%) and moment (45\%) predicted by the CFD for the body-fin-canard model than the body-fin model (Fig. 11), which can likely be attributed to the interaction effects of the canard tip vortices with the fin blades. Additionally, there are increases in body normal force (20\%) and pitching moment (60\%) (see Fig. 12), which are also likely due to the presence of the canards. These results show just how important it is that interaction effects be accounted for when creating the aerodynamic model. It is obvious that the stability of the projectile may not be as expected if the canard-fin or the canard-body interaction effect is neglected. Finally, the canards themselves show a maximum of 
a $25 \%$ increase in normal force and pitching moments at angles of attack below $7^{\circ}$ (Fig. 13), which caused the projectile to be less stable than expected.

This large of a variation in the canard contribution over the experimental values used in Silton and Fresconi ${ }^{4}$ was a bit surprising; the same interaction effects causing the increase in body normal force and pitching moment may be causing the increased canard effectiveness. It is also possible that the different body shape (ogival rather than cylindrical) on which the canards were mounted in the experiment is causing the differences in canard aerodynamic forces. The differences become more significant above $\alpha=7^{\circ}$, as the slope of the canard component from the CFD appears to be decreasing, which indicates that aerodynamic stall may be beginning to occur, while the experimental data used in the component build-up indicates that aerodynamic stall does not occur until nearly $\alpha=12^{\circ}$. Wind tunnel tests will be conducted in the future to investigate the effect of the body shape and the onset of aerodynamic stall. 

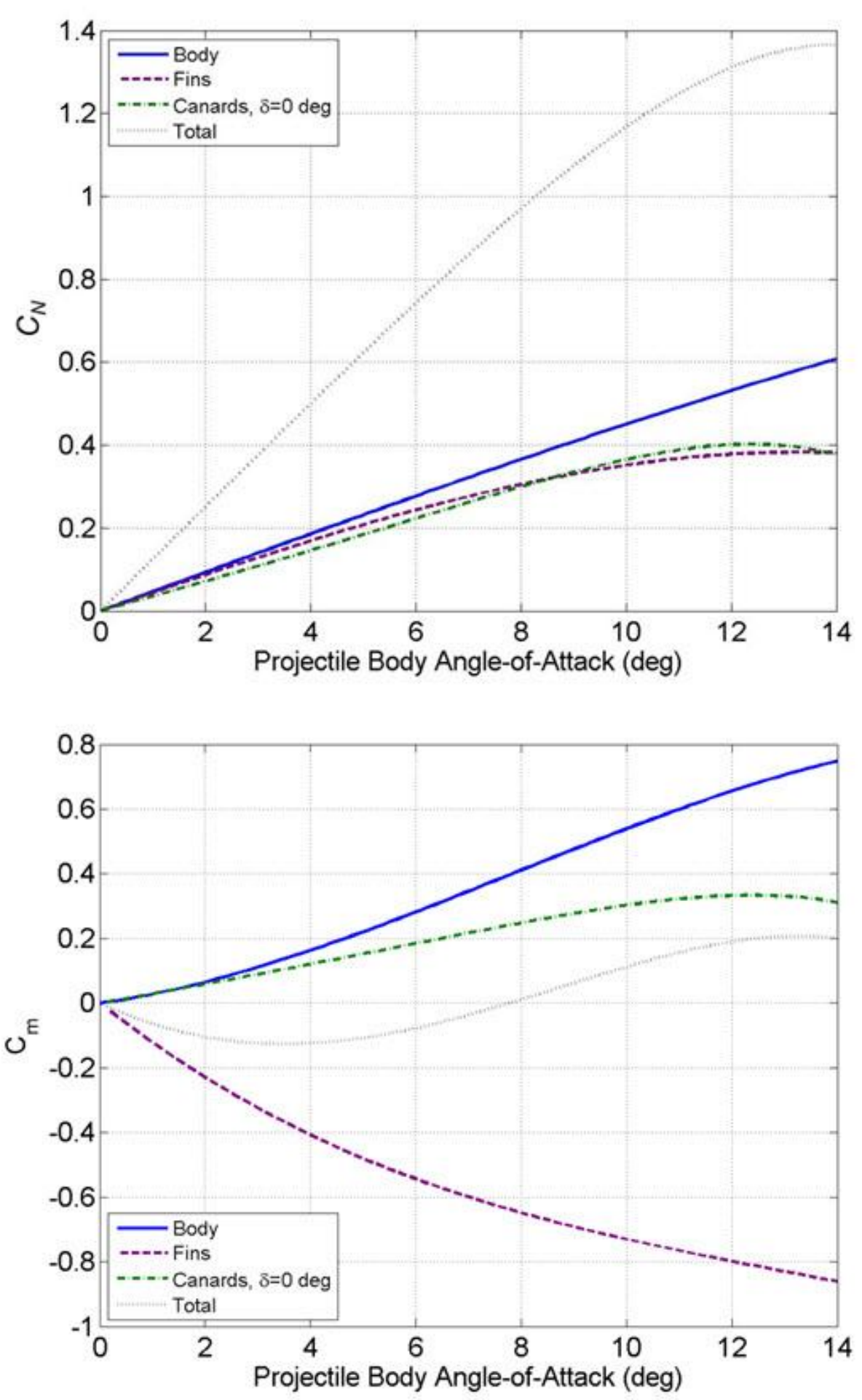

Fig. 10 Component build up of normal force and pitching moment using body-fin CFD and canard fit data from Silton and Fresconi with appropriate form factors to approximate Configuration 1b, Mach $0.65\left(f_{F}=0.75, f_{c}=1.0\right)$ 

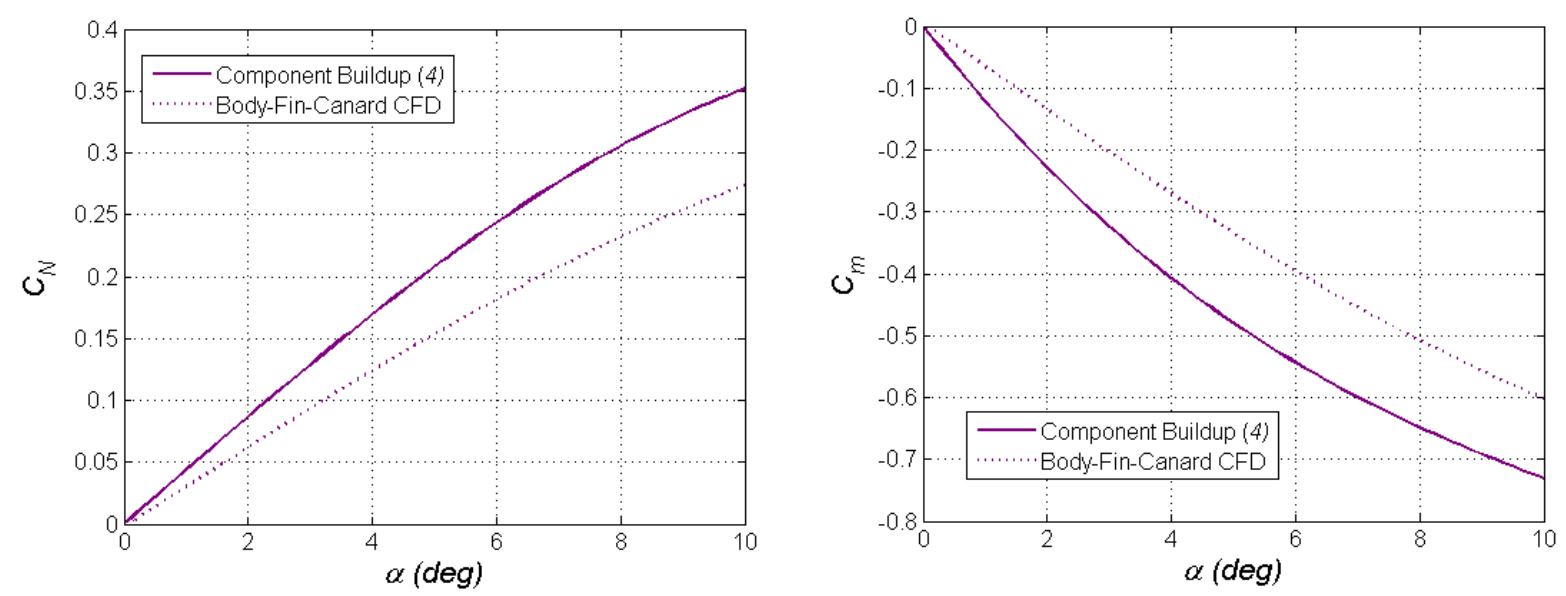

Fig. 11 Comparison of fin component as determined from component buildup with form factors and CFD simulation for Configuration 1b, Mach 0.65.
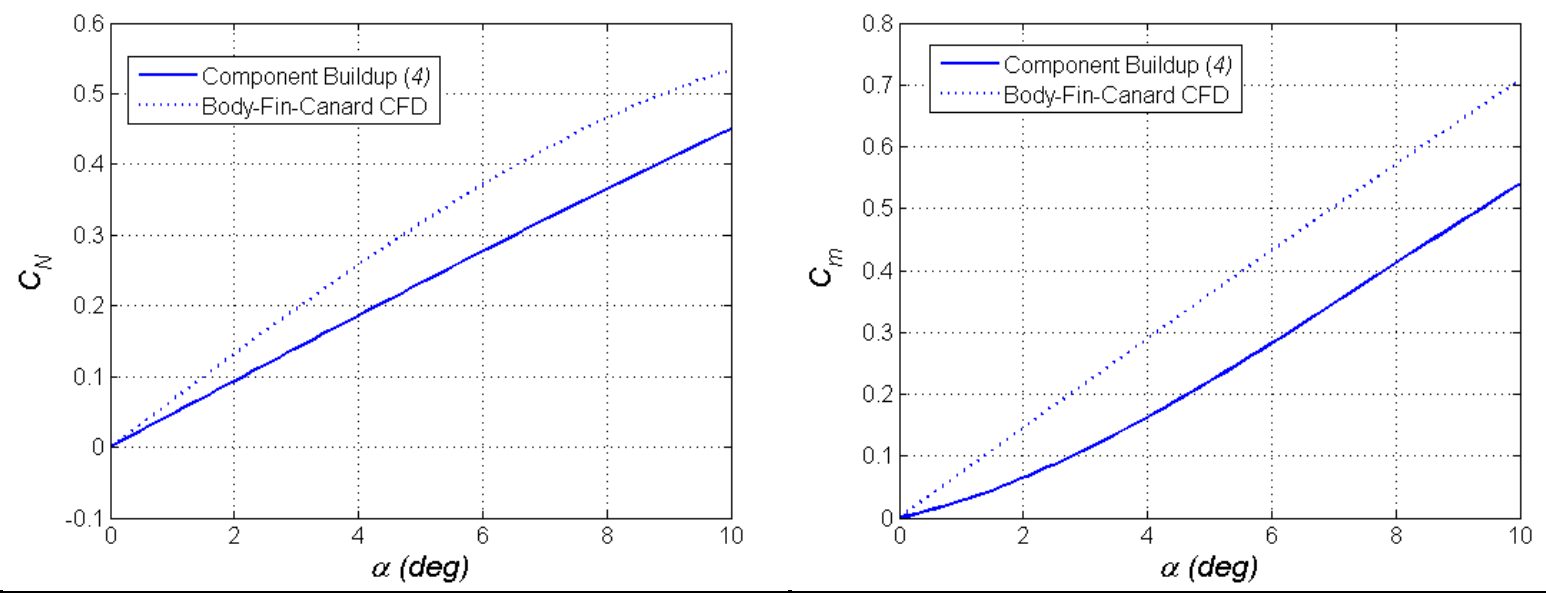

Fig. 12 Comparison of body component as determined from component buildup with form factors and CFD simulation for Configuration 1b, Mach 0.65 

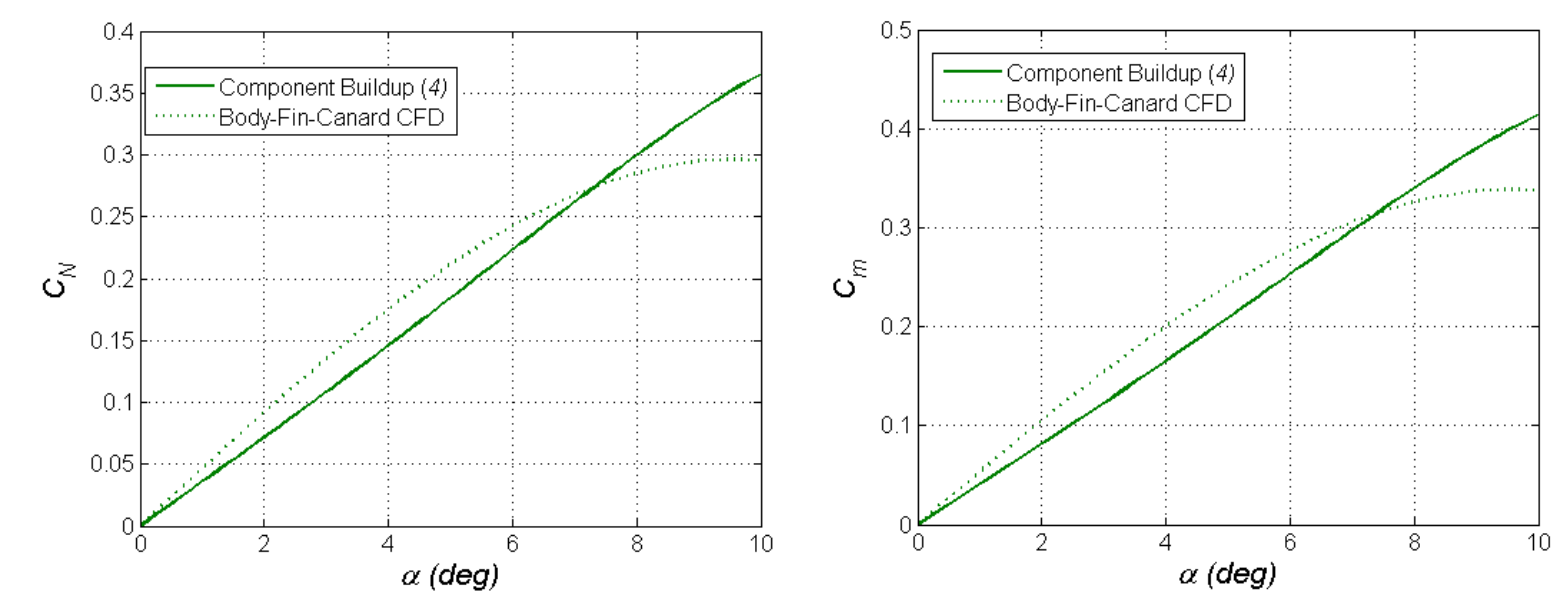

Fig. 13 Comparison of total canard component as determined from component buildup with form factors and CFD simulation for Configuration 1b, Mach 0.65

\subsubsection{Redesign for Static Stability and Parameter Sensitivity Study}

The aerodynamic data for both Configurations $1 \mathrm{a}$ and $1 \mathrm{~b}$ were used as input to the numerical implementation of Eqs. 2 to5 to redesign the airframe to ensure static stability while maximizing lift-to-drag ratio. The goal was to apply the results of a high fidelity aerodynamic characterization, which included the flow interaction effects, to obtain attractive airframe specifications. A sensitivity study was performed to determine the responsiveness of the designs to variations in fin size and canard size and location. Canard deflection angle was also varied to determine the trim angle that would maximize the lift-to-drag ratio. Because the fin aerodynamics included interaction effects due to the canards, scaling the fin area (through $f_{F}$ ) scaled the interaction effects as well.

Aerodynamic data were only available for the non-deflected canard case. However, data for the deflected canard case were necessary to use the equations. Because the canard coefficient fits were determined in the local blade coordinate system, the deflection angle is added to the local angle of attack; this total angle of attack is then used to determine the aerodynamic coefficient of the canard. Figure 14 shows the results of this methodology to determine the total coefficients assuming a deflection angle of $4^{\circ}$ for a pitch maneuver (i.e., all deflected "up") using Configuration 1a. While this method is not exact, it is a reasonable assumption at this time. Future studies will investigate its validity. Additionally, canard stall was accounted for by holding the canard normal force and pitching moment constant for total angles of attack outside the range for which CFD was completed, which is the reason the values of $C_{N}$ and $C_{m}$ remain constant above a body angle of attack of approximately $8^{\circ}$. 


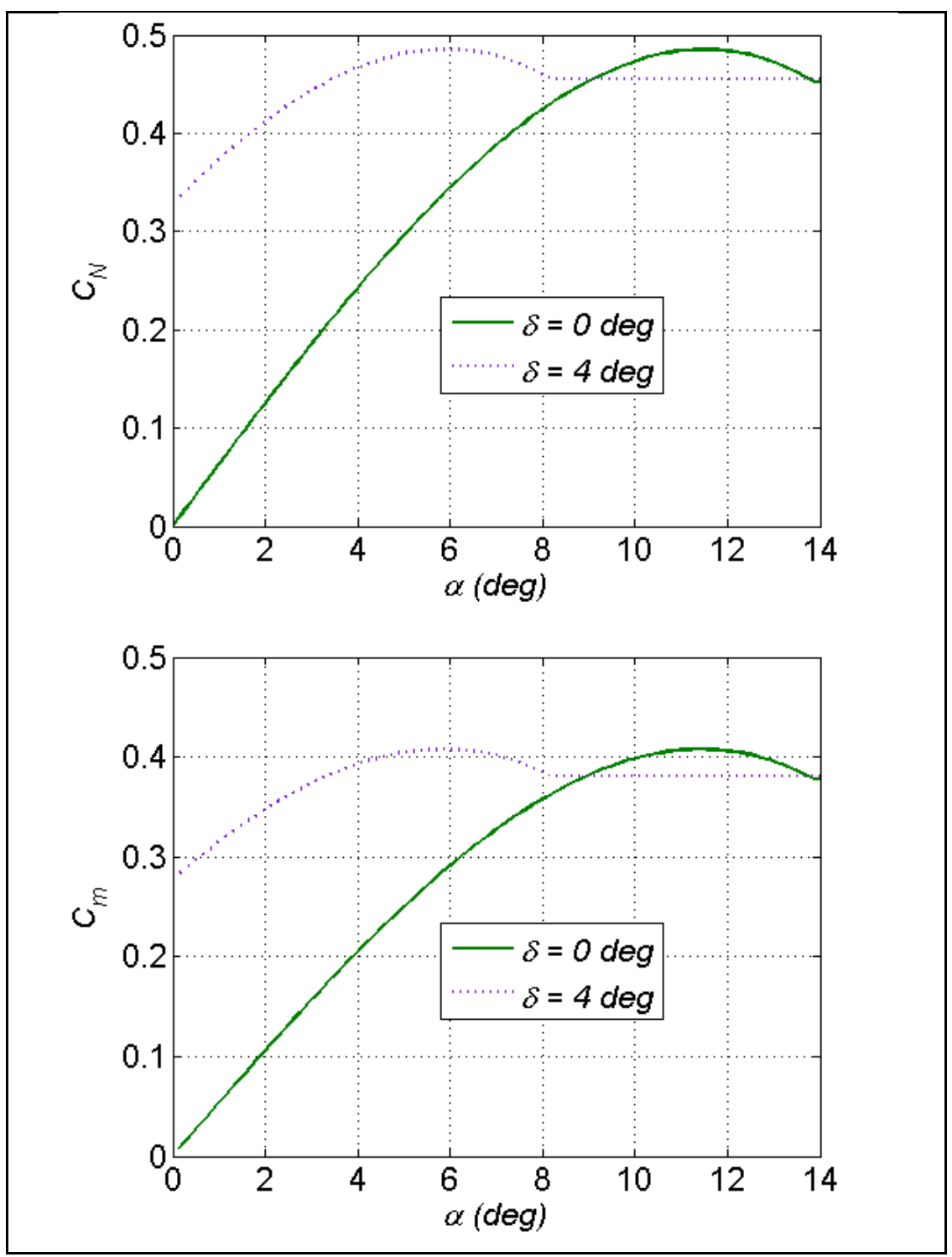

Fig. 14 Component fit build up of normal force and pitching moment with $4^{\circ}$ canard deflection for Configuration 1a, Mach 0.65

Configuration 1a is statically unstable, which necessitates larger fins and smaller canards. As such, the parameter sensitivity routine was executed using the aerodynamic coefficients obtained from CFD simulations for Configuration 1a scaled by values of $f_{F}>1$ and $f_{C}<1$, while varying the canard deflection angle. The overall effects on lift and drag with nonlinearities in angle-of-attack produces a maximum lift-to-drag around $f_{F}=1.95$ for $f_{C}=0.75$ and $\delta=4^{o}$. Figure 15 shows the results of the optimization routine at these conditions. Each plot features the trends in all 3 metrics, although the desire is to maximize on the lift-to-drag ratio at its trim angle of attack, as a function of a specific variable with all other variables held constant at the optimal value. For example, the top left plot shows the variation in trim angle, lift-to-drag, and normal force as a function of fin size with canard area scaled by 0.75 and the canard deflection angle set 
at $4^{\circ}$. The maximum lift-to-drag ratio of 3 occurs for a fin scaling factor of 1.95 although the variation is not large for the scaling factors shown. Reducing the size of the canards below 0.75 has a much greater effect on reducing the lift-to-drag ratio (top right plot of Fig. 15). Canard scaling factors above 0.75 are not shown as the projectile wasn't statically stable. The measures of performance are relatively flat with canard deflection angle due to canard flow separation effects.
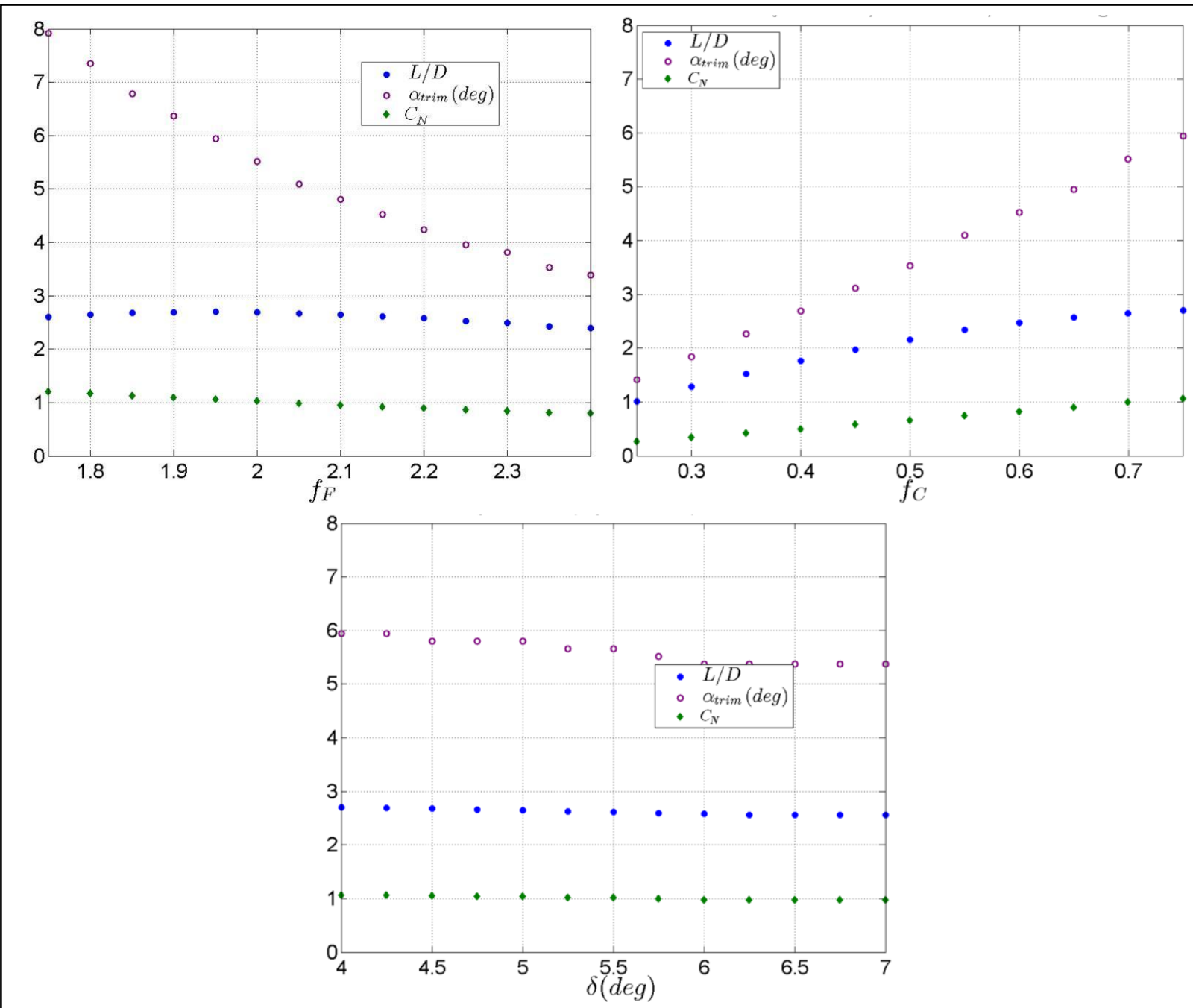

Fig. 15 Parameter sensitivity study using Configuration 1a aerodynamic data with $f_{F}=1.95, f_{c}=0.75$, and $\delta=4^{o}$

Figure 16 shows some optimization results using the aerodynamic coefficients obtained for Configuration $1 \mathrm{~b}$ scaled by $f_{F}=1.7$ and $f_{C}=0.75$ for $\delta=4^{\circ}$. Overall, the trends are consistent with the analysis for Configuration 1a in Fig. 15. Configuration $1 \mathrm{~b}$ has smaller canards, which means that, to get similar metrics as shown in Fig. 15 for Configuration 1b, the fin size does not need to be increased as much. The results compiled in Figs. 15 and 16 suggest that a larger total fin area (i.e., more and/or larger fins) is necessary. The exact amount will be determined after completing a comprehensive redesign to obtain an accurate $X_{c g}$ location. 


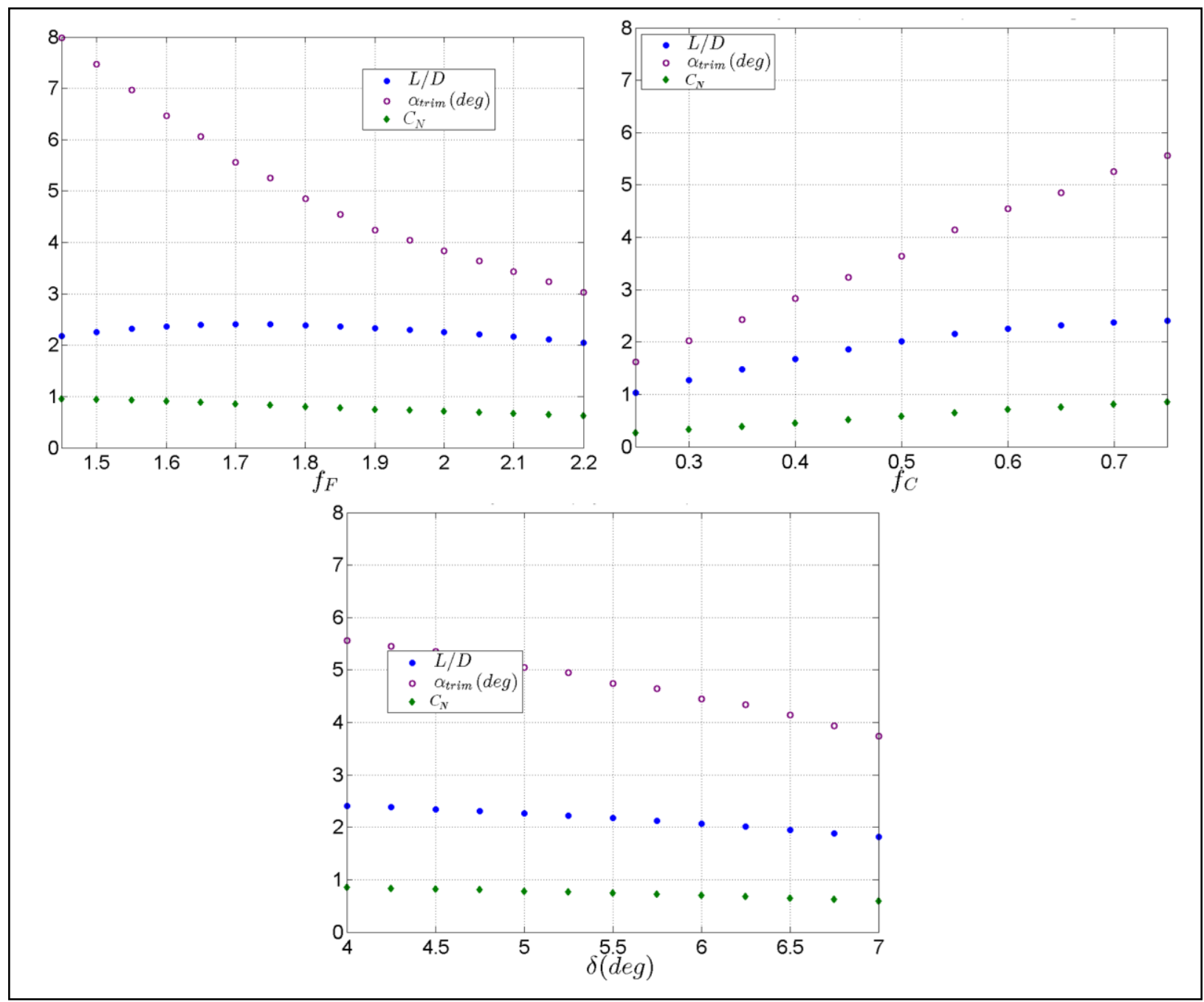

Fig. 16 Parameter sensitivity study using aerodynamic data from Configuration $1 \mathrm{~b}$ with $f_{F}=1.7, f_{c}=0.75$, and $\delta=4^{o}$

\subsection{Phase 2}

The main purpose of the second phase of this study was to validate that the optimized airframe design as determined in the first phase would meet the requirements to be used as the demonstration platform for the LCHAW mission program. The outcome of the first phase was an optimized airframe design that accounted for the interaction effects between the body, fins, and canards of the airframe. Prior to validating the aerodynamic performance of this design with additional CFD predictions, efforts were undertaken to further refine the design, such as better determination of mass properties, especially for the $X_{c g}$ location. Figure 17 shows the airframe components and their relative assumed placement, which were included in the solid model of the projectile to calculate mass properties. A number of other assumptions in designing the updated airframe configuration were made, including that the volume and mass of the potted electronics and batteries be fixed and that they be positioned directly behind the control actuation system (CAS) section, which was located directly behind the camera. It was assumed that the canards 
within the CAS were stowed folded forward. Finally, it was assumed that the fins were stowed folded forward and the fin hinge point was fixed. As such, the deployed fin location was also fixed.

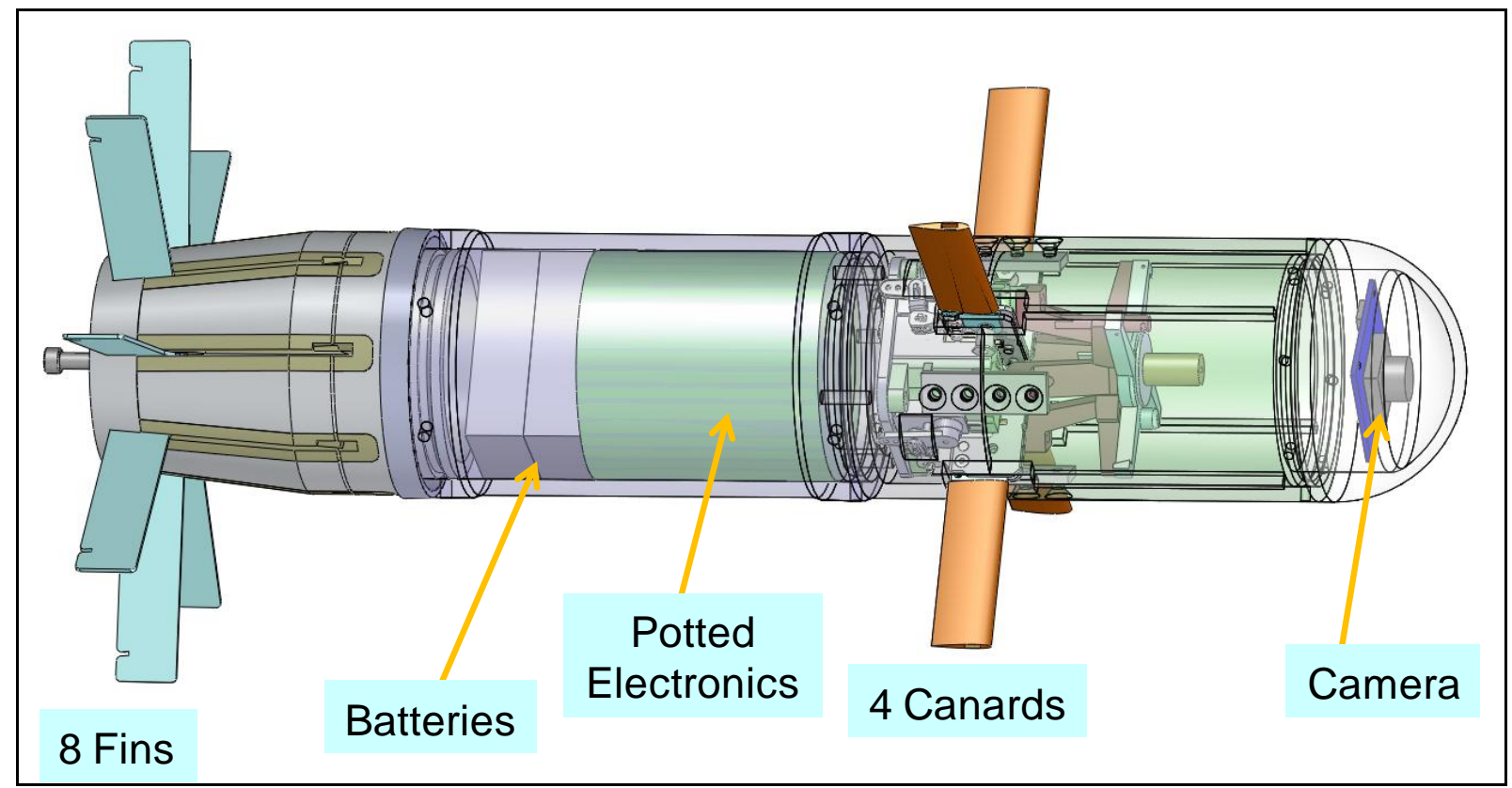

Fig. 17 Projectile components used to calculate mass properties

As with any design, there were a number of constraints that had to be met. The most limiting constraint was that the overall projectile length be fixed at $427.3 \mathrm{~mm}$. As the canards are assumed to fold forward within the CAS, the forward most canard pivot location needs to be constrained by the canard length (i.e., shorter canards can be positioned closer to the nose than long canards). Then, as fin length is adjusted for stability, a corresponding change in the length of the fin hub/boattail section needs to occur. Therefore, the rearmost limit of the guidance electronics section and the CAS section locations were adjusted by the same amount. This adjustment constrains the rearmost canard location. However, this constraint was not a limiting factor for any of the configurations that were evaluated because the guidance electronics never impinged on the fin hub. These constraints are illustrated in Fig. 18.

Using these additional geometric constraints, the parameter sensitivity study was completed with the aerodynamic coefficients of Configuration 1a and updated $X_{c g}$. By parameterizing fin area, canard area, and canard axis location, Configuration 2 was designed. 


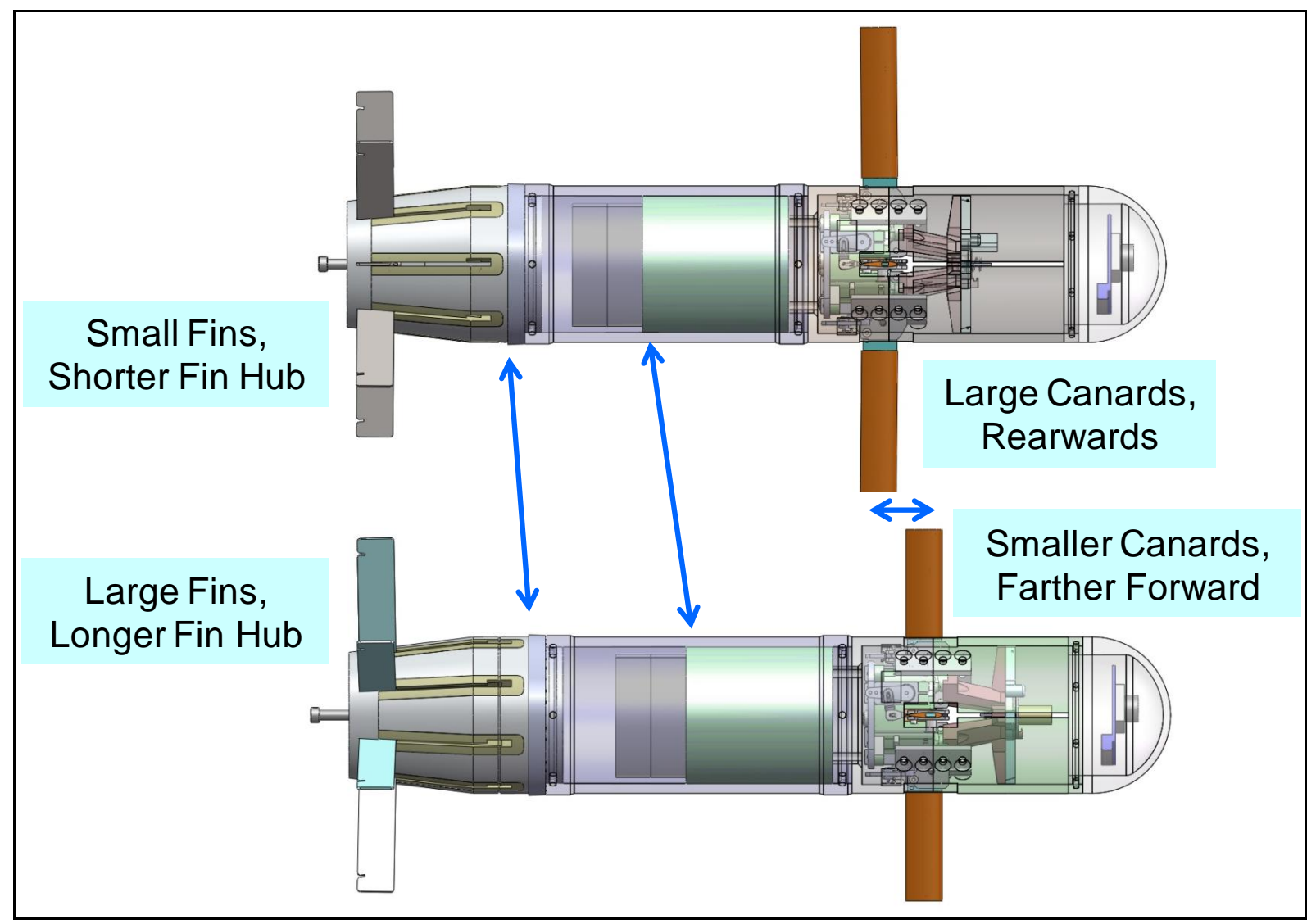

Fig. 18 Illustration of geometry constraints

Configuration 2 (Fig. 19) has 8 rectangular fins with a 199.87-mm tip-to-tip span, a 19.05-mm chord (200\% of Configuration 1a fin area), and a $1.27-\mathrm{mm}$ thickness with the trailing edge

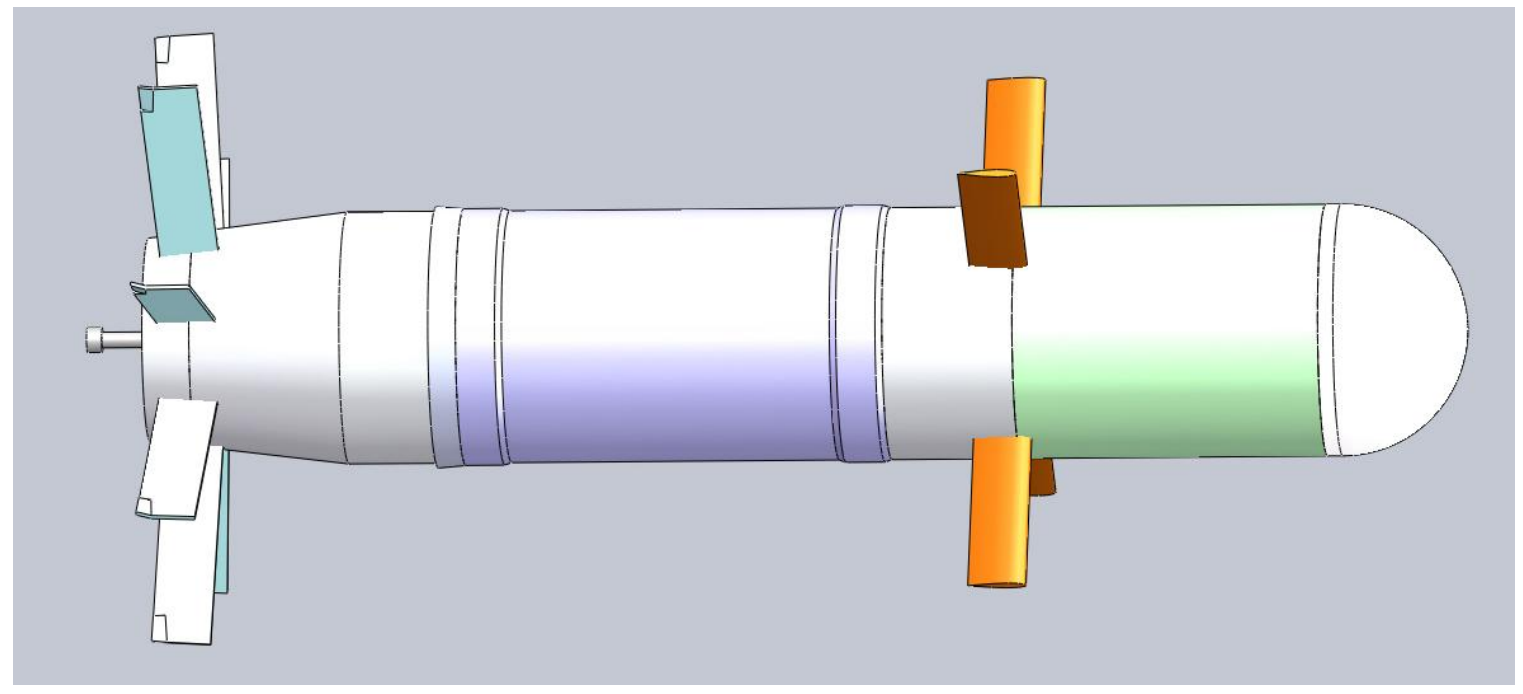

Fig. 19 Third body-fin-canard geometry (Configuration 2) 
located $5.10 \mathrm{~mm}$ from the base of the projectile. For ease of construction in the future, it was decided to use a bent fin tip to create roll torque rather than using a double bevel geometry that had been used in Configurations $1 \mathrm{a}$ and $1 \mathrm{~b}$. The top $8.79 \mathrm{~mm}$ of each fin of Configuration 2 was bent to an angle of $15^{\circ}$. Four NACA0015 airfoils in an " $\mathrm{X}$ " orientation (i.e., $45^{\circ}$ off plane) were used for the canards. The canard chord was $18.86 \mathrm{~mm}$ and the tip-to-tip span was $192.72 \mathrm{~mm}$ (70\% of Configuration 1a canard area). The canard axis (at the quarter-chord of the canard) was located $73.787-\mathrm{mm}\left(0.889 \mathrm{cal}\right.$.) forward of the $X_{c g}$. For the fin-canard offset, 2 fins were equally spaced between each set of canards for an alignment offset of $22.5^{\circ}$. Finally, to simulate a possible deployment mechanism for the fins in the future, a cavity was present in the base of the projectile along with a pin. The mass properties were calculated to be $X_{c g}=218 \mathrm{~mm}$ from the nose of the projectile, $I_{x}=0.0034 \mathrm{~kg}-\mathrm{m}^{2}$, and $I_{y}=0.0388 \mathrm{~kg}-\mathrm{m}^{2}$, and $m=2.65 \mathrm{~kg}$.

\subsubsection{Aerodynamic Characterization}

The static aerodynamic characterization of Configuration 2 was completed for only 1 Mach number, Mach 0.65 , as the change in mass properties indicated that the velocity would not vary significantly during its flight. Fourteen angles of attack, $-14^{\circ} \leq \alpha \leq 14^{\circ}$, were investigated. For each simulation, sufficient iterations were completed (typically 2000 to3000) to ensure convergence in both residual drop and aerodynamic coefficients. The aerodynamic coefficients were obtained by averaging their values over the last 200 iterations to minimize any oscillations (mostly present at higher angles of attack). The simulations were again completed on the IBM iDATAPlex system, Pershing, at the ARL DSRC. For this configuration, a mesh containing approximately 40.6 million cells was created. The 40.6 million cell mesh was decomposed using pMETIS to run on 272 processors ( 149,000 cells/processor), which resulted in an averaging processing time of approximately $9 \mathrm{~s}$ per iteration.

Only ballistic fits were required for the coefficient fits for the body and fin components with this configuration as inclusion of negative angles of attack here prove that $C_{X}$ and $C_{l}$ are in fact symmetric across $\alpha=0^{\circ}$. As such, the fit for $C_{X}$ reduces from Eqs. 16 to 21 and the fit for $C_{l}$ reduces from Eqs. 19 to 22 . The component contributions to the aerodynamic coefficients for the body and fins (total) are listed in Table 8 . The side force and moment contributions are currently considered to be negligible. However, this assumption is likely not to be the case if and when the canards are not all deflected equally. Thus, the body and total fin side force and moment contributions will have to be revisited as the design matures. Figure 20 shows how well these fits are able to predict the aerodynamic coefficients as determined by CFD. The fits for $C_{X}$ and $C_{l}$ predict the overall shape fairly well though the values near $\alpha=0^{\circ}$ are not as well predicted as they might be. At this point in the design process, the discrepancy in the predictions at $\alpha=0^{\circ}$ is not a concern. It will be investigated further in the future.

$$
\begin{gathered}
C_{X}=C_{X_{0}}+C_{X_{\alpha 2}} \delta^{2}+C_{X_{\alpha 4}} \delta^{4} \\
C_{l}=C_{l_{0}}+C_{l_{\alpha 2}} \delta^{2}+C_{l_{\alpha 4}} \delta^{4}
\end{gathered}
$$


Table 8 Static aerodynamic coefficient fits for Configuraion 2 at Mach 0.65 , body and fin components

\begin{tabular}{|c|c|c|}
\hline & Body & Fins \\
\cline { 2 - 3 }$C_{X_{0}}$ & 0.1538 & 0.1474 \\
\hline$C_{X_{\alpha 2}}$ & -0.1943 & 0.7101 \\
\hline$C_{X_{\alpha 4}}$ & 6.5204 & -4.8400 \\
\hline$C_{N_{\alpha}}$ & 3.7278 & 4.4948 \\
\hline$C_{N_{\alpha 3}}$ & -11.3042 & -25.6679 \\
\hline$C_{m_{\alpha}}$ & 3.9785 & -10.4992 \\
\hline$C_{m_{\alpha 3}}$ & -7.2300 & 59.3082 \\
\hline$C_{l_{0}}$ & $\ldots$ & 0.08242 \\
\hline$C_{l_{\alpha 2}}$ & $\ldots$ & 0.6111 \\
\hline$C_{l_{\alpha 4}}$ & $\ldots$ & -9.5560 \\
\hline
\end{tabular}



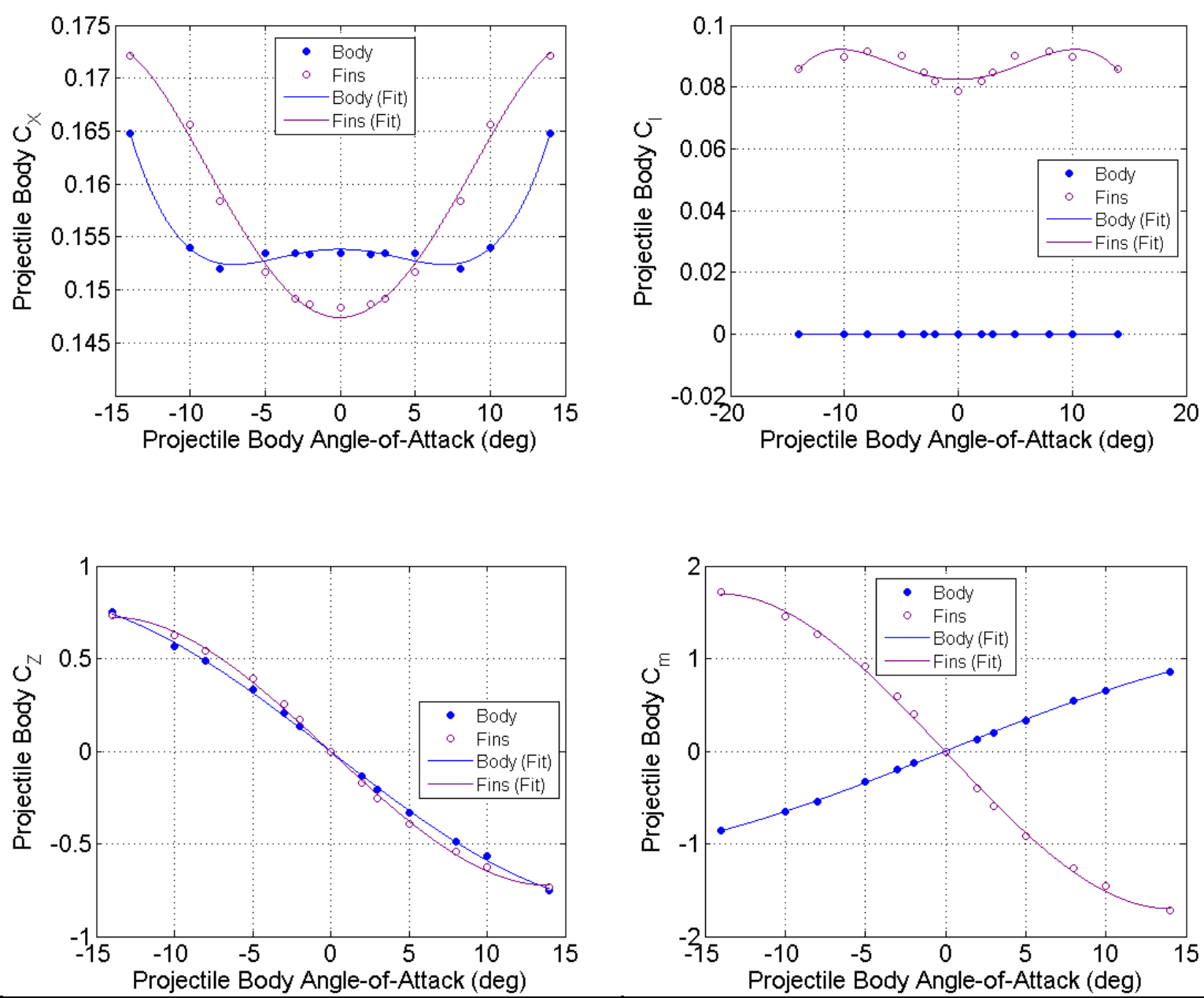

Fig. 20 Body and fin component fits for Configuration 2, Mach 0.65

Figure 21 shows the fits for 4 of the 6 static aerodynamic coefficients for the canards in the local blade coordinate systems; the side force and side moment are assumed to be 0 in the local blade coordinate system. Again, because the canard fits are in the local blade coordinate system, the side force and moment are assumed to be negligible. The numbering scheme for the canards remains the same as for the previous configuration (Fig. 4). For all the canard aerodynamic coefficients, it is quite apparent that the aerodynamic behavior changed at approximately $\alpha= \pm 5.5^{\circ} ; C_{Z}$ and $C_{m}$ were approximately constant when $|\alpha|>5.5^{\circ}$. Thus, it is likely that aerodynamic stall of the canards is occurring near this $\alpha$. As such, a trimodal fit $\left(\alpha>-5.5^{\circ}, \alpha>\right.$ $5.5^{\circ},-5.5^{\circ} \leq \alpha \leq 5.5^{\circ}$ ) was developed for the canard aerodynamic coefficients to best represent the data trends. To fit the data around $\alpha=0^{\circ}$, only ballistic fits were required for the individual canard components. As such, Eq. 21 is again used for $C_{X}$. However, $C_{l}$ for the canards is an odd fit (the constant term is still zero). This means that Eq. 19 reduces to Eq. 23 rather than Eq. 22. At the larger absolute angles of attack, after canard stall occurs, the coefficient fits, for all aerodynamic coefficients of interest, change drastically; $C_{X}$ is an odd fit (Eq. 24), while $C_{N}\left(-C_{Z}\right)$, 
$C_{m}$, and $C_{l}$ are linear fits (Eqs. 25 to 27 , respectively).To ensure that the transition between the segments of the trimodal fits is smooth, the value of the constant $\left(C_{X_{0}}, C_{N_{\alpha 0}}, C_{m_{\alpha 0}}, C_{l_{0}}\right.$, respectively) in the fits beyond stall is taken as the value of the coefficient at the stall angle, as appropriate.

$$
\begin{gathered}
C_{l}=C_{l_{\alpha 1}} \delta+C_{l_{\alpha 3}} \delta^{3} \\
C_{X}=C_{X_{0}}+C_{X_{\alpha 1}} \delta+C_{X_{\alpha 3}} \delta^{3} \\
C_{N}=C_{N_{\alpha 0}}+C_{N_{\alpha 1}} \delta \\
C_{m}=C_{m_{\alpha 0}}+C_{m_{\alpha 1}} \delta \\
C_{l}=C_{l_{0}}+C_{l_{\alpha 1}} \delta
\end{gathered}
$$

The component contributions of each of the 4 individual canards for each of the $\alpha$ ranges are listed in Tables 9 to 12 . After completing the fits, a more exact value of $\alpha= \pm 5.675^{\circ}$ was found to be the stall angle by setting the 2 fits equal to each other and solving for $\alpha$. 


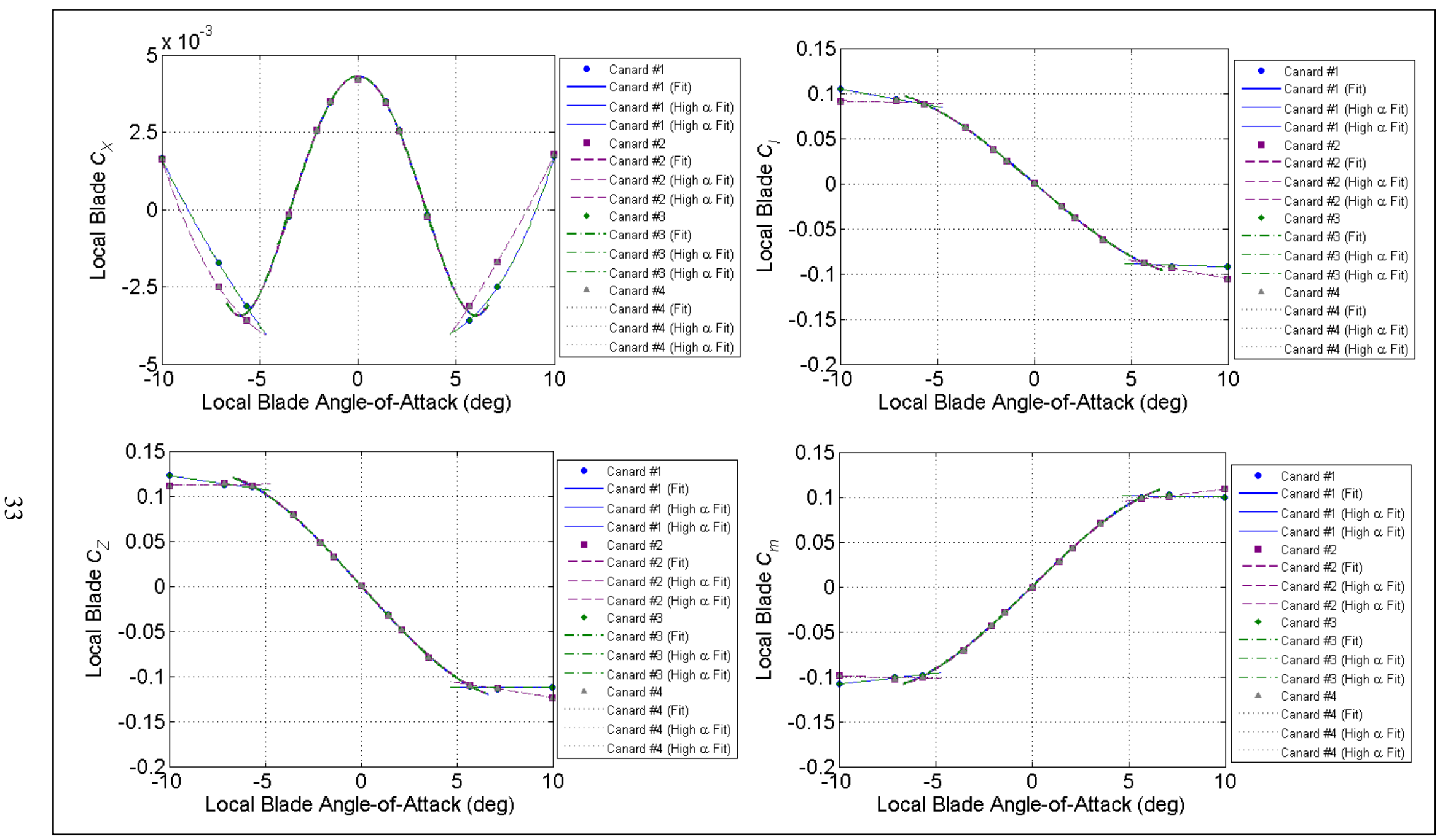

Fig. 21 Canard component fits in local blade coordinate system for Configuration 2, Mach 0.65. 
Table 9 Static aerodynamic coefficient fits for Canard 1 for Configuration 2, Mach 0.65

\begin{tabular}{|c|c|c|c|c|c|c|c|c|c|c|c|}
\hline$\alpha$ range & $C_{X_{0}}$ & $C_{X_{\alpha 1}}$ & $C_{X_{\alpha 2}}$ & $C_{X_{\alpha 3}}$ & $C_{X_{\alpha 4}}$ & $C_{N_{\alpha}}$ & $C_{N_{\alpha 3}}$ & $C_{m_{\alpha 1}}$ & $C_{m_{\alpha 3}}$ & $C_{l_{\alpha 1}}$ & $C_{l_{\alpha 3}}$ \\
\hline$\alpha<-5.675^{\circ}$ & $\ldots$ & -0.00911 & 0.0 & 1.399 & 0.0 & -0.00130 & 0.0 & -0.0160 & 0.0 & -0.0396 & 0.0 \\
\hline$-5.675^{\circ} \leq \alpha \leq 5.675^{\circ}$ & 0.00430 & 0.0 & -1.416 & 0.0 & 64.751 & 1.349 & -23.367 & 1.205 & -20.410 & -1.055 & 16.593 \\
\hline$\alpha>5.675^{\circ}$ & $\ldots$ & --0.0443 & 0.0 & -0.344 & 0.0 & 0.180 & 0.0 & 0.139 & 0.0 & -0.223 & 0.0 \\
\hline
\end{tabular}

Table 10 Static aerodynamic coefficient fits for Canard 2 for Configuration, Mach 0.65

\begin{tabular}{|c|c|c|c|c|c|c|c|c|c|c|c|}
\hline$\alpha$ range & $C_{X_{0}}$ & $C_{X_{\alpha 1}}$ & $C_{X_{\alpha 2}}$ & $C_{X_{\alpha 3}}$ & $C_{X_{\alpha 4}}$ & $C_{N_{\alpha}}$ & $C_{N_{\alpha 3}}$ & $C_{m_{\alpha 1}}$ & $C_{m_{\alpha 3}}$ & $C_{l_{\alpha 1}}$ & $C_{l_{\alpha 3}}$ \\
\hline$\alpha<-5.675^{\circ}$ & & 0.0440 & 0.0 & 0.378 & 0.0 & 0.191 & 0.0 & 0.149 & 0.0 & -0.232 & 0.0 \\
\hline$-5.675^{\circ} \leq \alpha \leq 5.675^{\circ}$ & 0.00430 & 0.0 & -1.417 & 0.0 & 64.802 & 1.349 & - & 1.204 & -20.315 & -1.055 & 16.522 \\
\hline$\alpha>5.675^{\circ}$ & $\ldots$ & 0.00396 & 0.0 & -1.297 & 0.0 & -0.0110 & - & -0.238 & 0.0 & -0.0321 & 0.0 \\
\hline
\end{tabular}

Table 11 Static aerodynamic coefficient fits for Canard 3 for Configuration 2, Mach 0.65

\begin{tabular}{|c|c|c|c|c|c|c|c|c|c|c|c|}
\hline$\alpha$ range & $C_{X_{0}}$ & $C_{X_{\alpha 1}}$ & $C_{X_{\alpha 2}}$ & $C_{X_{\alpha 3}}$ & $C_{X_{\alpha 4}}$ & $C_{N_{\alpha}}$ & $C_{N_{\alpha 3}}$ & $C_{m_{\alpha 1}}$ & $C_{m_{\alpha 3}}$ & $C_{l_{\alpha 1}}$ & $C_{l_{\alpha 3}}$ \\
\hline$\alpha<-5.675^{\circ}$ & 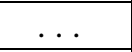 & -0.00886 & 0.0 & 1.395 & 0.0 & -0.00126 & 0.0 & -0.0159 & 0.0 & -0.0396 & 0.0 \\
\hline$-5.675^{\circ} \leq \alpha \leq 5.675^{\circ}$ & 0.00430 & 0.0 & -1.416 & 0.0 & 64.760 & 1.349 & -23.374 & 1.205 & -20.416 & -1.055 & 16.597 \\
\hline$\alpha>5.675^{\circ}$ & $\ldots$ & -0.0441 & 0.0 & -0.349 & 0.0 & 0.180 & 0.0 & 0.139 & 0.0 & -0.223 & 0.0 \\
\hline
\end{tabular}

Table 12 Static aerodynamic coefficient fits for Canard 4 for Configuration 2, Mach 0.65

\begin{tabular}{|c|c|c|c|c|c|c|c|c|c|c|c|}
\hline$\alpha$ range & $C_{X_{0}}$ & $C_{X_{\alpha 1}}$ & $C_{X_{\alpha 2}}$ & $C_{X_{\alpha 3}}$ & $C_{X_{\alpha 4}}$ & $C_{N_{\alpha}}$ & $C_{N_{\alpha 3}}$ & $C_{m_{\alpha 1}}$ & $C_{m_{\alpha 3}}$ & $C_{l_{\alpha 1}}$ & $C_{l_{\alpha 3}}$ \\
\hline$\alpha<-5.675^{\circ}$ & $\ldots$ & 0.0440 & 0.0 & 0.378 & 0.0 & 0.191 & 0.0 & 0.148 & 0.0 & -0.232 & 0.0 \\
\hline$-5.675^{\circ} \leq \alpha \leq 5.675^{\circ}$ & 0.00430 & 0.0 & -1.417 & 0.0 & 64.791 & 1.349 & -23.252 & 1.204 & -20.311 & -1.055 & 16.520 \\
\hline$\alpha>5.675^{\circ}$ & $\ldots$ & 0.00385 & 0.0 & -1.295 & 0.0 & -0.011 & 0.0 & -0.0239 & 0.0 & -0.0321 & 0.0 \\
\hline
\end{tabular}


Having completed the component fits, the stability of the projectile and the canard stall angle can be investigated. With no canard deflection (Fig. 22), the total force and pitching moment should, and do, match the total values as determined by CFD. The center-of pressure location is predicted to be just under 0.5 calibers behind the $X_{c g}$ for angles of attack, indicating the current design is indeed stable. Additionally, canard stall occurs at approximately $\alpha= \pm 8^{\circ}$ (projectile body angle), where the magnitude of the canard contribution to these coefficients becomes approximately constant.
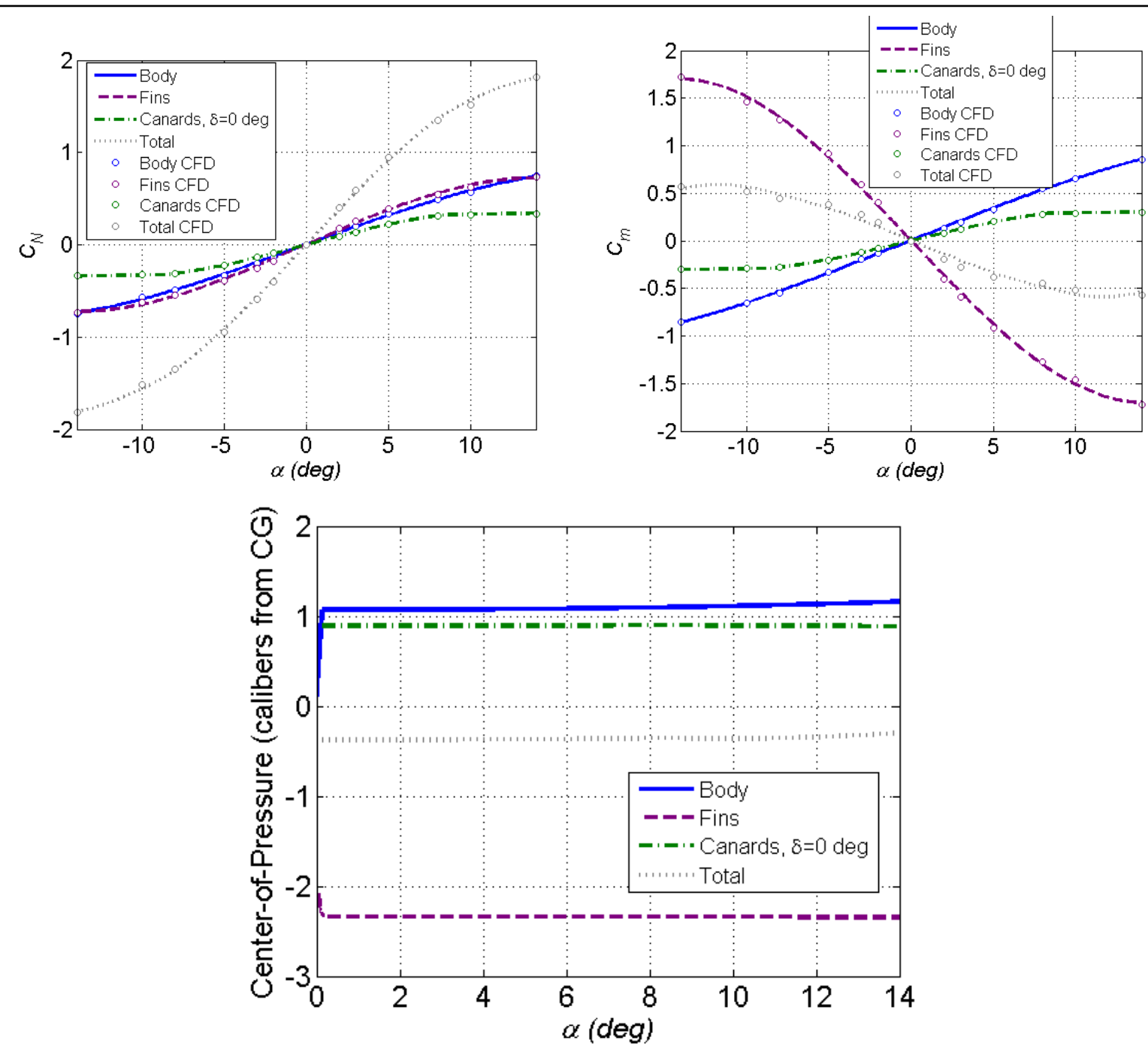

Fig. 22 Normal force, pitching moment, and center-of-pressure location build ups using CFD component fits with $0^{\circ}$ canard deflection for Configuration 2, Mach 0.65

In addition, the accuracy of assuming that the interaction effects of the canards on the fins scales with the fins (i.e., lumping the interaction effects into the fin component), as was done in the first phase, can be determined. The aerodynamic coefficients determined from the CFD simulation of Configuration 2 (Fig. 22) are compared to those predicted when the scaling factors representative 
of Configuration $2\left(f_{F}=2.0, f_{C}=0.7, x_{C}=0.88, X_{c g}=2.63\right)$ were applied to Configuration 1a aerodynamic data (Fig. 23). The normal force and pitching moment of the body component (Fig. 24) vary between the CFD solution and the predictions by less than $10 \%$. The fin component (Fig. 25) predictions under predict the CFD solution by $25 \%$ for $C_{N}$ and $15 \%$ for $C_{m}$. These significantly smaller variations indicate that combining the interaction effects with the contribution is a viable option during the initial design process.
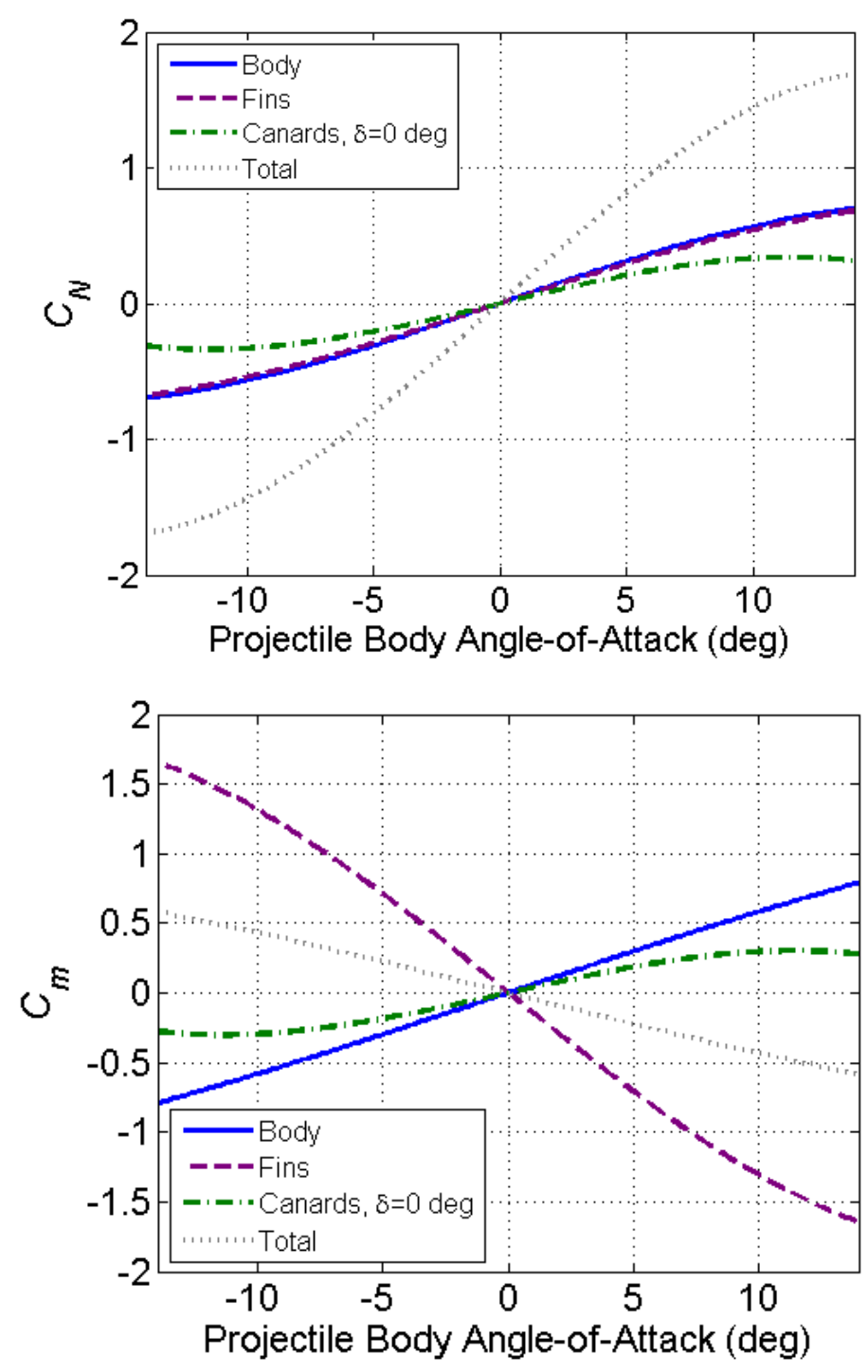

Fig. 23 Component build up of normal force and pitching moment using scaled component contributions $\left(f_{F}=2.0, f_{c}=0.7, x_{c}=0.88, X_{c g}=2.63\right)$ from CFD analysis of Configuration 1a to obtain theoretical values for Configuration 2, Mach 0.65

Scaling the interaction effects caused the fin effectiveness to be under predicted whereas neglecting it caused it to be over predicted. The under prediction is preferable as it is easy to make a projectile less stable. Being able to scale the interaction effects will limit the number of 
simulations and grids that are required during the design process as determination of interaction effects would require additional simulations on body-alone, body-fin, and body-canard geometries. The differences in the normal force and pitching moment of the canard component (Fig. 26) appear to be due entirely to the occurrence of canard stall in Configuration 2. Perhaps the presence of the additional fins ( 8 on Configuration 2 vs. 4 on Configurations 1a and 1b) at subsonic velocities caused feedback upstream to the canards.
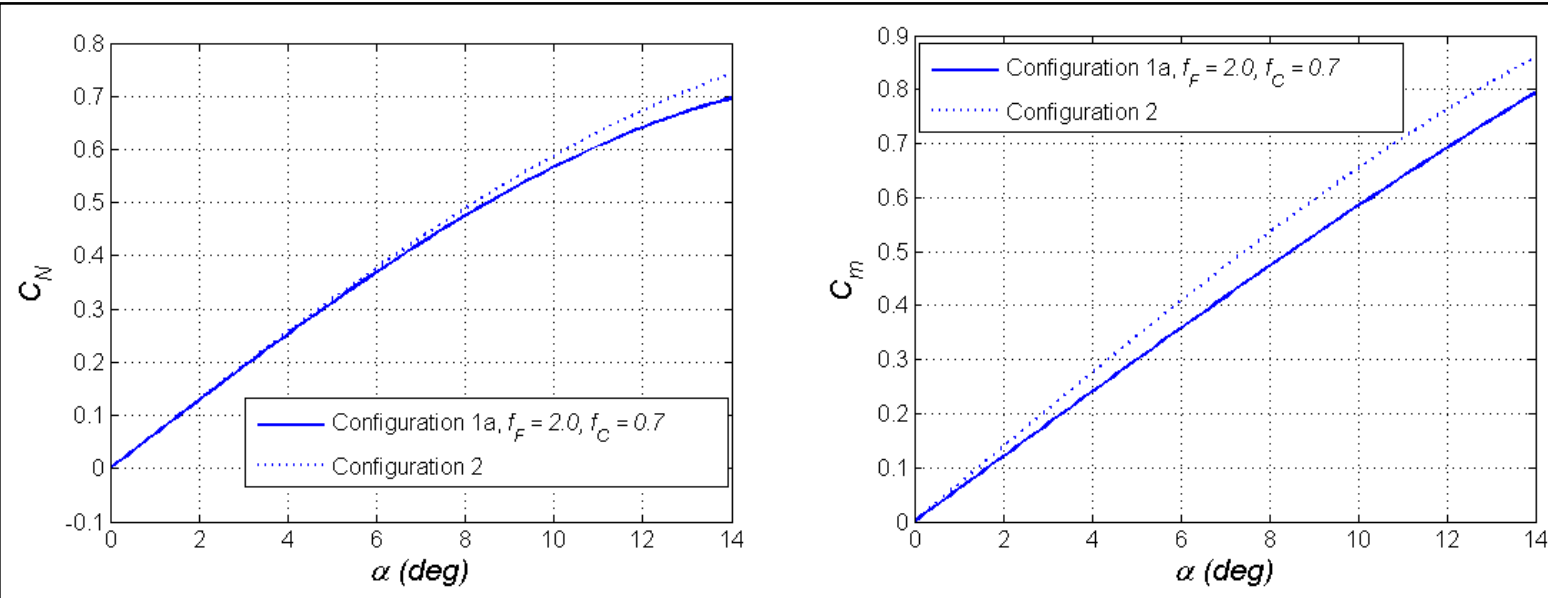

Fig. 24 Comparison of body component for Configuration 2 as determined from component buildup using Configuration 1a aerodynamics with form factors and CFD simulations, Mach 0.65
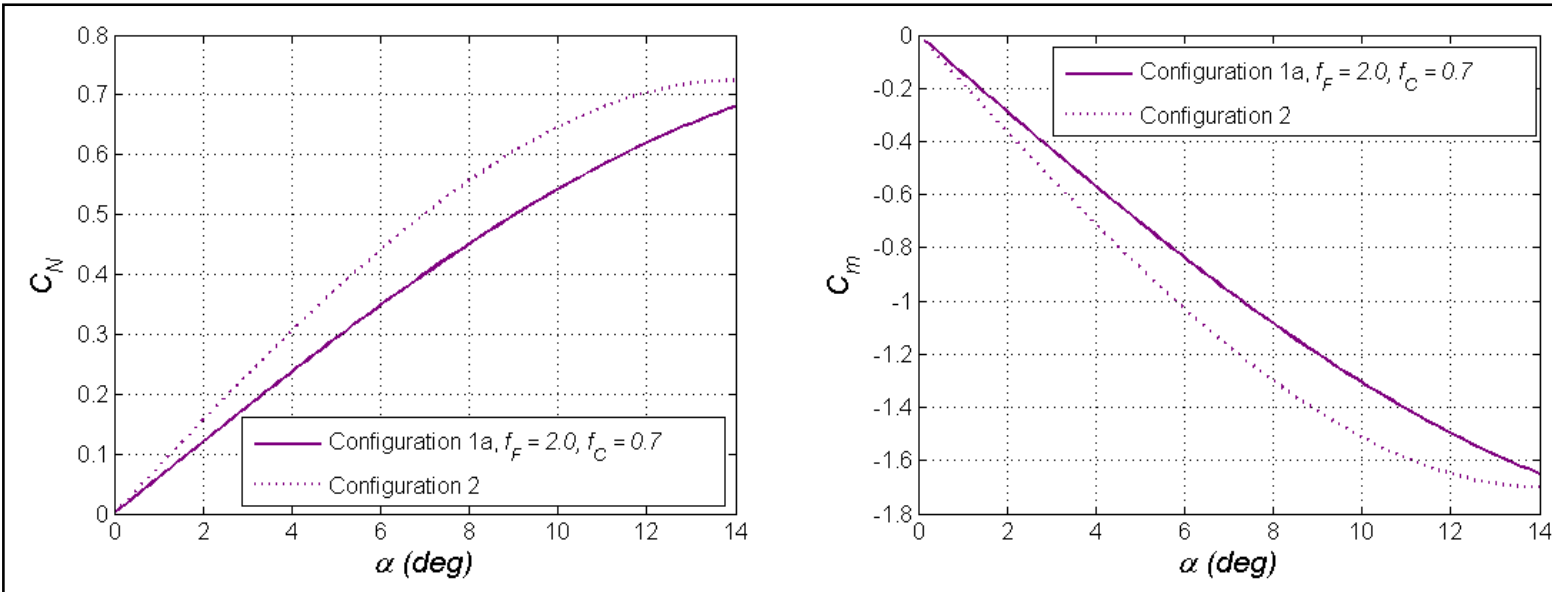

Fig. 25 Comparison of fin component for Configuration 2 as determined from component buildup using Configuration 1a aerodynamics with form factors and CFD simulations, Mach 0.65 

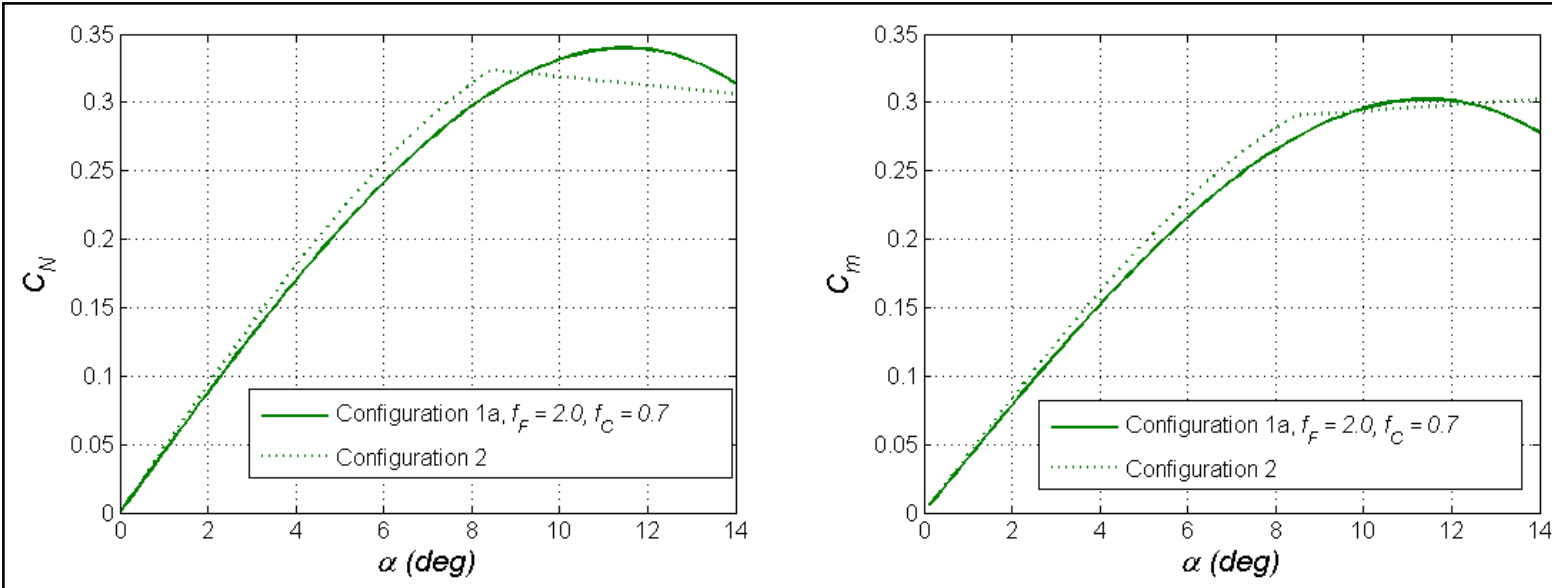

Fig. 26 Comparison of canard component for Configuration 2 as determined from component buildup using Configuration 1a aerodynamics with form factors and CFD simulations, Mach 0.65

Knowing the effect of canard deflection on the stability of the projectile was also desired. Instead of completing additional simulations with canard deflection, the effect of canard deflection was once again estimated by using total canard angle of attack $(\delta+\alpha)$ to determine the canard component contribution to the aerodynamics of the airframe. When a canard deflection angle of $\delta=4^{\circ}$ is investigated (Fig. 27), canard stall is predicted to occur at approximately $\alpha=3^{\circ}-4^{\circ}$ body angle as would be expected. As such, total $C_{m}$ doesn't continue to decrease as rapidly, nor does total $C_{N}$ increase as rapidly. Examining the $C_{m}$ and center-of-pressure plots in Fig. 27, the Configuration 2 airframe reaches a trimmed state at approximately $\alpha=3^{\circ}$. The presence of canard stall appears to cause a maximum trim angle of $\alpha=3^{\circ}$ even as the deflection angle further increases to $\delta=6^{\circ}$ (Fig. 28). The actual variation of canard stall angle with canard deflection will need to be investigated in the future to determine what the maximum trim angle could be. 

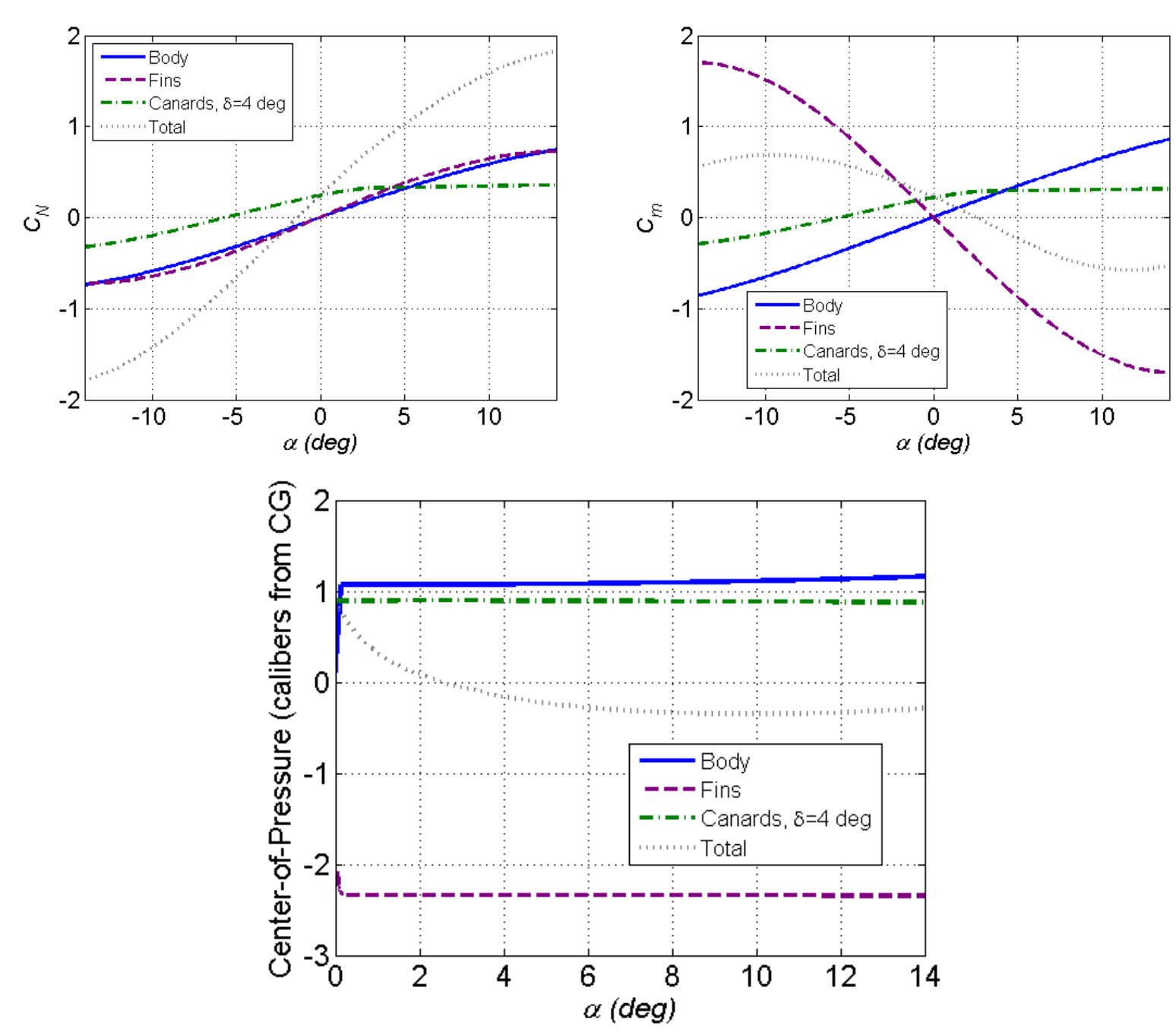

Fig. 27 Normal force, pitching moment, and center-of-pressure location build ups using CFD component fits with $4^{\circ}$ canard deflection for Configuration 2, Mach 0.65 

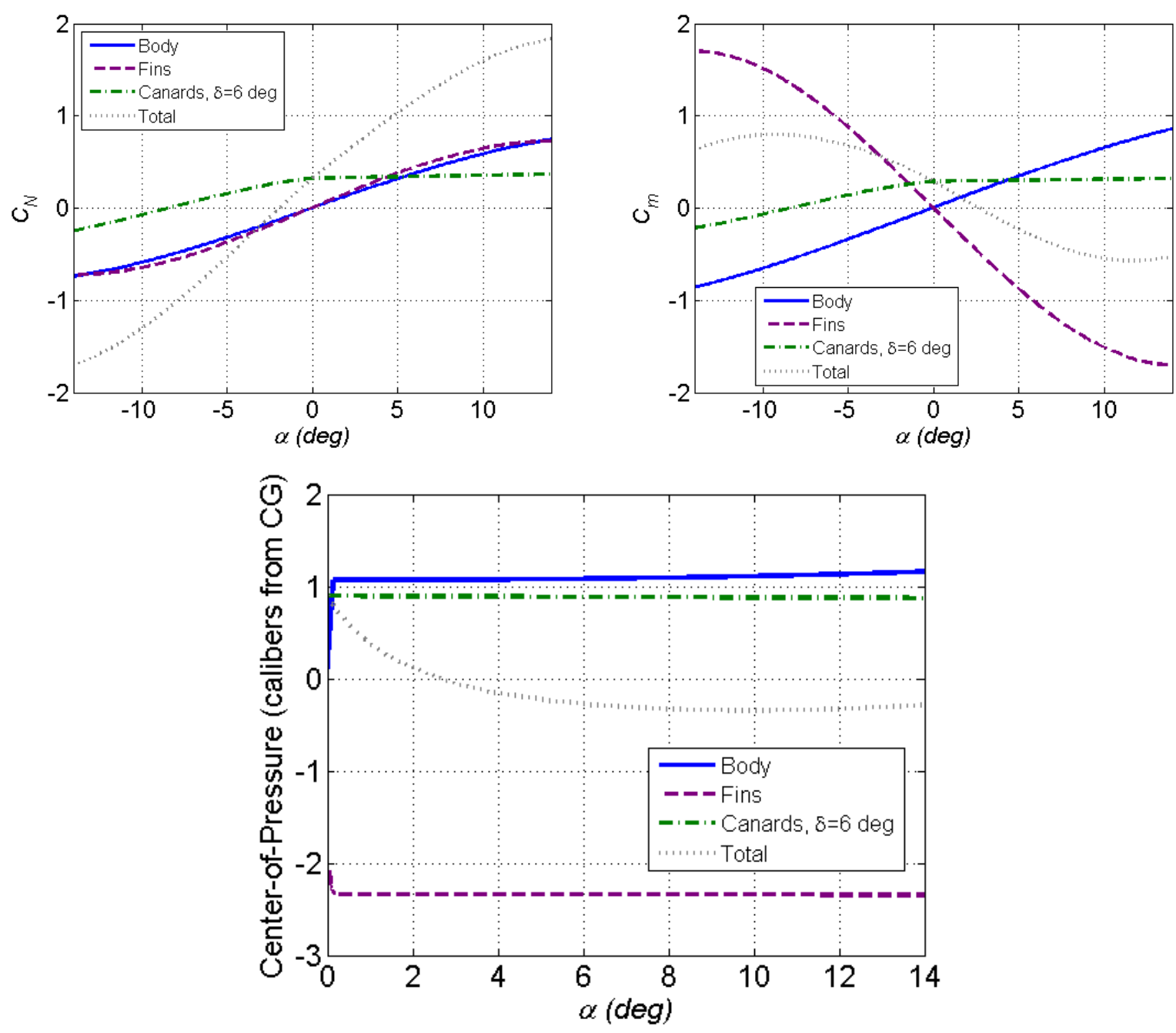

Fig. 28 Normal force, pitching moment, and center-of-pressure location build ups using CFD component fits with $6^{\circ}$ canard deflection for Configuration 2, Mach 0.65

As Configuration 2 was determined to be stable for small to moderate canard deflection angles, it is likely to be the platform of choice as the program moves forward. Therefore, additional knowledge of the design, such as equilibrium roll rate, was desired so that necessary modifications to the design could be made prior to moving forward. The equilibrium roll rate of the current design is a balance of the roll torque being generated by the bend of the fin tips and the roll damping generated by the body as well as the size of the fins and canards. The roll torque coefficient at $\alpha=0^{\circ}$ was determined from the static computation. However, a separate simulation, a rotating reference frame computation using the technique in DeSpirito et al, ${ }^{15}$ was required to determine the roll damping coefficient. From this computation, the roll damping coefficient, $C_{l_{p}}$, for the complete configuration (body-tail-canards) was determined to be -10.392 . 
Equation 28 was used to predict the equilibrium roll rate, $p_{e q}$, of $6.34 \mathrm{~Hz}$. While this roll rate is about double the desired roll rate, it remains a good place to start.

$$
p_{e q}=-\frac{C_{l}}{C_{l_{p}}} \frac{2 \mathrm{~V}}{D}
$$

While the primary purpose of this investigation was to design a stable platform for the LCHAW moving target mission program, another research goal was to begin to better understand the interaction of the canard tip vortices with the fins. Figure 29 shows how the streamlines that begin at the canards with $\delta=0^{\circ}$ change as the angle of attack increases from $\alpha=0^{\circ}$ to $\alpha=14^{\circ}$. For $\delta=0^{\circ}$ and $\alpha=0^{\circ}$, there is a complete lack of canard tip vortices and interference with the fins. The lack of tip vortices and interference continues at small angles of attack. As $\alpha$ increases to $3^{\circ}$, canard tip vortices are starting to develop. It is not until $\alpha$ increases beyond $5^{\circ}$ that the vortices appear to twist enough to interfere with the fins. Interference is minimal at $\alpha=5^{\circ}$, but is occurring by $\alpha=8^{\circ}$. Figure 30 shows the $\alpha=10^{\circ}$ case from the rear of the projectile.

The necessity of accounting for the interaction effects for all 4 canards becomes quickly apparent. Obviously, not all of the fins are affected, especially those fins on the windward side of the projectile. However, only some of the fins are affected by the tip vortices; the tip vortices of the top canards are swept away from the fins when the projectile is pitched up. The streamlines from inboard of all 4 canards appear to affect both the body and the fins. The exact effects will have to be investigated further, especially the effects when the canards are deflected. 


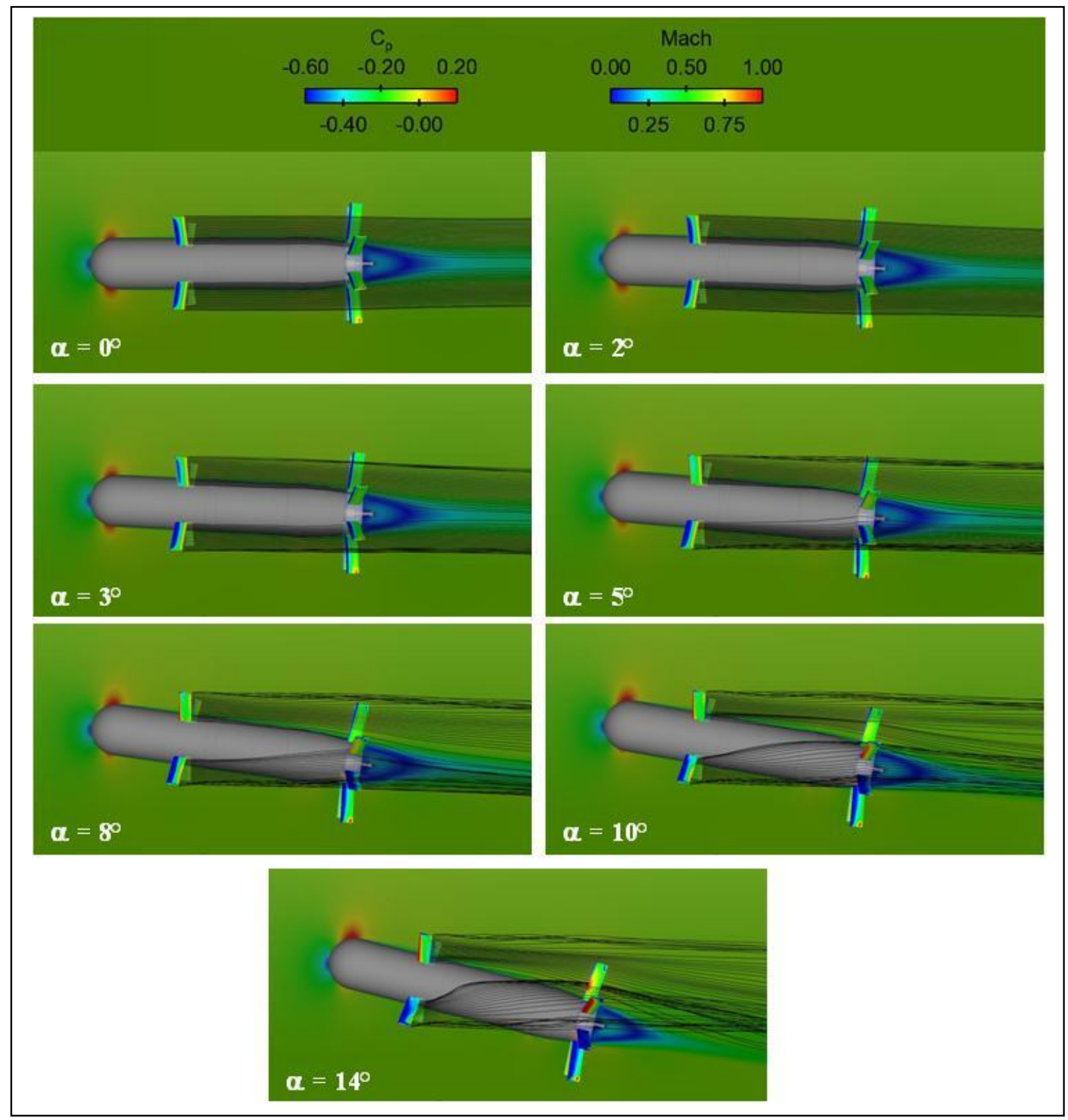

Fig. 29 Pitch plane Mach number contours with pressure coefficient contours on fins and canards $\left(\delta=0^{\circ}\right)$ with streamlines at various angles of attack for Configuration 2, Mach 0.65 


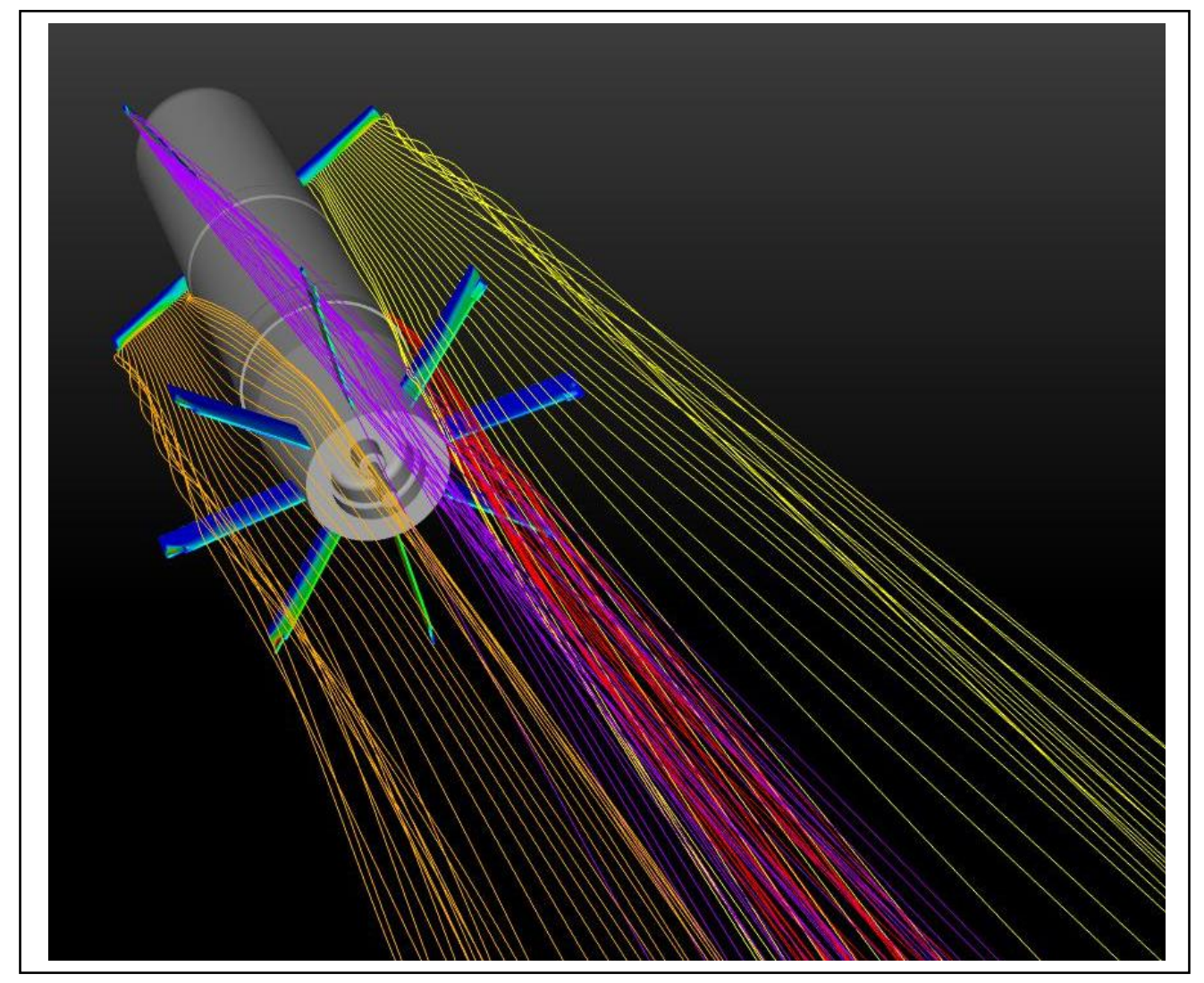

Fig. 30 Individual canard streamlines, rear view, for Configuration 2, Mach 0.65

\subsubsection{Parameter Sensitivity Study}

Because there were differences between the predicted and actual aerodynamic performance of the round, it was decided to use the parameter sensitivity routine to determine if any further modifications to the geometry could provide additional maneuverability through an increase to the lift-to drag ratio. Thus, the aerodynamic data for Configuration 2 were used as the input for the optimization and the parameters of interest (fin and canard area through $f_{F}$ and $f_{C}$ ) varied. Unfortunately, canard axis location was not shifted as the canard area (i.e., span as the chord is fixed due to geometric constraints) was varied, which may not provide for ideal results. These results are provided in Fig. 31 for Configuration 2 with a reduction in fin area of $20 \%\left(f_{F}=0.8\right)$, an increase in canard area of $50 \%\left(f_{C}=1.5\right)$, and $\delta=4^{\circ}$. This specific configuration, which is likely marginally stable, provides a maximum lift-to-drag ratio around 3, and produces a trim angle of approximately $6^{\circ}$. The trends with fin size, canard size, and canard deflection are similar to those for Configurations $1 \mathrm{a}$ and $1 \mathrm{~b}$. These data indicate that some additional fin area reduction and canard area addition may enhance maneuverability (i.e., more normal force, larger trim angle, etc.). However, some margin is warranted to account for uncertainty. Thus, Configuration 2 is chosen as the airframe for further consideration. 


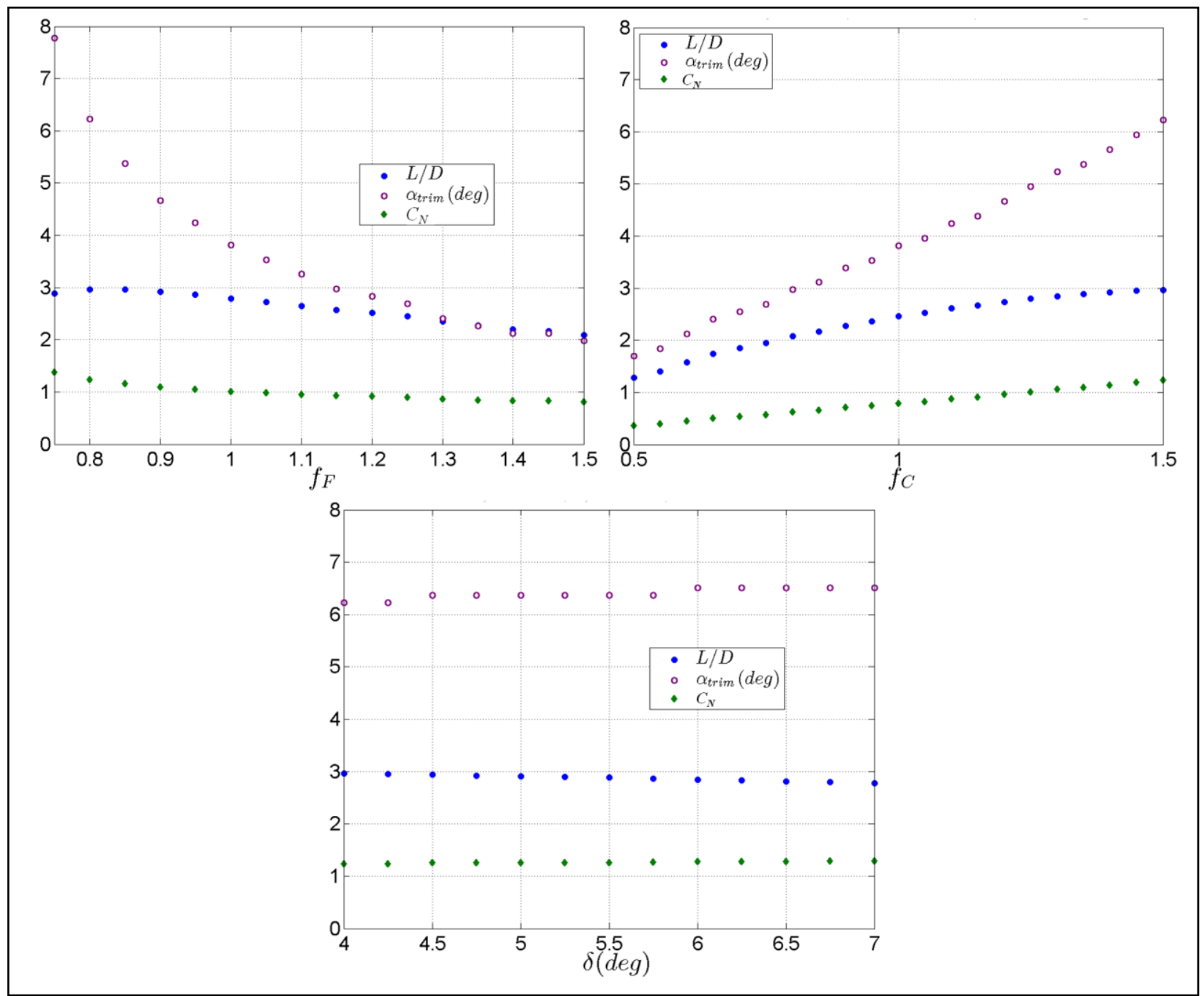

Fig. 31 Optimization analysis using Configuration 2 aerodynamic data with $f_{F}=0.8, f_{c}=1.5$, and $\delta=4^{\circ}$

\subsubsection{Linear Flight Mechanics and Control}

A detailed study of the controlled flight performance was undertaken based on the favorable aerodynamic characteristics of Configuration 2. The linear flight mechanics were implemented in simulation and driven with the appropriate aerodynamic, mass property, and actuator data. The flight control commands canard deflection to achieve a desired lateral acceleration while minimizing angular rate.

Some linear simulation results in the pitch plane for Configuration 2 are provided in Fig. 32. The top left plot shows that the control sufficiently reduces the pitch rate to the reference pitch rate $\left(q_{r}\right)$ of zero. The measured pitch rate $\left(q_{M}\right)$ and actual pitch rate $(q)$ are equivalent since perfect feedback was used in the simulations. A reference pitch acceleration $\left(\dot{w}_{r}\right)$ of $40 \mathrm{~m} / \mathrm{s}^{2}$ was desired and the response (measured pitch acceleration $\left(\dot{w}_{M}\right)$ and actual pitch acceleration $(\dot{w})$ equivalent since perfect feedback) suggests good performance since the rise time is less than $0.1 \mathrm{~s}$. The 
commanded and actual pitch deflections ( $\delta_{q_{c}}$ and $\delta_{q}$, respectively) to achieve these pitch rate and pitch acceleration responses are also shown in Fig. 32. Approximately $9^{\circ}$ of deflection yielded $40 \mathrm{~m} / \mathrm{s}^{2}$ of acceleration.

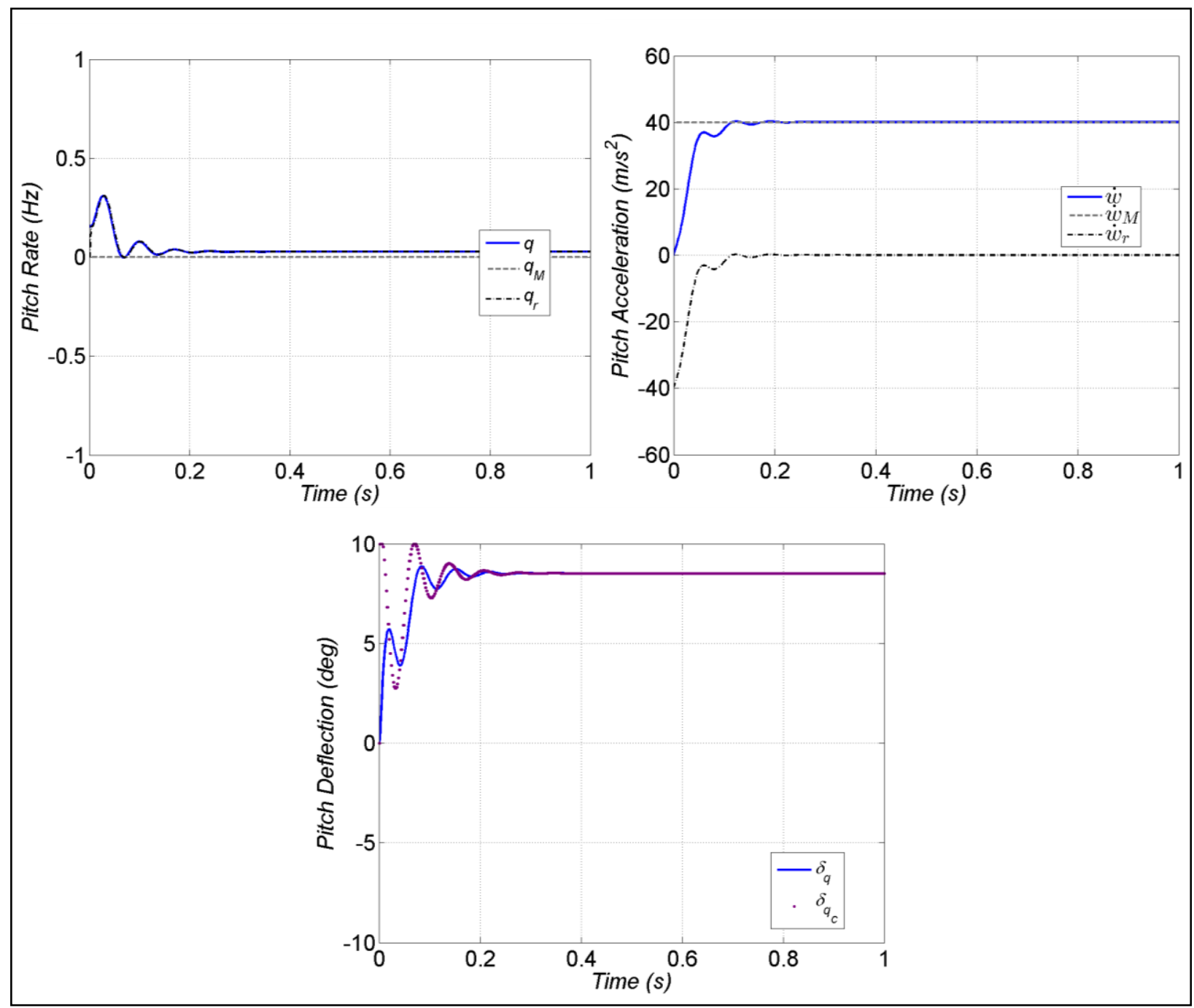

Fig. 32 Response and commands in pitch from linear modeling and simulation

\subsubsection{Nonlinear Flight Mechanics and Control}

Simulations of the controlled flight were performed on Configuration 2 using the nonlinear flight mechanics models. A target was placed approximately $1000 \mathrm{~m}$ downrange moving about $5 \mathrm{~m} / \mathrm{s}$ in the crossrange direction. Uncertainties or variations were placed on the target characteristics, launch, and projectile technologies to account for real-world effects. Uncompensated launch error sources (e.g., muzzle velocity variation, gun point angle, and initial angular disturbances) were modeled. The roll angle, angular rate sensor, accelerometer, and line-of-sight rate feedback were corrupted. Nominal actuator characteristics, projectile mass properties, and aerodynamics were perturbed. Monte Carlo techniques were applied in the simulations. 
The trajectory and aerodynamic angle histories for a representative flight are presented in Fig. 33. The guided projectile is the solid blue trace and the target is the dashed red line in the trajectory plots. The projectile moves about $30 \mathrm{~m}$ off the line-of-fire in the crossrange and altitude directions due to maneuvers necessary to intercept the target and the action of gravity. These data indicate a successful moving target intercept with small point-of-closest-approach. The maneuvers required for this engagement are well within the bounds of the Configuration 2 airframe since the pitch and yaw angles-of-attack are less than $1^{\circ}$ throughout this flight.

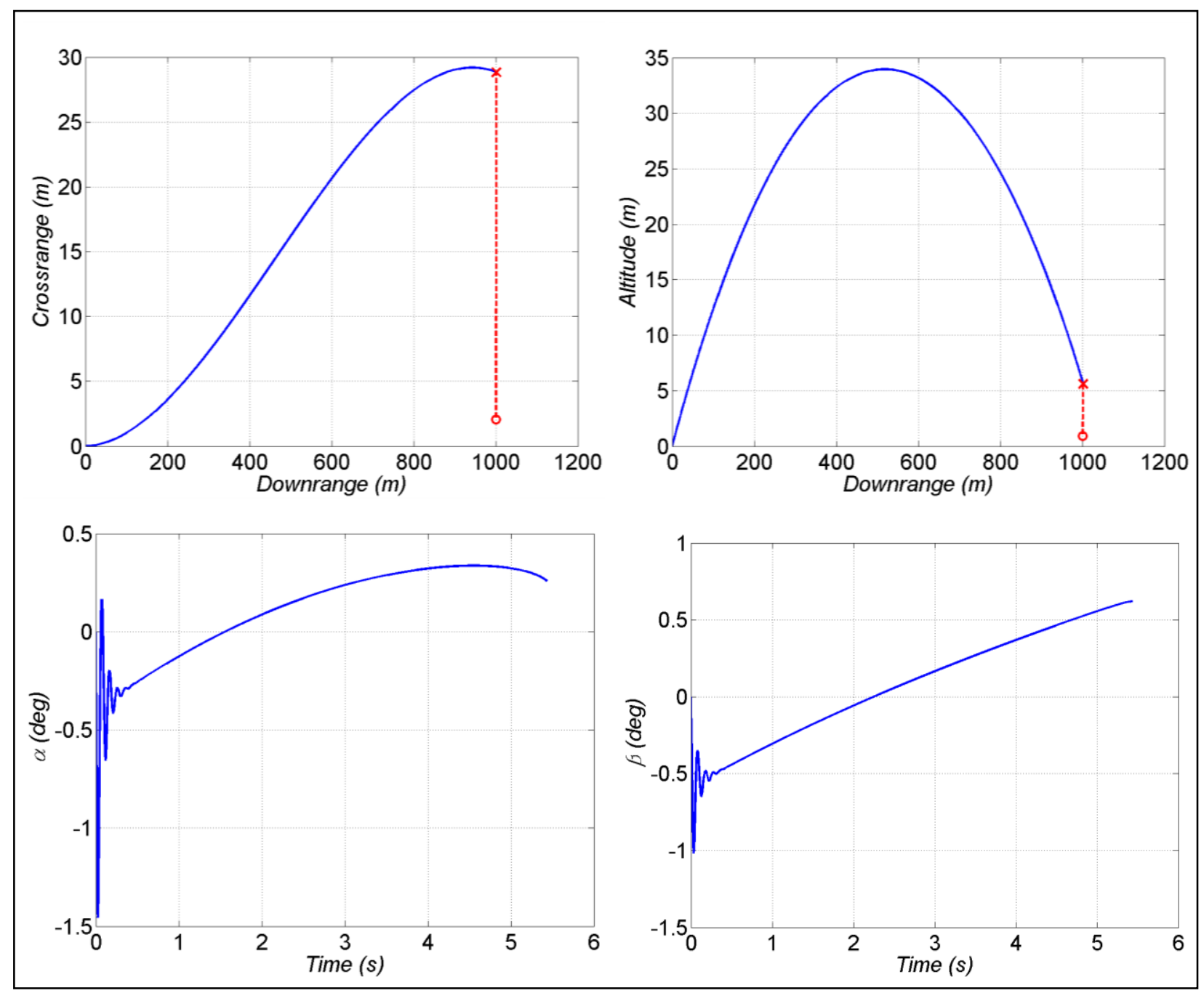

Fig. 33 Trajectory and aerodynamic angle histories for representative flight of Configuration 2

The objective of the flight control is to track specific roll angles, null out angular rates, and achieve desired lateral accelerations. The performance of the controller for Configuration 2 using the nonlinear models for this representative flight is provided in Fig. 34. Each plot features the true value, the measured (corrupted truth) value, and, for all plots except roll angle, the desired value. The roll angle is controlled to within $10^{\circ}$ of the reference point. Inspection of all angular 
rate histories (roll, pitch, and yaw) clearly shows good performance because rates are near zero for the majority of the flight. Pitch and yaw acceleration tracking is satisfactory. Measurement error is evident in all plots, although specifically called out for the roll angle.
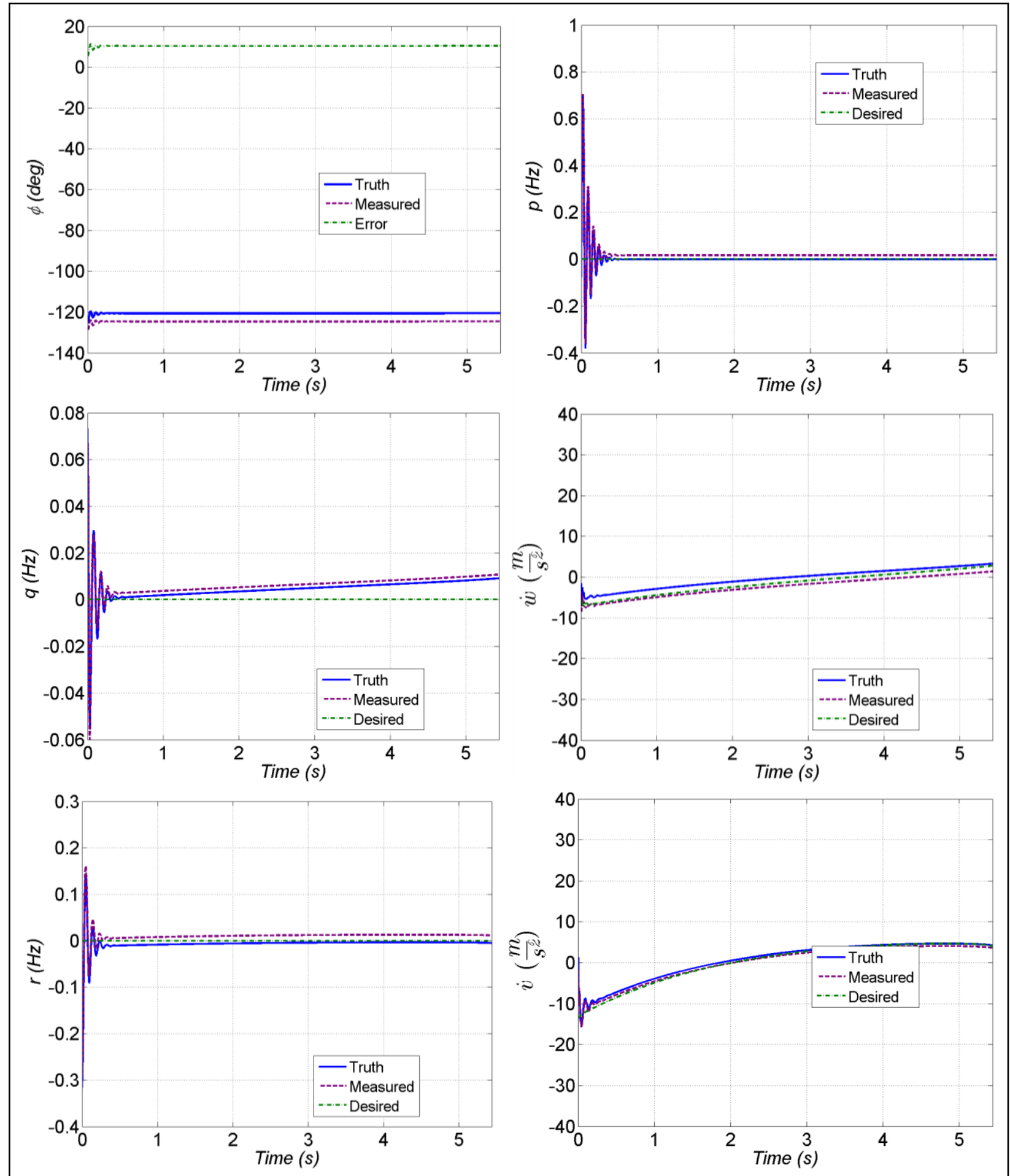

Fig. 34 Controlled response histories in roll, pitch, and yaw for representative flight of Configuration 2 
The individual canard histories from this representative flight are given in Fig. 35. The commands are shown along with the true value. A first-order model with a bias causes the command and actual values to differ. Some action is evident early in the flight to initially control the roll angle and angular rates. Only small variation is required throughout the majority of the flight to meet the lateral acceleration requirements for intercepting the target.
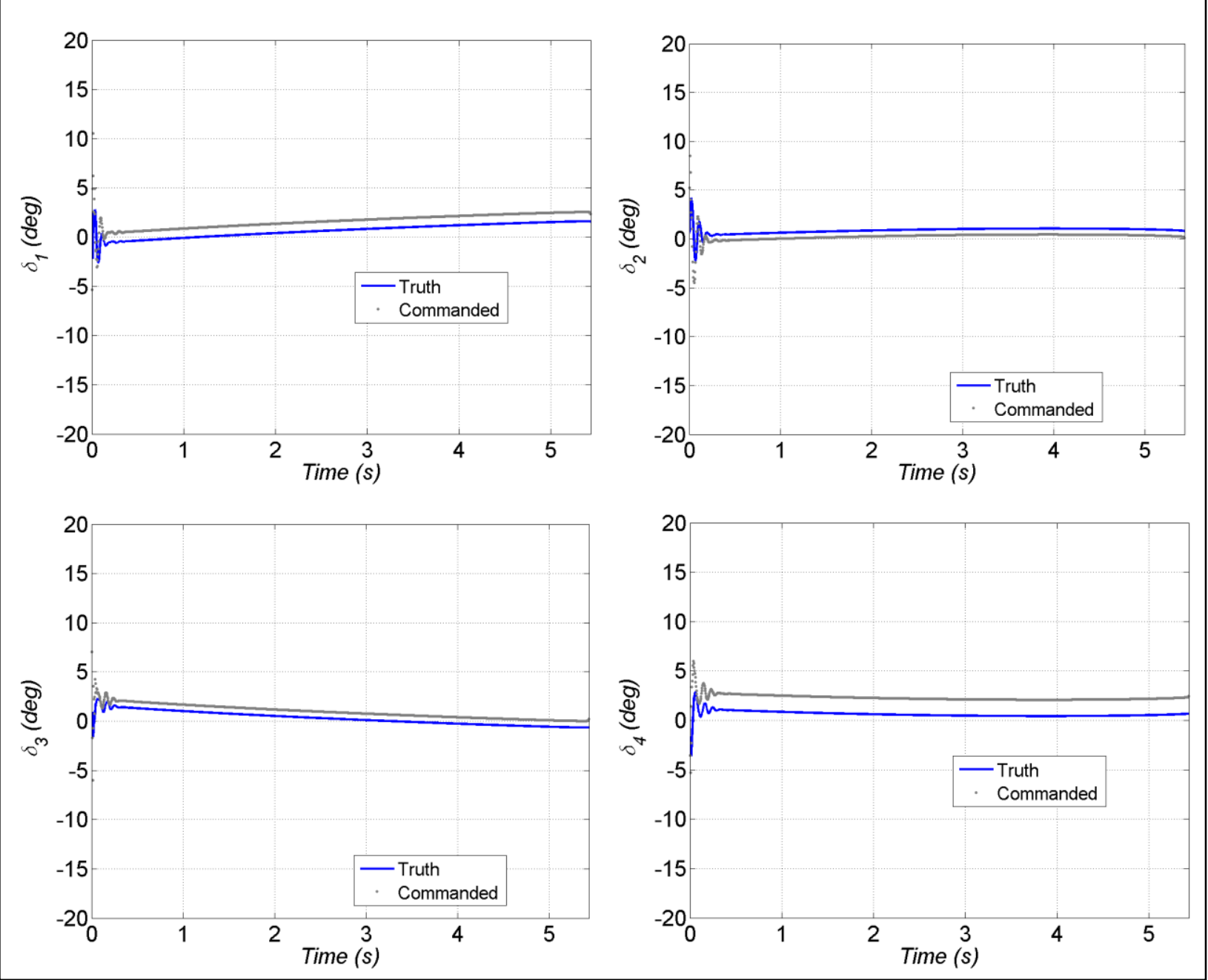

Fig. 35 Canard deflection histories for representative flight of Configuration 2

Canard deflection saturation (i.e., reaching a maximum value, for example, of $10^{\circ}$ in Fig. 35) in turn saturates the lateral acceleration (i.e., constant $\dot{v}$ or $\dot{w}$ in Fig. 34, for example, of $50 \mathrm{~m} / \mathrm{s}^{2}$ ) at the limit based on the airframe characteristics. The lack of saturation in the results for the canard commands and controlled responses in Figs. 34 and 35 confirms that the design methodology yielded an airframe capable of successful intercept for this class of launch and target conditions. 


\section{Conclusions}

The primary purpose of this investigation was to design a stable platform for the LCHAW moving target mission program. This study demonstrated the need to include canard interaction effects when determining the stability a of body-fin-canard projectile configuration. The bodyfin-canard projectile that had been designed using the aerodynamic data obtained for the bodyfin portion of the projectile without canards and then adding the canard data from a separate source was found to be statically unstable across the Mach number regime of interest when the aerodynamic data for the components were obtained from a single CFD simulation. The component aerodynamic data from the single CFD simulation (i.e., canard interaction effects included) was used in a parameter sensitivity study to determine a new configuration that would meet stability requirements. The results of the parameter sensitivity study plus geometric constraints were used to design a second configuration for consideration as the demonstration platform for the LCHAW moving target mission program.

Using form factors on data that already included the canard interaction effects produced reasonable results - the updated configuration remained statically stable with a trim angle of approximately $4^{\circ}$ with deflected canards. Comparing the computational aerodynamic coefficients to the predicted values proved that incorporating interaction effects into the component aerodynamic coefficient, even if scaling was necessary, was a reasonable assumption during the design process. A complete aerodynamic model was developed for this second configuration, including roll damping coefficient, at a single Mach number using CFD simulations. The aerodynamic model was used as input for both linear and non-linear flight mechanics and control simulations. These simulations showed that this configuration would meet maneuverability and range requirements as well as stability requirements.

During the course of the design process, a number of interesting phenomena were discovered that need to be investigated further. A most basic concern was the inability of the ballistic fits to predict $C_{X_{o}}$ and $C_{l_{o}}$ accurately. Additional work on the fits should clear up this issue. Currently, only configurations with non-deflected canards were considered. However, there are 2 items that lend themselves to a more in depth investigation once canard deflections are considered. First, the effect of canard deflection on canard stall angle and projectile trim angles will need to be investigated. Second, the interaction effects between the canard and both the body and the fins when the canards are deflected will need further research. It is likely that tracing the canard streamlines emanating from both the inboard and tip locations will be necessary to determine the full extent of the interactions. The interaction effects will likely be critical in understanding and predicting the projectile maneuver behavior, especially at higher angles of attack. 


\section{References}

1. Fresconi F; Brown G, Celmins I, DeSpirito J, Ilg M, Maley J, Magnotti P. Scanlan A, Stout C, Vazquez E. Very affordable precision projectile technology development and flight demonstrations. Aberdeen Proving Ground (MD): Army Research Laboratory (US); 2011. Report No.: ARL-TR-5460.

2. Fresconi F, Harkins T. Aerodynamic characterizations of asymmetric and maneuvering 105-, 120-, and 155-mm fin-stabilized projectiles derived from telemetry experiment. Aberdeen Proving Ground (MD): Army Research Laboratory (US); 2011. Report No.: ARL-TR-5495. Also available at http://www.arl.army.mil/www/default.cfm?technical_report=6185.

3. Fresconi F, Celmins I, Ilg M, Maley J. Projectile roll dynamics and control with a low-cost skid-to-turn maneuver system. Aberdeen Proving Ground (MD): Army Research Laboratory (US); 2013. Report No.: ARL-TR-6363. Also available at http://www.arl.army.mil/www/default.cfm?technical_report=6696.

4. Silton SI, Fresconi FE. High maneuverability airframe: initial investigation of configuration aft end for increased stability, range, and maneuverability. Aberdeen Proving Ground (MD): Army Research Laboratory (US); 2013. Report No.: ARL-TR-6585. Also available at http://www.arl.army.mil/www/default.cfm?technical_report=6907.

5. DeSpirito J, Vaughn ME, Washington WD. Numerical investigation of aerodynamics of canard-controlled missile using planar and grid tail fins, part i: supersonic flow. Aberdeen Proving Ground (MD): Army Research Laboratory (US); 2002. Report No.: ARL-TR-2848. Also available at http://www.arl.army.mil/www/default.cfm?technical_report=651.

6. DeSpirito J, Vaughn ME, Washington WD. Numerical investigation of aerodynamics of canard-controlled missile using planar and grid tail fins, part ii: subsonic and transonic flow. Aberdeen Proving Ground (MD): Army Research Laboratory (US); 2004. Report No.: ARLTR-3162. Also available at http://www.arl.army.mil/www/default.cfm?technical_report=819.

7. Beresh SJ, Smith JA, Henfling JF, Grasser TW, Spillers RW. Interaction of a fin trailing vortex with a downstream control surface. J of Spacecraft and Rockets. 2009;46(2):318-328.

8. Dassault Systems SolidWorks Corporation. SolidWorks. Waltham (MA); 2012.

9. Metacomp Technologies, Inc. CFD ${ }^{++}$user manual. Agoura Hills (CA); 2011.

10. Pulliam TH, Steger JL. On implicit finite-difference simulations of three-dimensional flow. AIAA Journal. 1982;18(2):159-167. 
11. Peroomian O, Chakravarthy S, Goldberg U. A 'grid-transparent' methodology for CFD. AIAA Paper 1997-0724. Proceeding of 35 ${ }^{\text {th }}$ Aerospace Sciences Meeting and Exibit. 1997 Jan 6-10; Reno, NV.

12. Peroomian O, Chakravarthy S, Palaniswamy S, Goldberg U. Convergence acceleration for unified-grid formulation using preconditioned implicit relaxation. AIAA Paper 1998-0116. Proceedings of the $36^{\text {th }}$ Aerospace Sciences Meeting and Exhibit. 1998 Jan 12-14; Reno, NV.

13. Goldberg UC, Peroomian O, Chakravarthy S. A wall-distance-free k- $\varepsilon$ model with enhanced near-wall treatment. ASME Journal of Fluids Engineering. 1998;120: 457-462.

14. Metacomp Technologies, Inc. MIME User Manual; Agoura Hills (CA); 2010.

15. DeSpirito J, Silton S, Weinacht P. Navier-stokes predictions of dynamic stability derivatives: evaluation of steady-state methods. Journal of Spacecraft and Rockets. 2009;46(6):1142-1154.

16. Fresconi FE, Celmins I, Silton SI. Theory, guidance, and flight control for high maneuverability projectiles. Aberdeen Proving Ground (MD): Army Research Laboratory (US); 2014. Report No.: ARL-TR-6767. Also available at http://www.arl.army.mil/www/default.cfm?technical_report=6972 
INTENTIONALLY LEFT BLANK. 
Appendix A. Transformations 
Transformations between coordinate systems were an integral part of the analysis presented in the main body of this report. The transformations ensured that the aerodynamic coefficient database created from the computational fluid dynamics (CFD) analysis was properly utilized in both the optimization routine as well as in the flight mechanics and control analysis.

The first transformation was completed to move from the CFD coordinate system to the projectile body coordinate system. The CFD coordinate system is the right-hand coordinate system with the $\mathrm{x}$-axis aligned with the centerline of the projectile positive from nose to tail and the z-axis positive up, as defined in Fig. A-1. The body-fixed coordinate system is defined in Fig. A-2. A major difference in these 2 coordinate systems stems from the definition of the velocity vector; in the CFD coordinate system the air moves over the projectile, while the projectile moves through the air in the body-fixed coordinate system. Thus, $\vec{V}_{C G / I}=-\vec{V}_{\text {wind }}$.

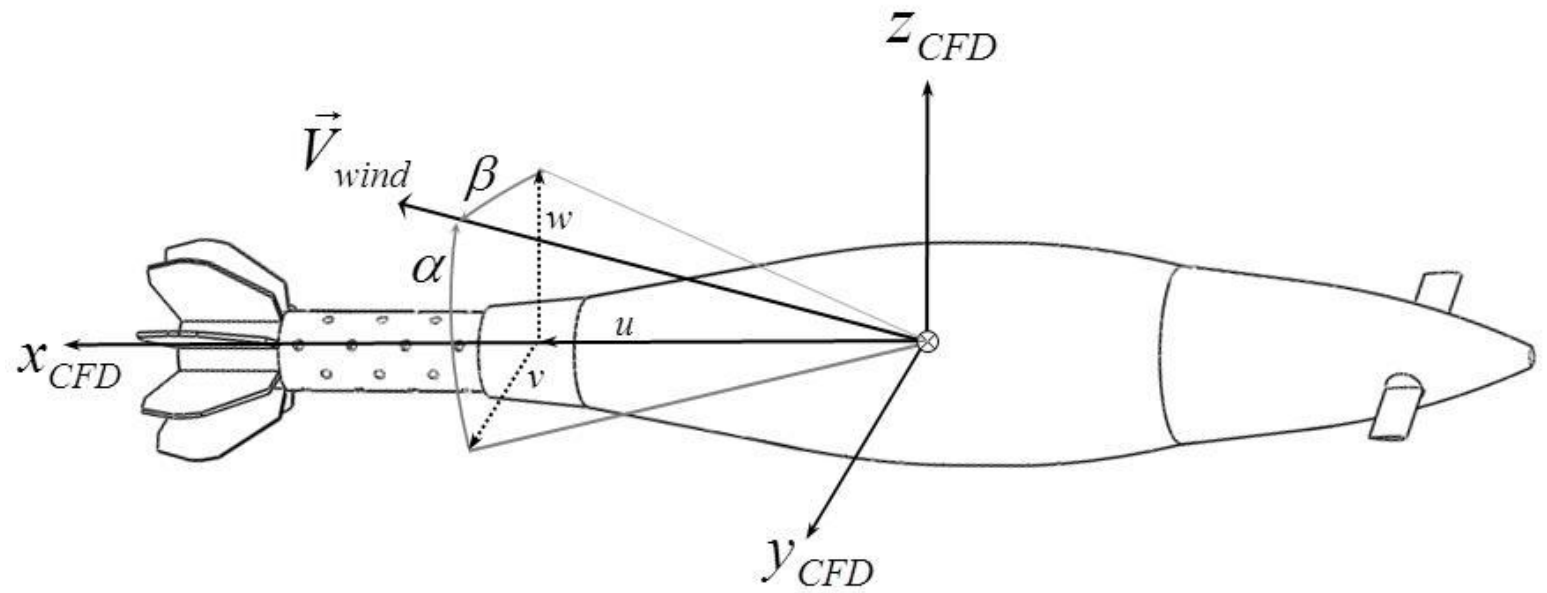

Fig. A-1 CFD coordinate system and aerodynamic angles 


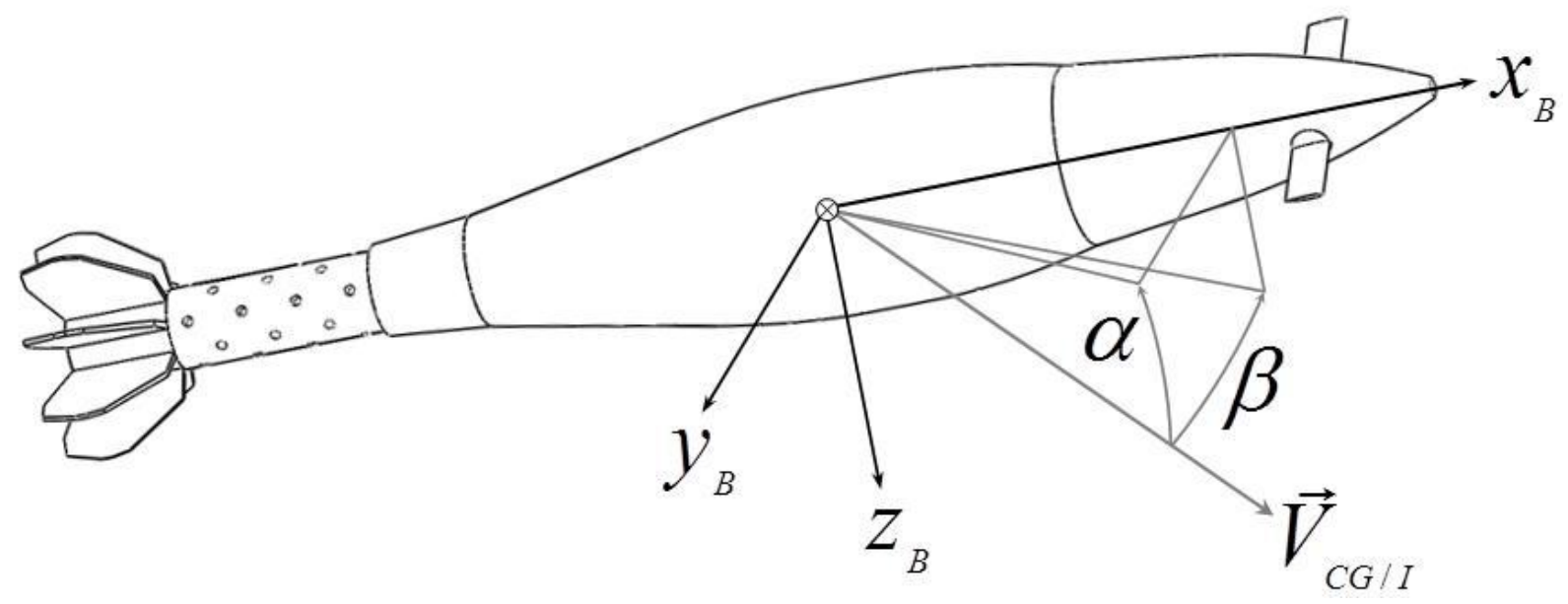

Fig. A-2 Body-fixed coordinate system and aerodynamic angles

The transformation matrix to convert from CFD to projectile body coordinates, $\vec{T}_{C B}$, is given by Eq. A-1. Equation A-2 is then used to convert between the 2 coordinate systems

$$
\begin{gathered}
\vec{T}_{C B}=\left[\begin{array}{ccc}
-1 & 0 & 0 \\
0 & 1 & 0 \\
0 & 0 & -1
\end{array}\right] \\
{\left[\begin{array}{l}
X \\
Y \\
Z
\end{array}\right]_{B}=\vec{T}_{C B}\left[\begin{array}{l}
X \\
Y \\
Z
\end{array}\right]_{C F D}}
\end{gathered}
$$

where $\left[\begin{array}{l}X \\ Y \\ Z\end{array}\right]$ are the 3 components of the force or moment coefficients or velocity. Equation A-2 only transforms the velocity vector from CFD coordinates to body coordinates; the velocity vector remains the wind velocity vector. To complete the transformation to body coordinates as defined in Fig. A-2, $\vec{V}_{\text {wind }}$ must be converted to $\vec{V}_{C G / I}$ using Eq. A-3.

$$
\vec{V}_{C G / I}=\left[\begin{array}{ccc}
-1 & 0 & 0 \\
0 & -1 & 0 \\
0 & 0 & -1
\end{array}\right] \vec{V}_{\text {wind }}
$$

It was determined that the canard components should be analyzed in the local blade coordinate system rather than in the projectile body coordinate system. As such, the force and moments coefficients for each of the canards must further be transformed from the projectile body coordinate to the local blade coordinate system. The local blade coordinate system is defined as the $\mathrm{x}$-axis parallel to $x_{B}$. The $\mathrm{y}$-axis runs along the span of the canard from the root to the tip. The z-axis runs perpendicular to follow the right hand rule. The transformation matrix to convert 
from the projectile body coordinate system to the local blade coordinate system of the $i^{\text {th }}$ canard, $\vec{T}_{B M_{i}}$, is given by Eq. A-4, where $\phi_{M_{i}}$ is the roll orientation of the given canard. The roll

orientation of the canards is determined by looking from the rear of the projectile (Fig. A-3). The roll orientation of Canard 4 (i.e. $M_{4}$ ) is $\phi_{M_{i}}=45^{\circ}$ for this study. In Fig. A-3, the sign convention for canard deflection is for the trailing edge. The aerodynamic coefficients in the local blade coordinate system of the $i^{\text {th }}$ canard are then determined by Eq. A-5.

$$
\begin{aligned}
\vec{T}_{B M_{i}}= & {\left[\begin{array}{ccc}
1 & 0 & 0 \\
0 & \cos \left(\phi_{M_{i}}\right) & \sin \left(\phi_{M_{i}}\right) \\
0 & -\sin \left(\phi_{M_{i}}\right) & \cos \left(\phi_{M_{i}}\right)
\end{array}\right] } \\
& {\left[\begin{array}{l}
X \\
Y \\
Z
\end{array}\right]_{M_{i}}=\vec{T}_{B M_{i}}\left[\begin{array}{l}
X \\
Y \\
Z
\end{array}\right]_{B} }
\end{aligned}
$$

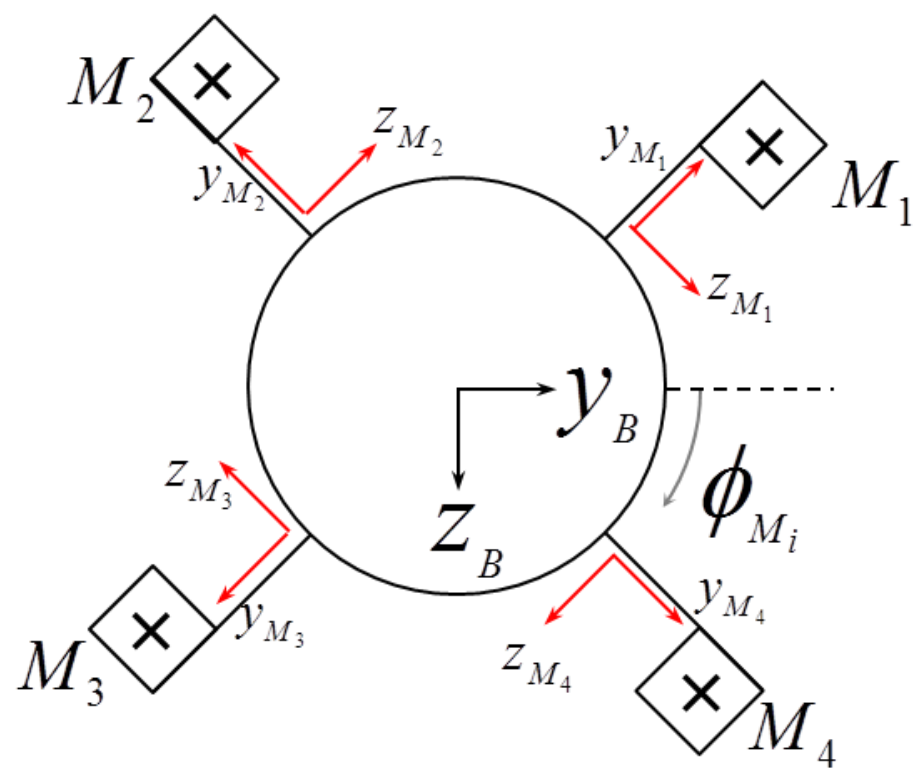

Fig. A-3 Definition of canard orientation when looking from the rear of the projectile relative to body coordinates

The final transformations completed in this paper are to change the aerodynamic coefficients in the projectile body frame to ballistic coefficients (Fig. A-4). To obtain the ballistic coefficients, only the sign on vertical force (i.e., normal force) and axial force must be inverted as $C_{N}$ is defined positive up and $C_{A}$ positive aft for positive $\alpha$, such that

$$
\begin{aligned}
& C_{N}=-C_{Z_{B}} \\
& C_{A}=-C_{X_{B}}
\end{aligned}
$$


Within this paper, $C_{A}$ and $C_{X}$ are used interchangeably to indicate axial force coefficient as Eq. A-7 was applied early in the transformation process without consequence.

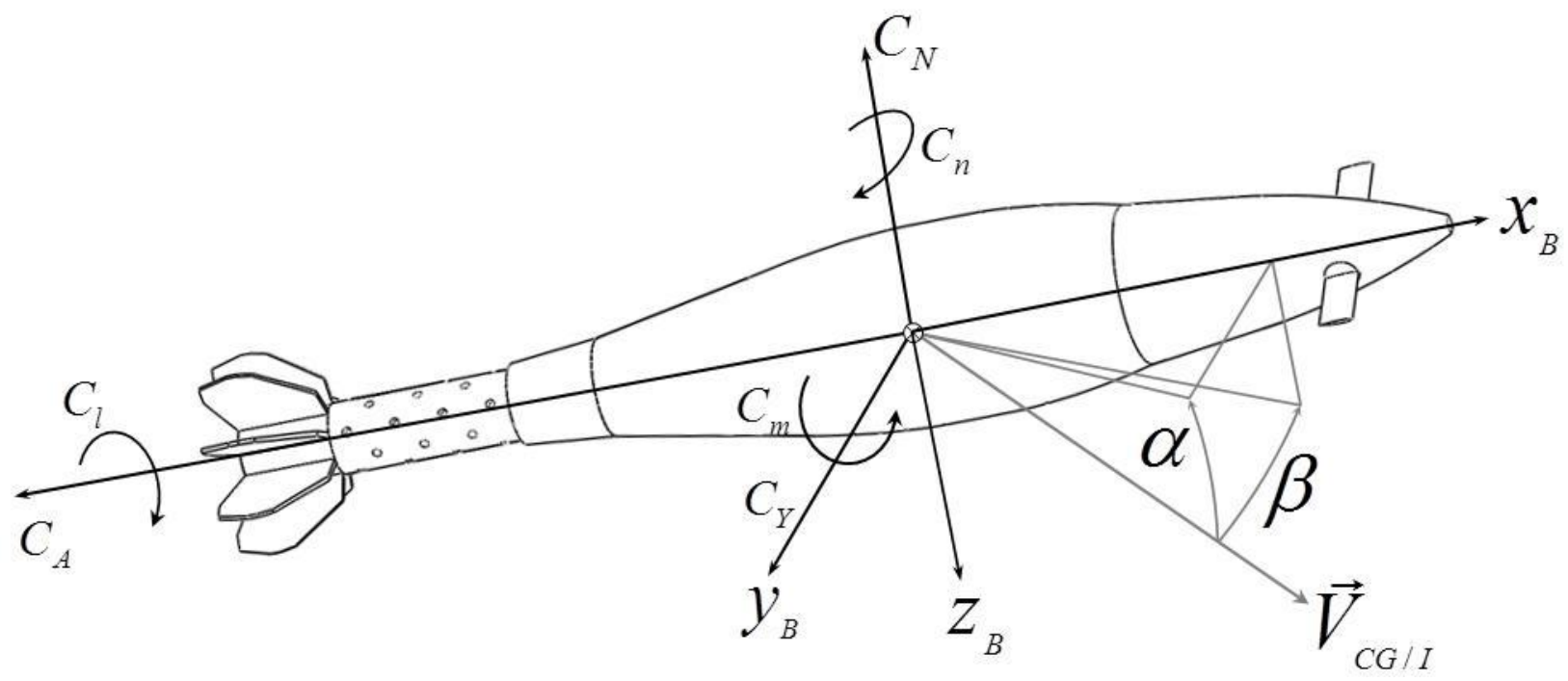

Fig. A-4 Definition of ballistic coefficients and aerodynamic angles in the body coordinate system 
INTENTIONALLY LEFT BLANK. 
Appendix B. Coefficient Fits for Configurations 1a and 1b 
Best fits were determined for each of the components for both Configurations $1 \mathrm{a}$ and $1 \mathrm{~b}$ at all 3 Mach numbers. The resulting fits were checked for accuracy against the CFD for both the individual component and for the sum of the components. The value of the total fit was obtained by summing the component fits; the total CFD value was obtained directly from the CFD (i.e., the CFD components were not summed). Using the total CFD value allowed for an additional check that all component values were being correctly utilized.

Figures B-1 and B-2 show the resulting fits for Configuration 1a at Mach 0.5 for the body and fin components and each of the canard components, respectively. Figure B-3 shows the results of the component buildup.
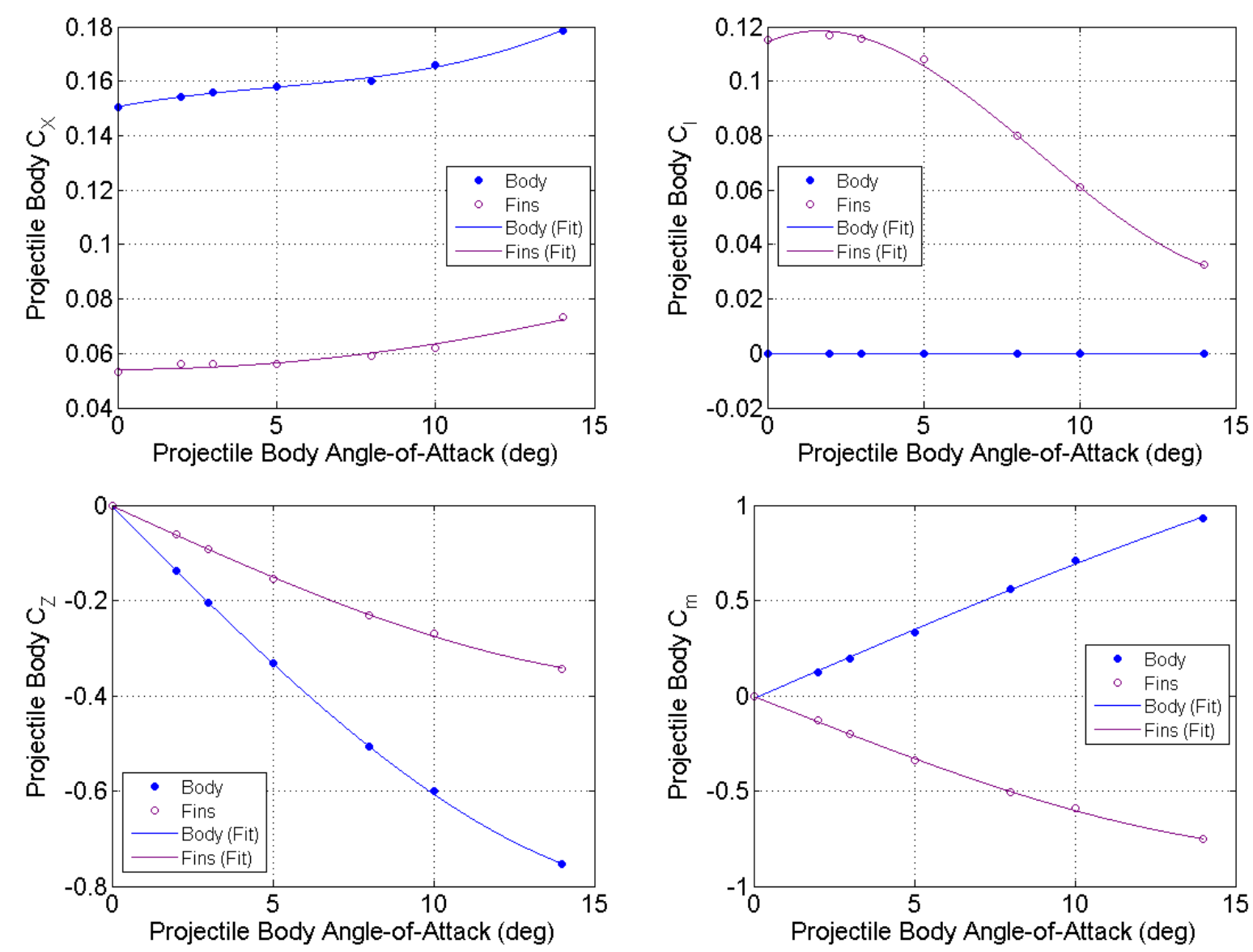

Fig. B-1 Body and fin component fits for Configuration 1a, Mach 0.5 

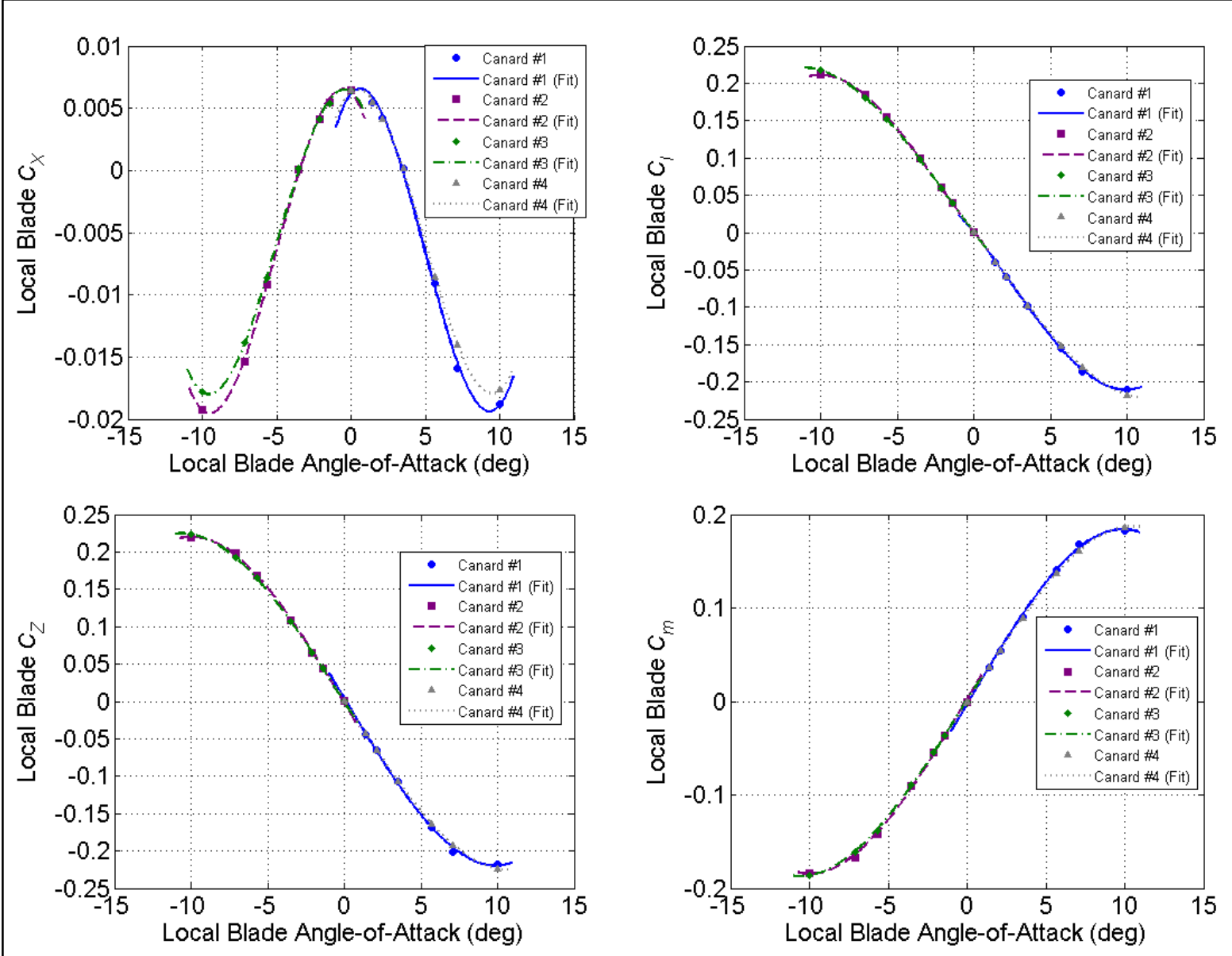

Fig. B-2 Individual canard fits in local canard coordinate system for Configuration 1a, Mach 0.5 

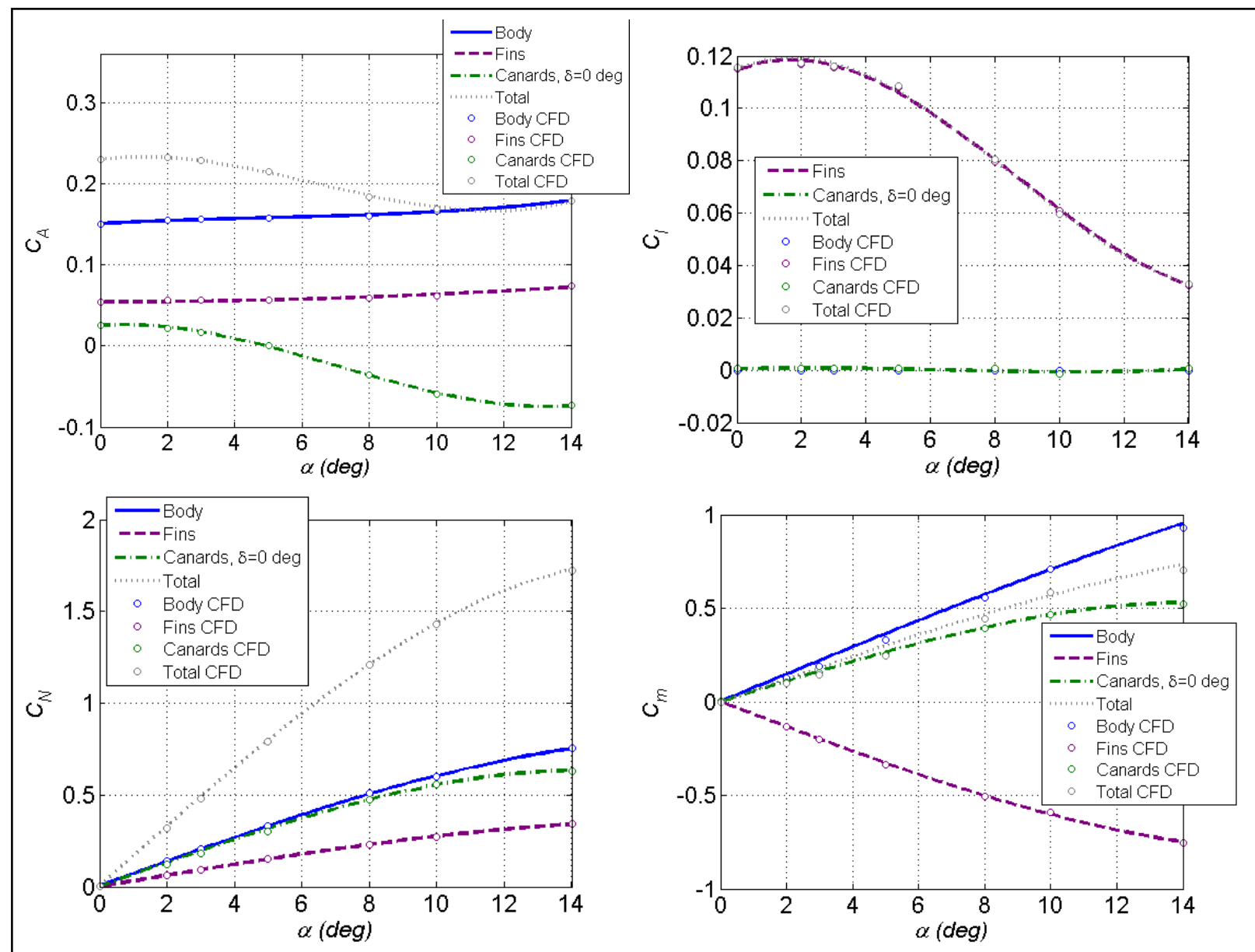

Fig. B-3 Component fit build up comparison to total CFD coefficients for Configuration 1a, Mach 0.5

Figures B-4 and B-5 show the resulting fits for Configuration 1a at Mach 0.65 for the body and fin components and each of the canard components, respectively. Figure B-6 shows the results of the component buildup. 

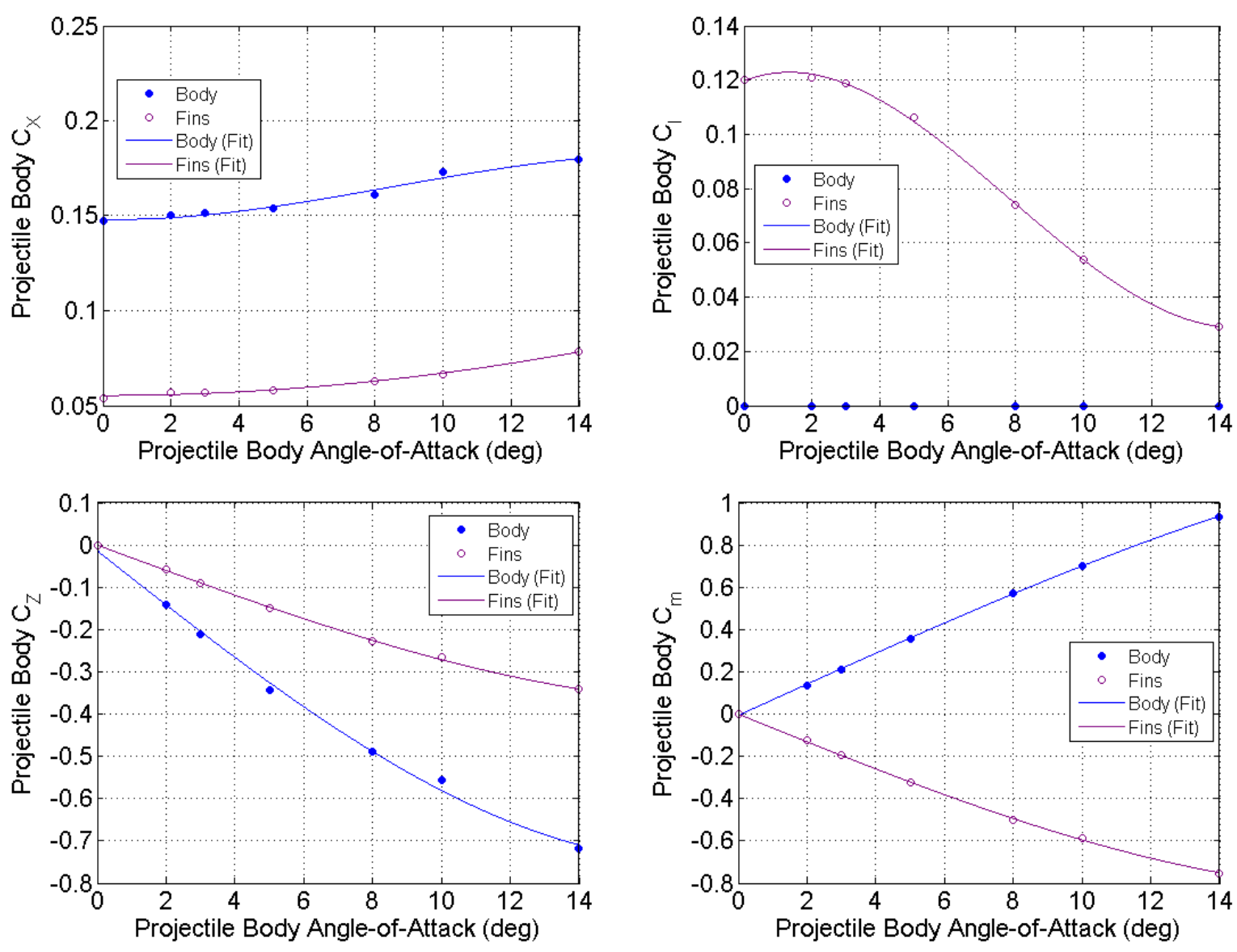

Fig. B-4 Body and fin component fits for Configuration 1a, Mach 0.65 

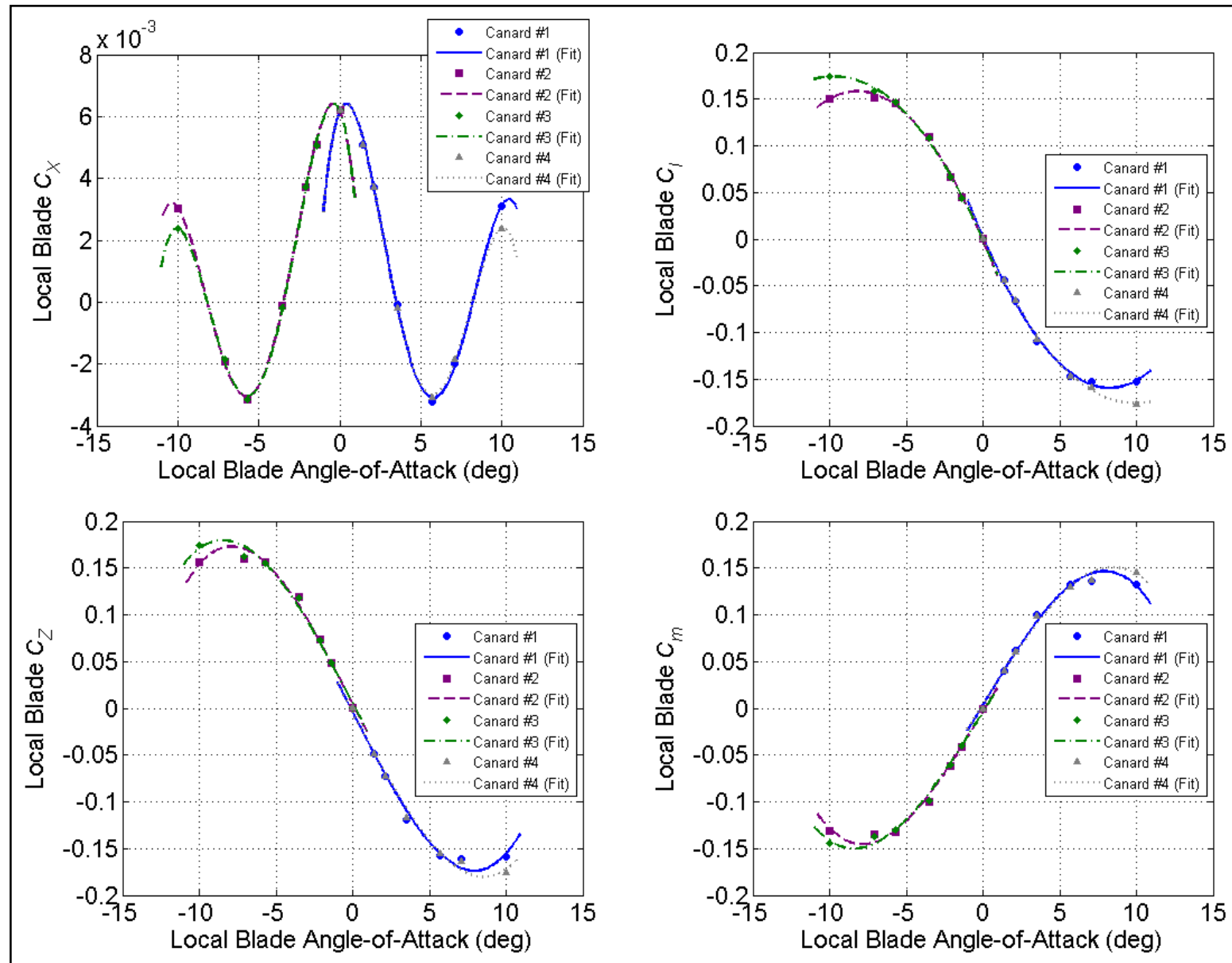

Fig. B-5 Individual canard fits in local canard coordinate system for Configuration 1a, Mach 0.65 

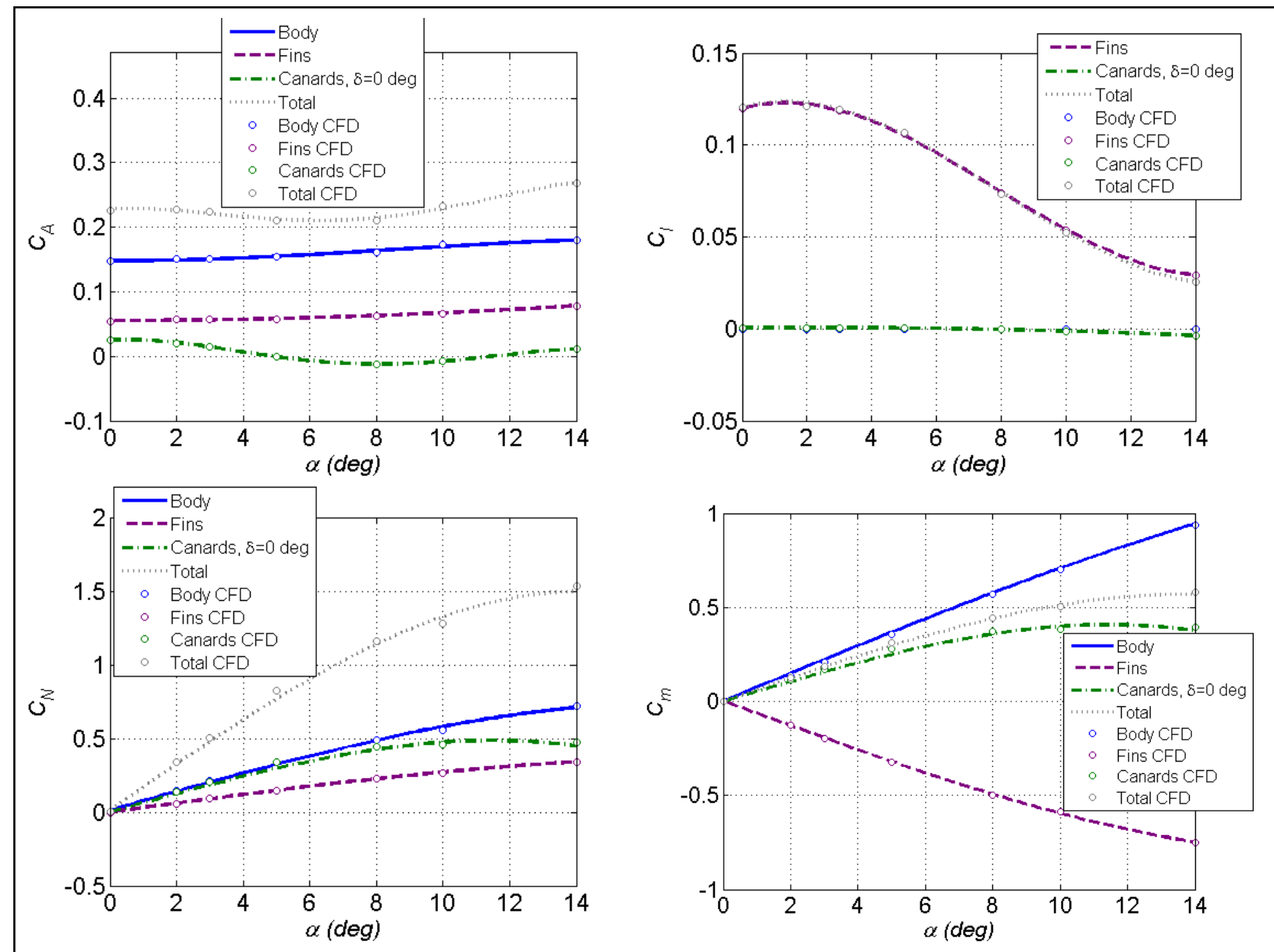

Fig. B-6 Component fit build up comparison to total CFD coefficients for Configuration 1a, Mach 0.65

Figures B-7 and B-8 show the resulting fits for Configuration 1a at Mach 0.8 for the body and fin components and each of the canard components, respectively. Figure B-9 shows the results of the component buildup. 

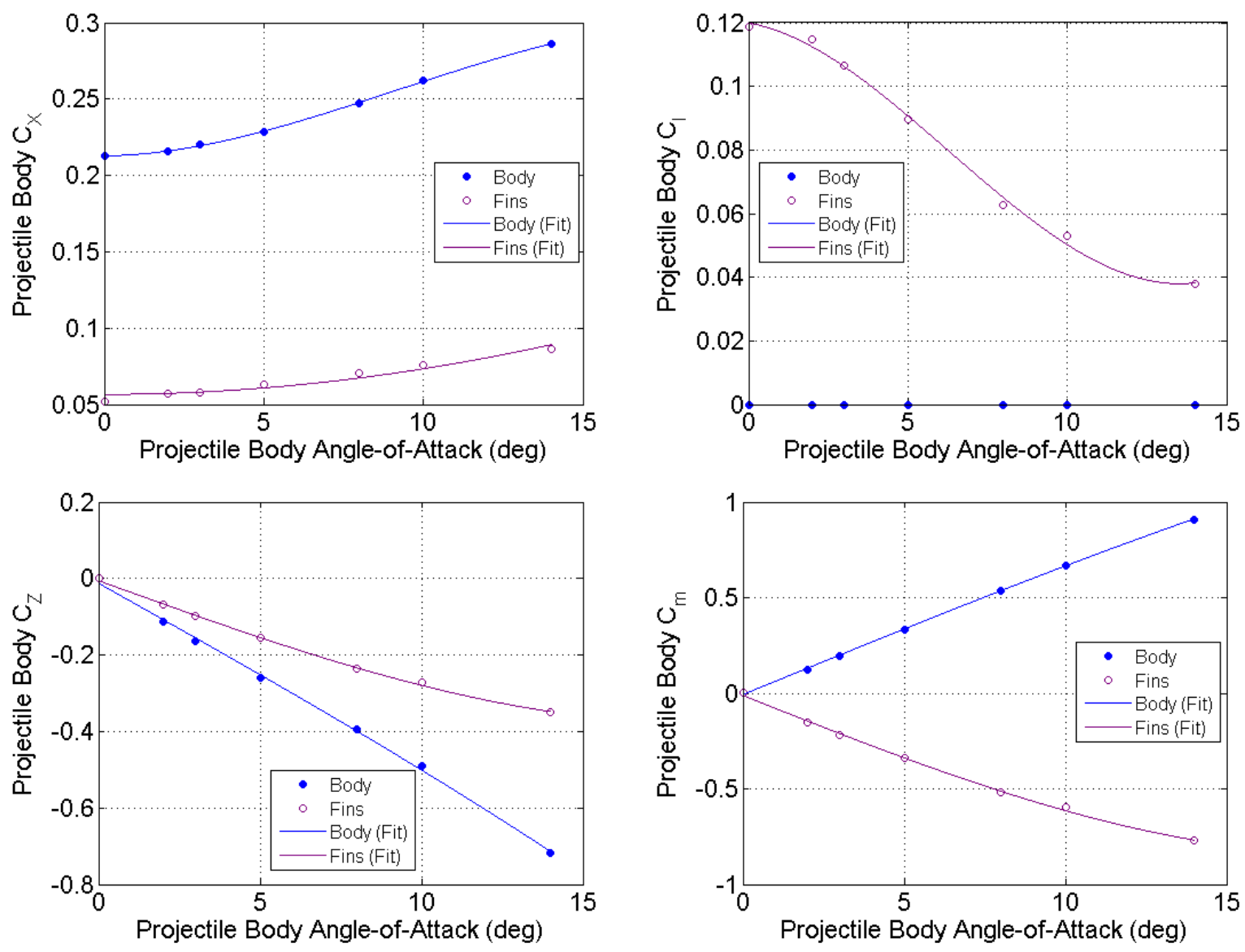

Fig. B-7 Body and fin component fits for Configuration 1a, Mach 0.8 

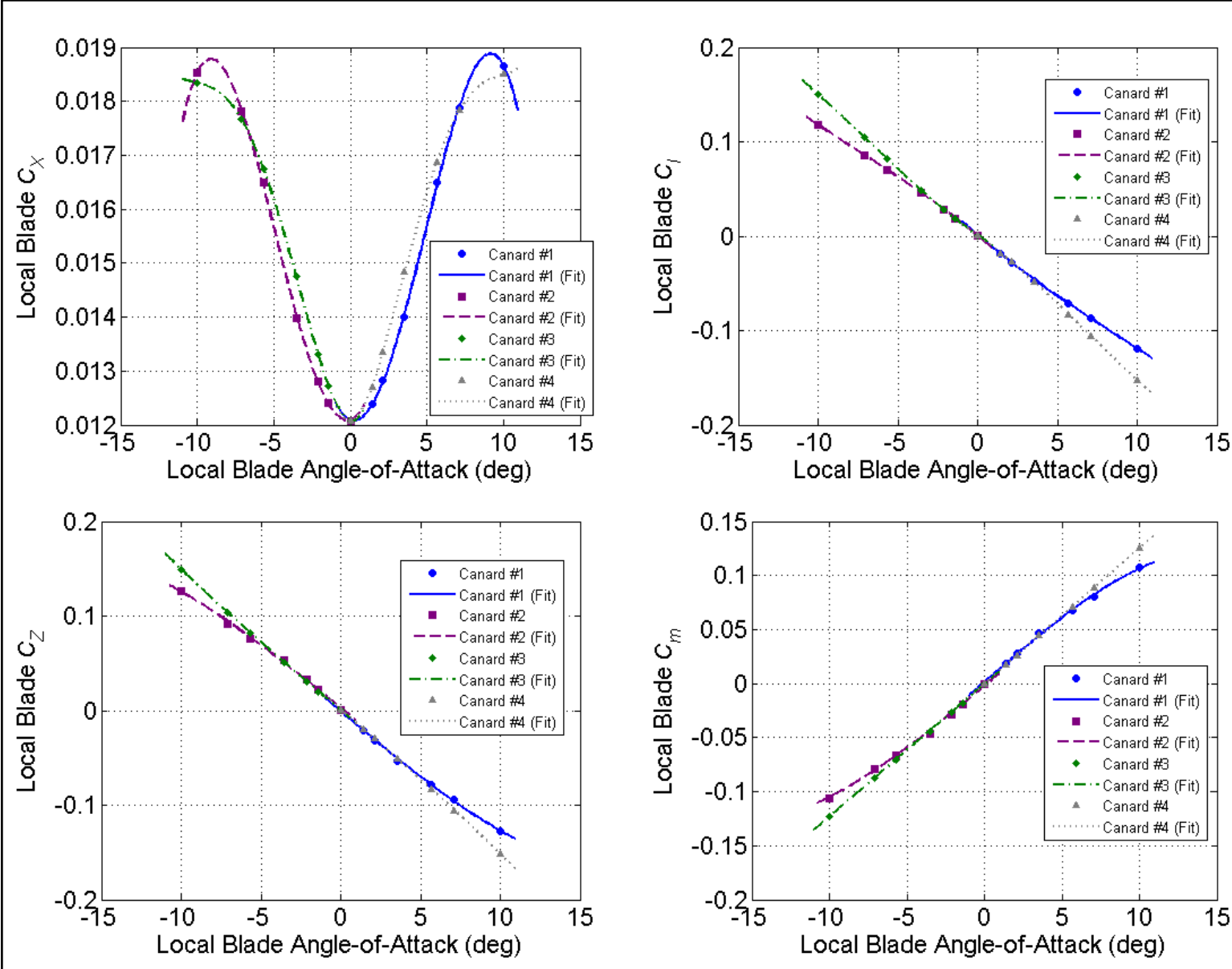

Fig. B-8 Individual canard fits in local canard coordinate system for Configuration 1a, Mach 0.8 

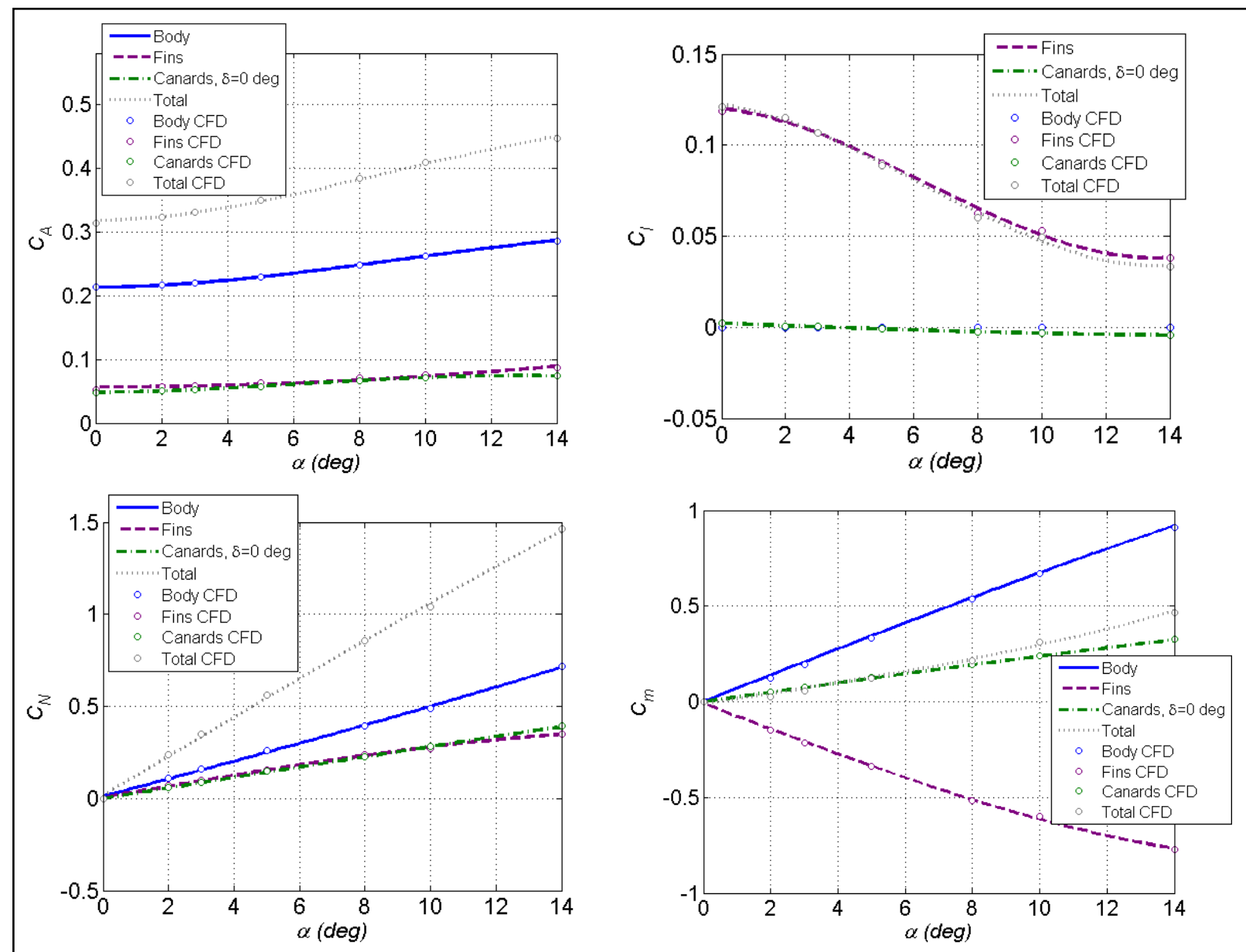

Fig. B-9 Component fit build up comparison to total CFD coefficients for Configuration 1a, Mach 0.8

Figures B-10 and B-11 show the resulting fits for Configuration $1 \mathrm{~b}$ at Mach 0.5 for the body and fin components and each of the canard components, respectively. Figure B-12 shows the results of the component buildup. 

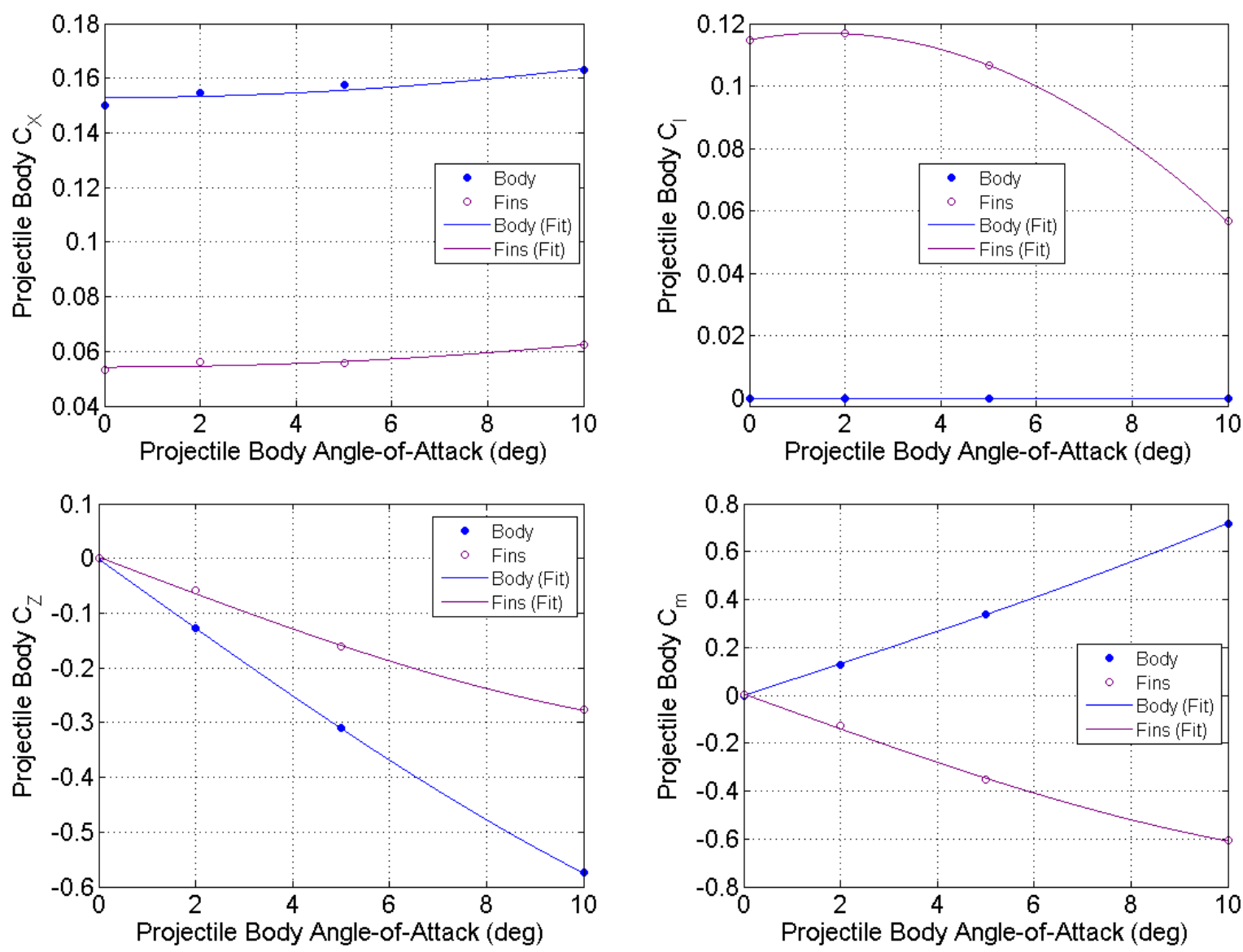

Fig. B-10 Body and fin component fits for Configuration 1b, Mach 0.5 

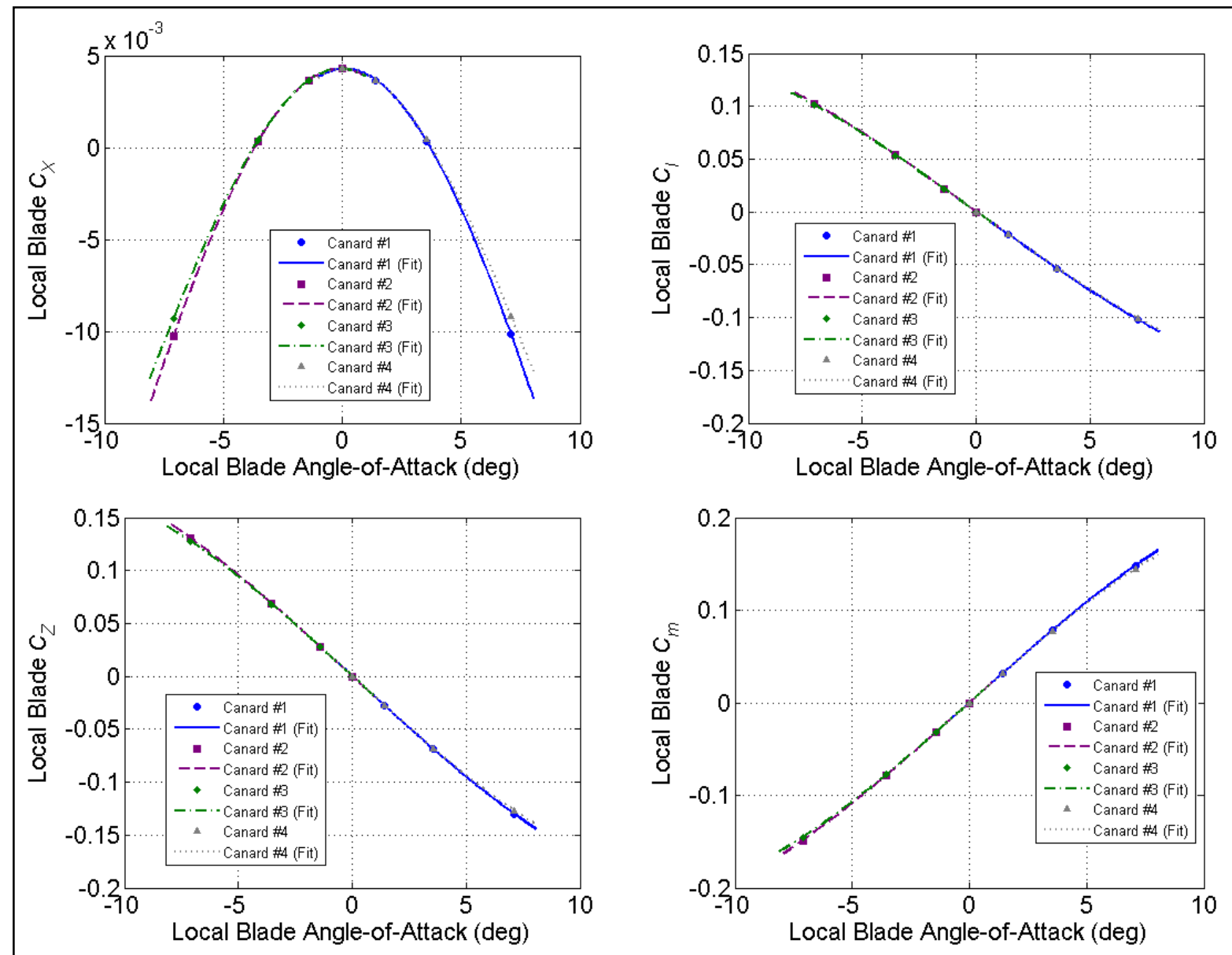

Fig. B-11 Individual canard fits in local canard coordinate system for Configuration 1b, Mach 0.5 

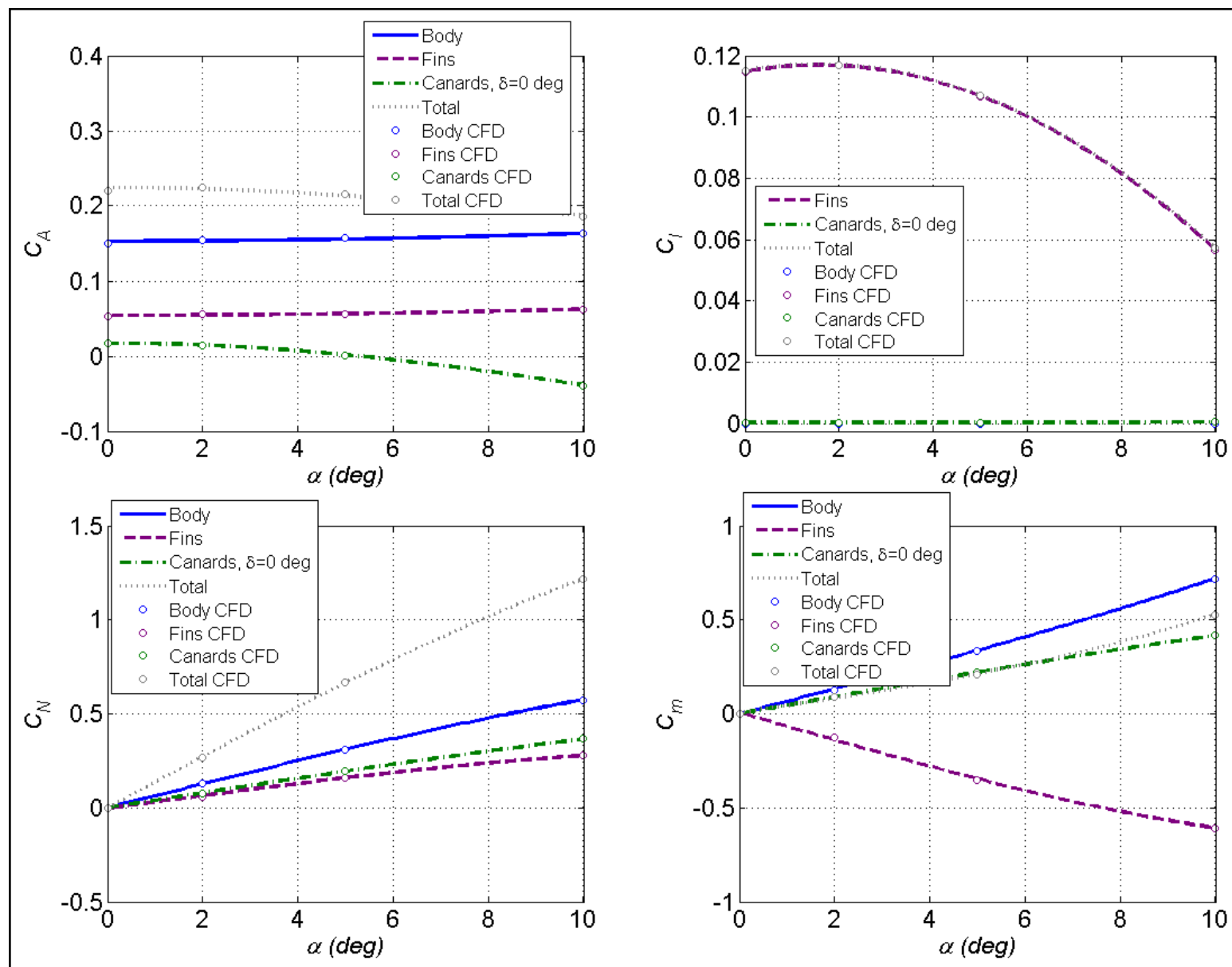

Fig. B-12 Component fit build up comparison to total CFD coefficients for Configuration 1b, Mach 0.5

Figures B-13 and B-14 show the resulting fits for Configuration $1 \mathrm{~b}$ at Mach 0.65 for the body and fin components and each of the canard components, respectively. Figure B-15 shows the results of the component buildup. 

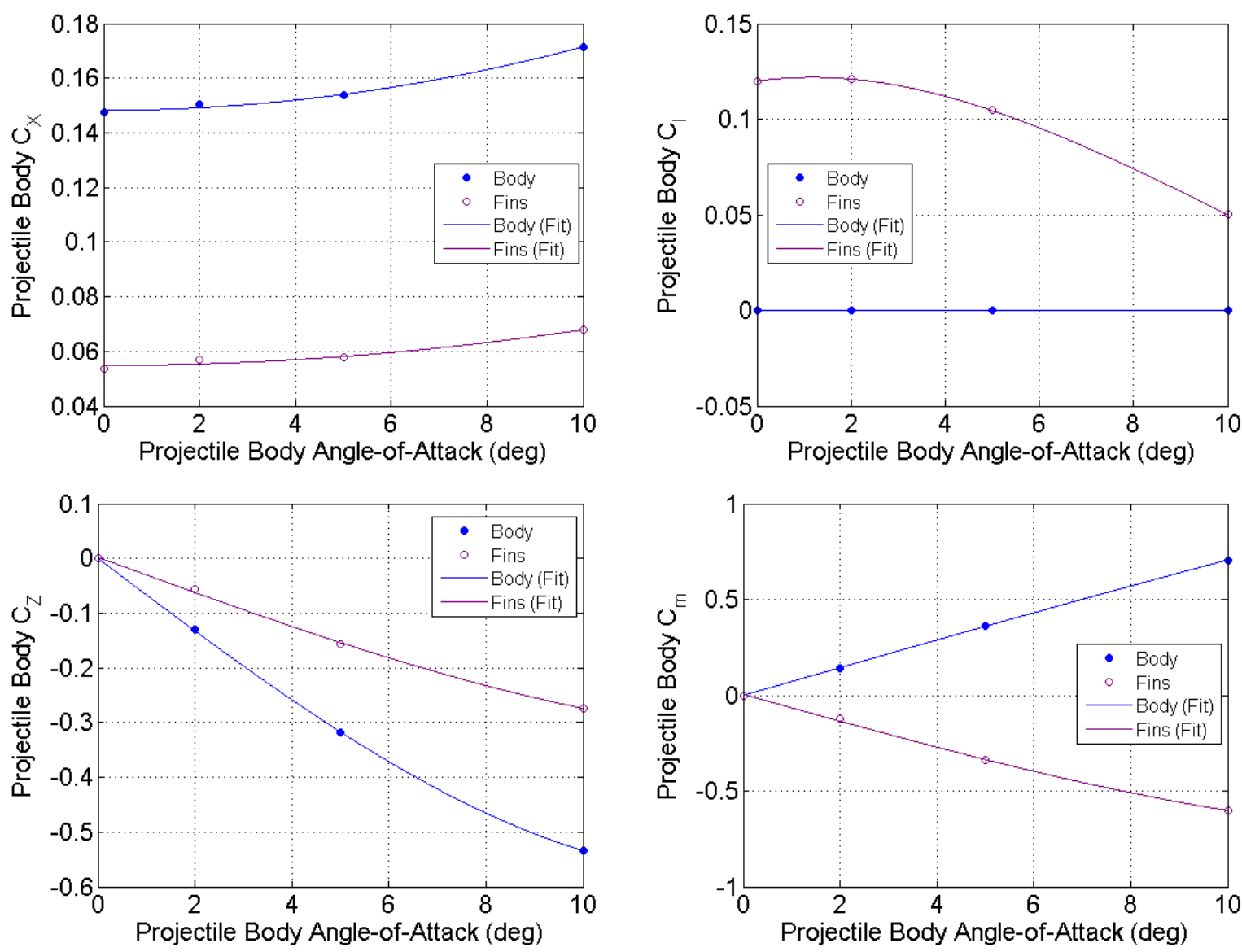

Fig. B-13 Body and fin component fits for Configuration 1b, Mach 0.65 

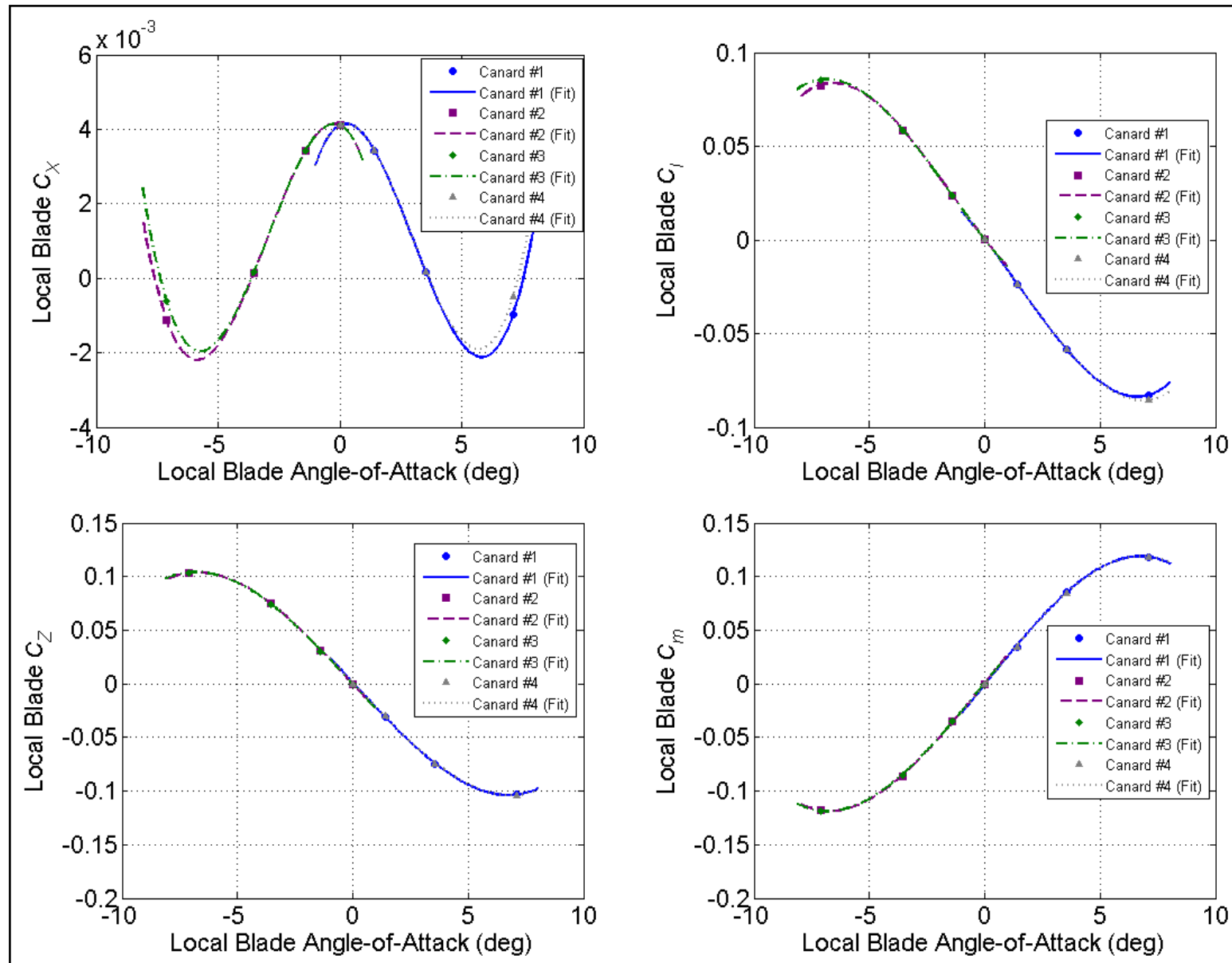

Fig. B-14 Individual canard fits in local canard coordinate system for Configuration 1b, Mach 0.65 

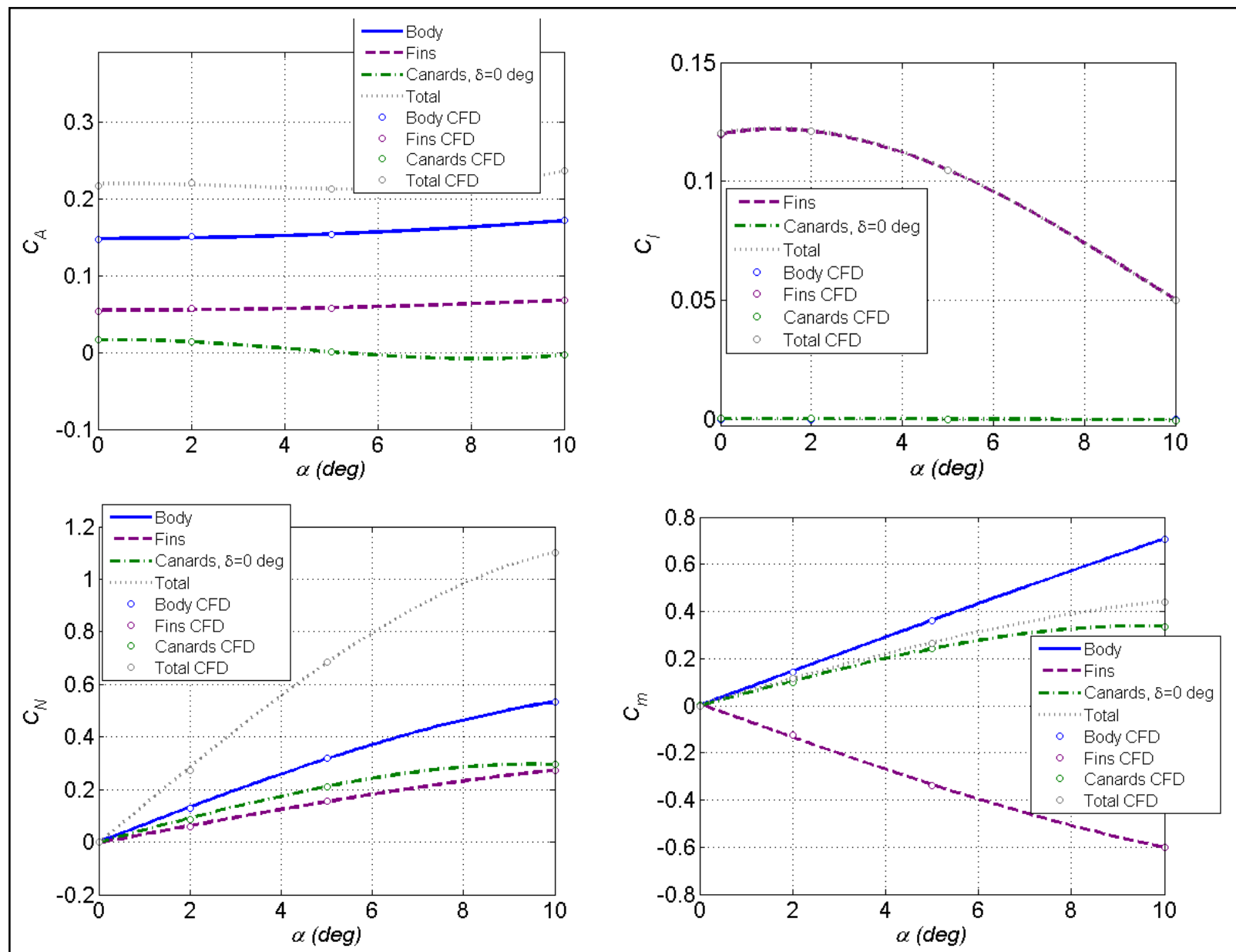

Fig. B-15 Component fit build up comparison to total CFD coefficients for Configuration 1b, Mach 0.65

Figures B-16 and B-17 show the resulting fits for Configuration $1 \mathrm{~b}$ at Mach 0.8 for the body and fin components and each of the canard components, respectively. Figure B-18 shows the results of the component buildup. 

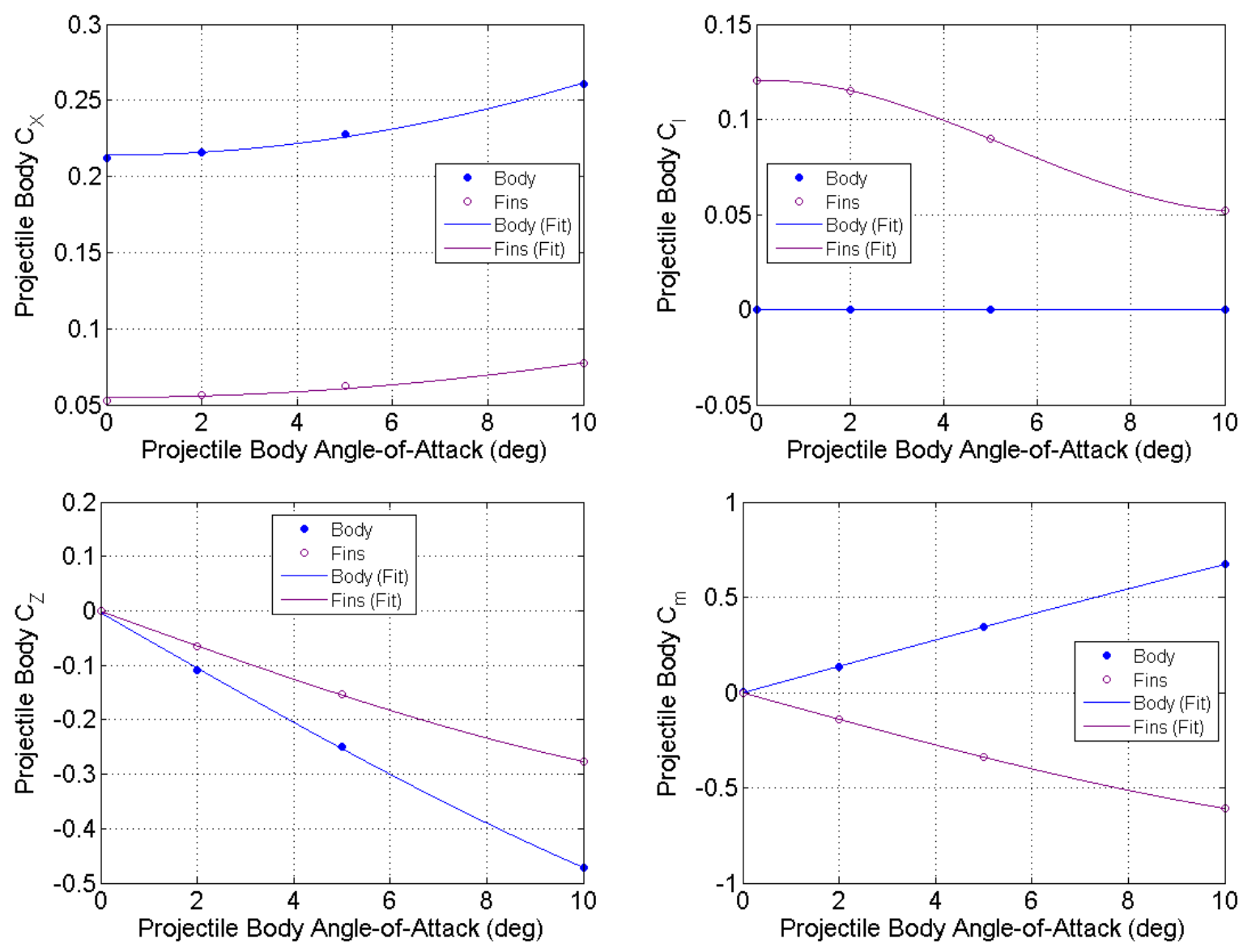

Fig. B-16 Body and fin component fits for Configuration 1b, Mach 0.8 

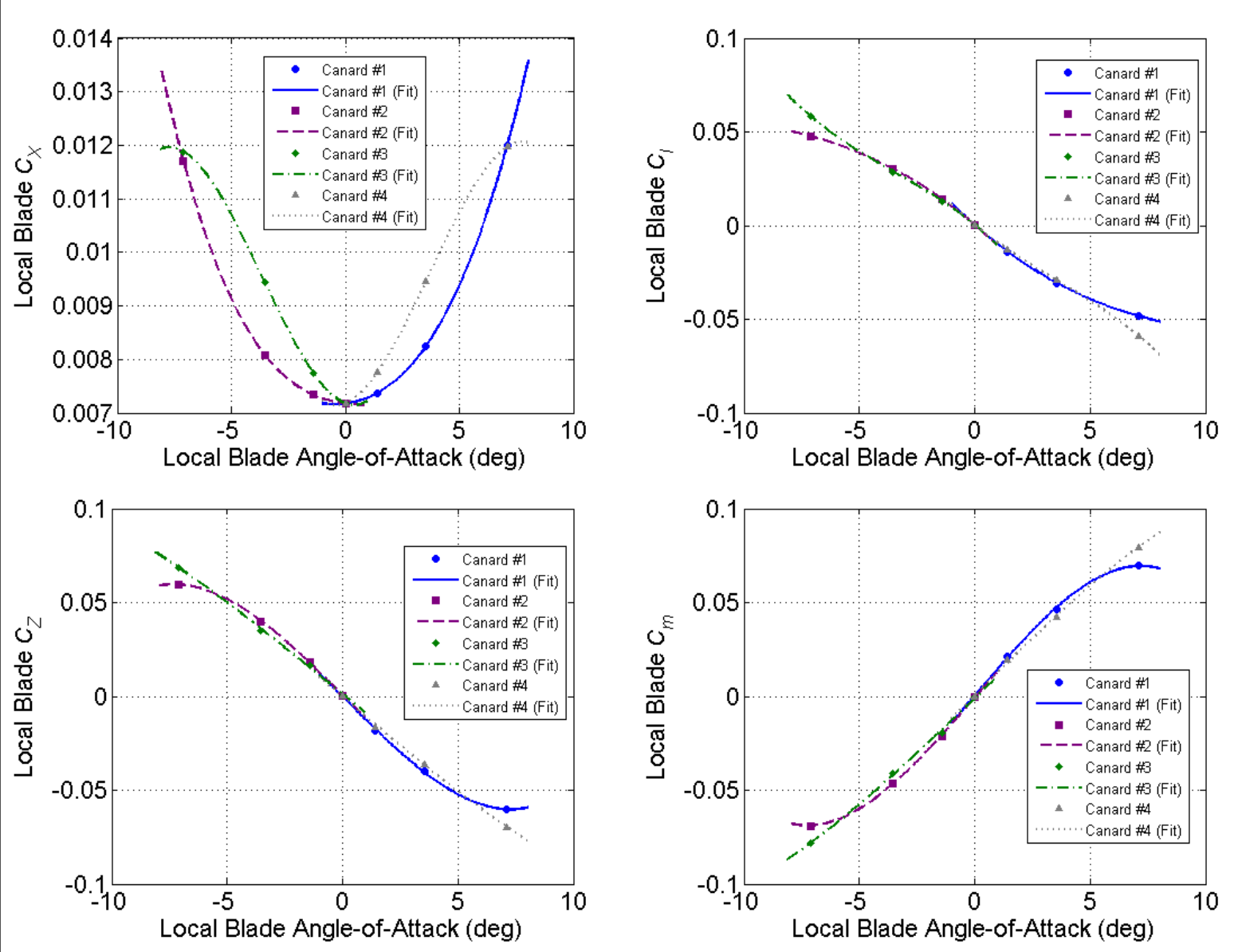

Fig. B-17 Individual canard fits in local canard coordinate system for Configuration 1b, Mach 0.8 

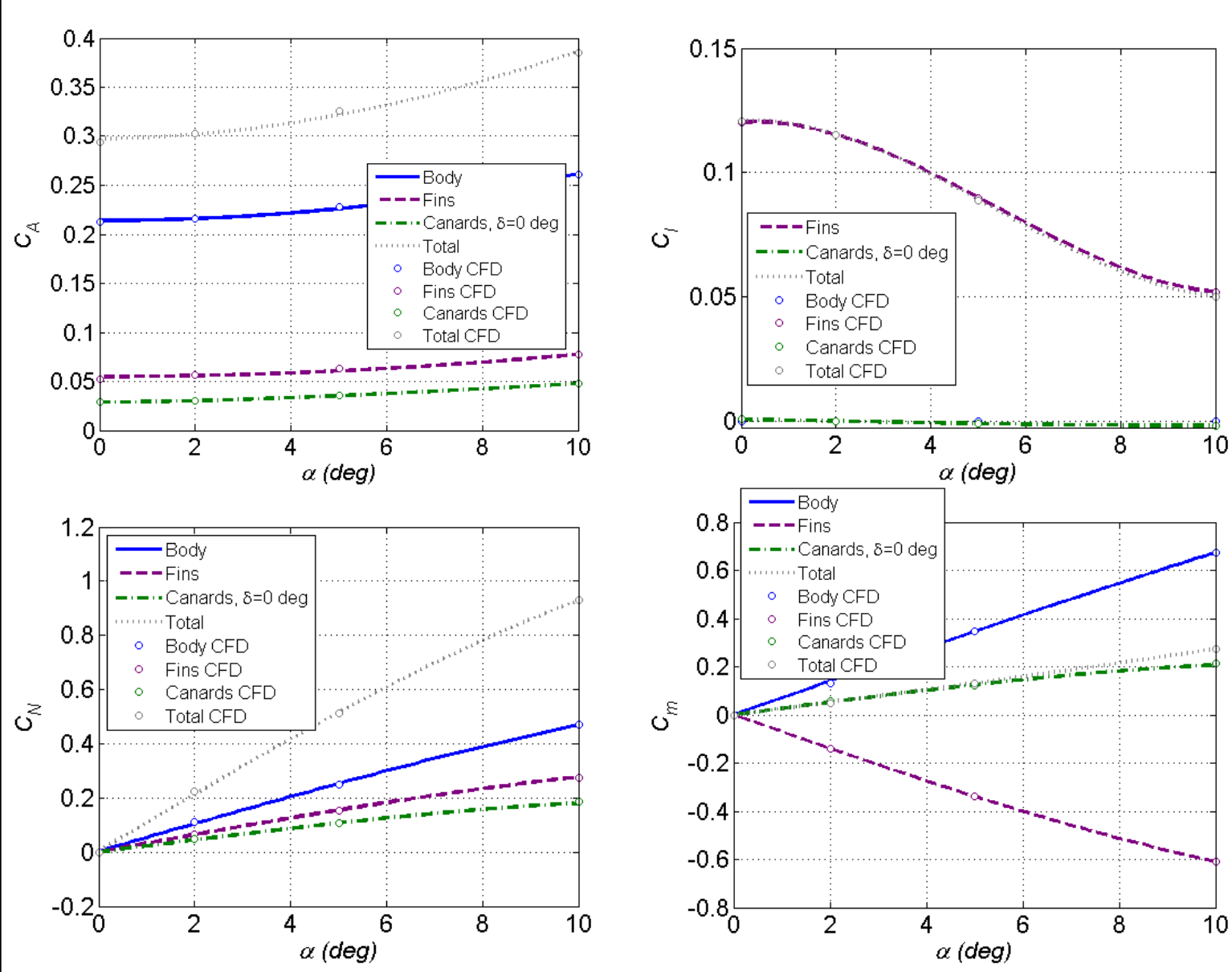

Fig. B-18 Component fit build up comparison to total CFD coefficients for Configuration 1b, Mach 0.8 
INTENTIONALLY LEFT BLANK. 


\section{List of Symbols, Abbreviations, and Acronyms}

\begin{tabular}{|c|c|}
\hline$\dot{w}_{M}$ & measured pitch acceleration $\left(\mathrm{m} / \mathrm{s}^{2}\right)$ \\
\hline$\dot{w}_{r}$ & reference pitch acceleration $\left(\mathrm{m} / \mathrm{s}^{2}\right)$ \\
\hline$C_{l_{p}}$ & roll damping moment coefficient \\
\hline$C_{A}$ & axial force coefficient \\
\hline$C_{N}$ & normal force coefficient \\
\hline$C_{l}$ & axial moment (roll torque) coefficient \\
\hline$C_{m}$ & pitching moment coefficient \\
\hline$q_{M}$ & measured pitch rate $(\mathrm{m} / \mathrm{s})$ \\
\hline$q_{r}$ & reference pitch rate $(\mathrm{m} / \mathrm{s})$ \\
\hline$\dot{w}$ & actual pitch acceleration $\left(\mathrm{m} / \mathrm{s}^{2}\right)$ \\
\hline$x_{c_{r e f}}$ & $\begin{array}{l}\text { canard hinge location at which canard aerodynamic coefficients were determined } \\
\text { (cal.) }\end{array}$ \\
\hline$B$ & body \\
\hline$C$ & canard \\
\hline$C_{p}$ & pressure coefficient \\
\hline$D$ & reference diameter $(\mathrm{m})$ \\
\hline$d y$ & first cell spacing off wall (m) \\
\hline$F$ & fin \\
\hline$f_{c}$ & canard-scaling factor \\
\hline$f_{F}$ & fin-scaling factor \\
\hline$I_{x}$ & axial moment of inertia $\left(\mathrm{kg}-\mathrm{m}^{2}\right)$ \\
\hline$I_{y}$ & transverse moment of inertia $\left(\mathrm{kg}-\mathrm{m}^{2}\right)$ \\
\hline $\mathrm{k}$ & turbulent kinetic energy \\
\hline$L / D$ & lift-to-drag \\
\hline$M$ & Mach number \\
\hline
\end{tabular}




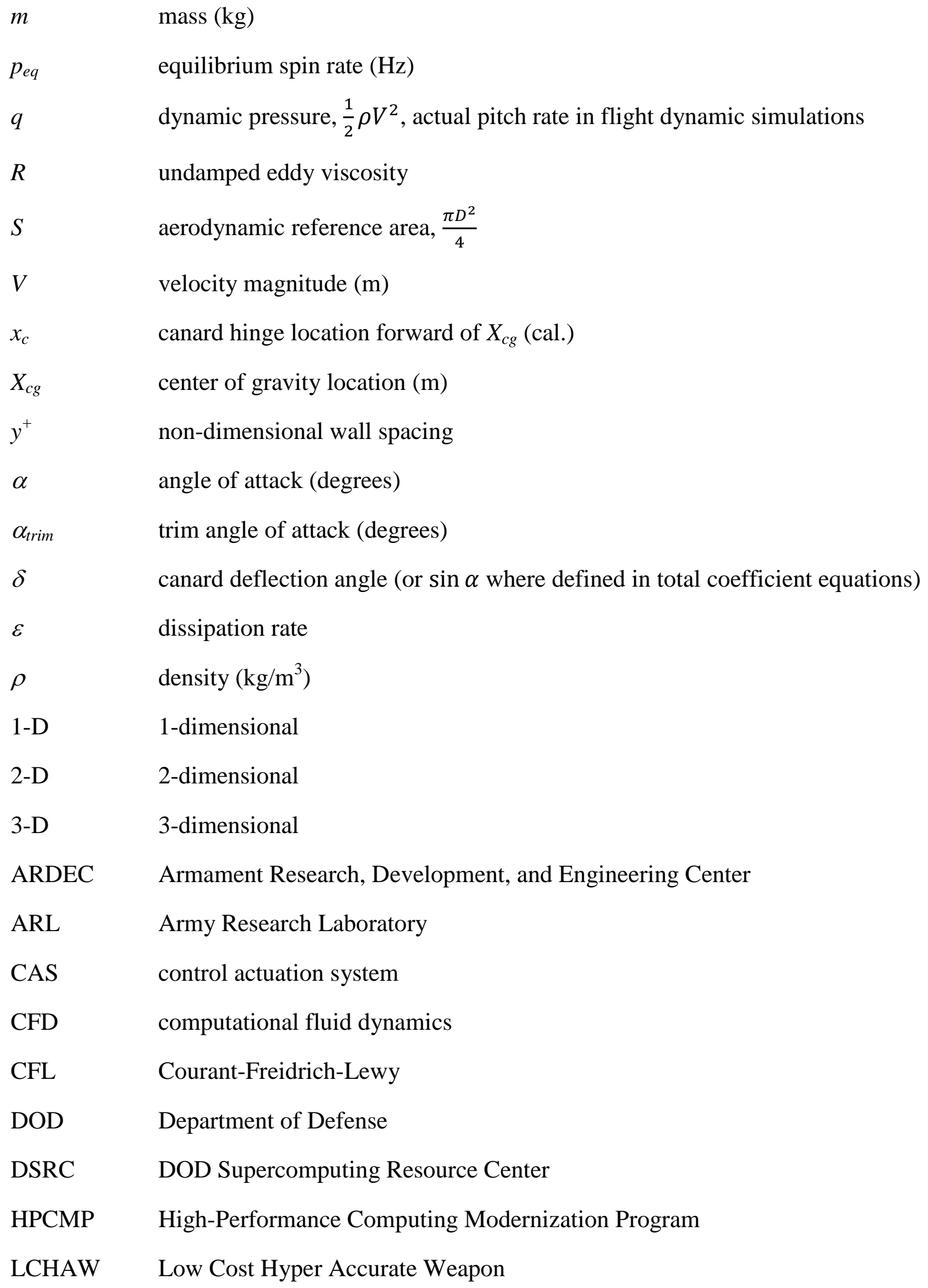


MPI Message Passing Interface

PVI Parallel Virtual Machine

RANS Reynolds-averaged Navier-Stokes

VAPP Very Affordable Precision Projectile 


\begin{tabular}{|c|c|c|c|}
\hline 1 & DEFENSE TECHNICAL & & W TOLEDO \\
\hline (PDF) & INFORMATION CTR & & J M TRAVAILLE \\
\hline & DTIC OCA & & $\begin{array}{l}\text { R S TROHANOWSKY } \\
\text { E B VAZQUEZ }\end{array}$ \\
\hline 2 & DIRECTOR & & L T VO \\
\hline$(\mathrm{PDF})$ & US ARMY RESEARCH LAB & & \\
\hline & RDRL CIO LL & 4 & PM CAS \\
\hline & IMAL HRA MAIL \& RECORDS MGMT & $(\mathrm{PDF})$ & $\begin{array}{l}\text { M BURKE } \\
\text { R KIEBLER }\end{array}$ \\
\hline 1 & GOVT PRINTG OFC & & P MANZ \\
\hline (PDF) & A MALHOTRA & & G SCHWARTZ \\
\hline 8 & AMRDEC & 3 & PM MAS \\
\hline (PDF) & L AUMAN & (PDF) & J FOULTZ \\
\hline & S DUNBAR & & C GRASSANO \\
\hline & B GRANTHAM & & D RIGOGLIOSO \\
\hline & K KENNEDY & & \\
\hline & J DOYLE & 2 & NWSCDD \\
\hline & J KEENAN & (PDF) & L STEELMAN \\
\hline & M MCDANIEL & & S KOSKI \\
\hline & C ROSEMAN & & \\
\hline & & 40 & DIR USARL \\
\hline 38 & ARDEC & (PDF) & RDRL WM \\
\hline (PDF) & D E CARLUCCI & & P BAKER \\
\hline & J H CHOI & & R EHLERS \\
\hline & S K CHUNG & & RDRL WML \\
\hline & D CIMORELLI & & P PEREGINO \\
\hline & B J DEFRANCO & & M ZOLTOWSKI \\
\hline & D DEMELLA & & RDRL WML A \\
\hline & M S DUCA & & W OBERLE \\
\hline & P C FERLAZZO & & RDRL WML B \\
\hline & J A FONNER & & N TRIVEDI \\
\hline & R M FULLERTON & & RDRL WML C \\
\hline & R P GORMAN & & S AUBERT \\
\hline & R F GRANITZKI & & RDRL WML D \\
\hline & J C GRAU & & R BEYER \\
\hline & N J GRAY & & RDRL WML E \\
\hline & M E HOHIL & & V BHAGWANDIN \\
\hline & M S HOLLIS & & I CELMINS \\
\hline & R T HOOKE & & J DESPIRITO \\
\hline & W H KOENIG & & L FAIRFAX \\
\hline & A LICHTENBERG-SCANLAN & & F FRESCONI \\
\hline & J A LONGCORE & & J GARNER \\
\hline & M F LUCIANO & & B GUIDOS JR \\
\hline & G MALEJKO & & K HEAVEY \\
\hline & M G MARSH & & G OBERLIN \\
\hline & G T MINER & & J SAHU \\
\hline & C MOEHRINGER & & P WEINACHT \\
\hline & J F MURNANE & & RDRL WML F \\
\hline & D W PANHORST & & B ALLIK \\
\hline & D D PASCUA & & T BROWN \\
\hline & T G RECCHIA & & E BUKOWSKI \\
\hline & J M ROMANO & & J CONDON \\
\hline & A F SANCHEZ & & M DON \\
\hline & K R SANTANGELO & & J HALLAMEYER \\
\hline & C G STOUT & & M HAMAOUI \\
\hline
\end{tabular}


P HUFNAL

M ILG

B KLINE

J MALEY

C MILLER

B NELSON

B TOPPER

B DAVIS

RDRL WML G

M CHEN

M MINNICINO

J SOUTH

RDRL WML H

J NEWILL

RDRL WMM

J ZABINSKI

RDRL WMP

D LYON 
INTENTIONALLY LEFT BLANK. 